Florida International University FIU Digital Commons

$12-12-1986$

\title{
Study of the effectiveness of cost-estimation models and complexity metrics on small projects
}

Lein-Lein Chen

Florida International University

DOI: $10.25148 /$ etd.FI14060166

Follow this and additional works at: https://digitalcommons.fiu.edu/etd

Part of the Computer Sciences Commons

\section{Recommended Citation}

Chen, Lein-Lein, "Study of the effectiveness of cost-estimation models and complexity metrics on small projects" (1986). FIU Electronic Theses and Dissertations. 2134.

https://digitalcommons.fiu.edu/etd/2134 


\begin{abstract}
STUDY OF THE EFFECTIVENESS OF COST ESTIMATION MODELS

AND COMPLEXITY METRICS ON SMALL PROJECTS

by

Lein-Lein Chen
\end{abstract}

Software cost overruns and time delay are common occurrences in the software development process. To reduce the occurrences of these problems, software cost estimation models and software complexity metrics measurements are two popular approaches used by the industry.

Most of the related studies are conducted for large scale software projects. In this thesis, we have investigated the effectiveness of three popular cost estimation models and program complexity metrics in so far as their applicability to small scale projects is concerned. Experiments conducted on the programs collected from FIU and NCR corporation indicate that none of the cost estimation models precisely estimates the actual development effort. However, the regression results indicate that the actual development effort is some function of the model variables. In addition, it also showed that the complexity metrics are useful measurements in predicting the actual development effort. Additional results related to lines of code metric are also discussed. 
To Professor Jainendra Navlakha, John Comfort, Robert Fisher, Samuel Shapiro,

This Thesis, having been approved in respect to form and mechanical execution, is referred to you for judgment upon its substantial merit.

Dean Professor James Mau

College of Arts and Sciences

The thesis of Lein-Lein Chen is approved.

\author{
Professor John Comfort \\ Professor Robert Fisher \\ Professor Samuel Shapiro
}

Major Professor Jainendra Navlakha

Date of Examination:

December 12,1986 
STUDY OF THE EFFECTIVENESS OF COST-ESTIMATION MODELS AND COMPLEXITY METRICS ON SMALL PROJECTS

$$
\text { by }
$$

Lein-Lein chen

\begin{abstract}
A thesis submitted in partial fulfillment of the requirements for the degree of
\end{abstract}

MASTER OF SCIENCE

in

COMPUTER SCIENCE

at

FLORIDA INTERNATIONAL UNIVERSITY

November 1986 


\section{ACKNOWLEDGEMENTS}

I wish to express my sincere gratitude to Dr. Jainendra Navlakha for his guidance throughout this work. In particular, his suggestions and reference aids were invaluable. I would also like to thank Dr. Carlos Brain, Dr. Paulette Johnson and especially Dr. Samuel Shapiro for sharing their knowledge of statistics and methods. My appreciation must also be extended to Dr. Wesley Mackey for familiarizing and authorizing my use of laser printer which made this printing possible.

In addition, my appreciation is also extended to all my friends and the faculty of computer science for their help, patience and data. And lastly, I am deeply indebted to Li Qiang for use of his metrics analyzer program which made part of the data collecting work much easier. 


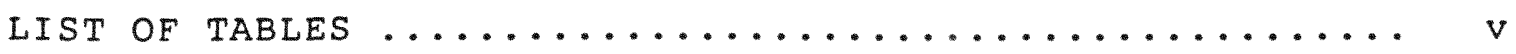

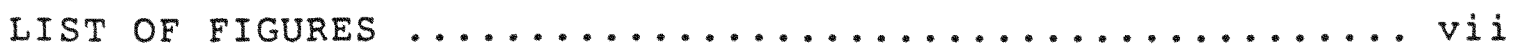

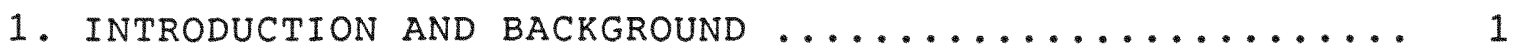

2. DESCRIPTION OF COST ESTIMATION MODELS ............. 7

IBM Walston-Felix Model $1977 \ldots \ldots \ldots \ldots \ldots$

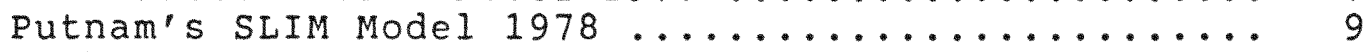

Boehm's Constructive Cost Model (Cocomo) $1981 \ldots . . .12$

3. REVIEW OF SOFTWARE COMPLEXITY METRICS ............ 16

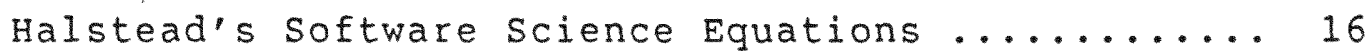

McCabe's Cyclomatic Complexity Measure ........... 18

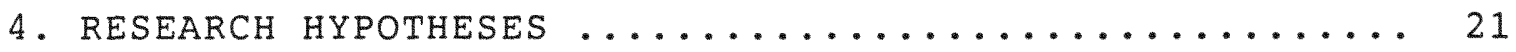

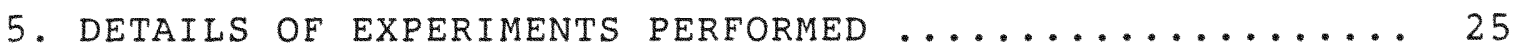

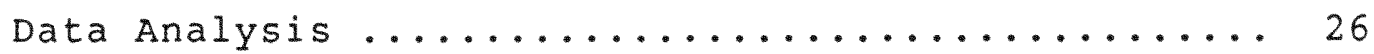

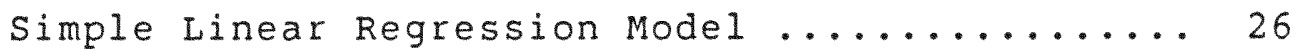

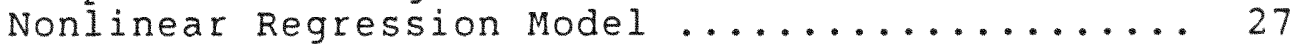

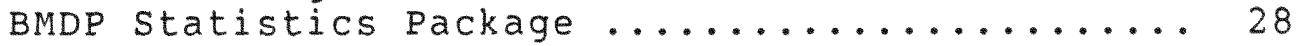

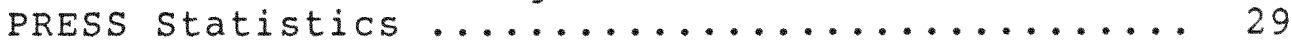

Proposed statistical Analyses ................ 30

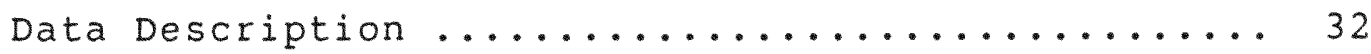

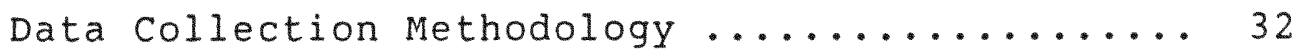

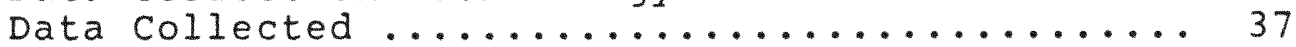

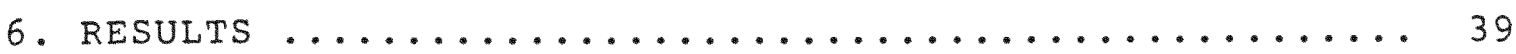

The Effectiveness of Cost Estimation Models-IBM, SLIM, and COCOMO on predicting Development Effort for Small scale projects.......

The Relationship between Actual Development Effort and McCabe's Cyclomatic Complexity ............. 44

The Relationship between Actual Development Effort

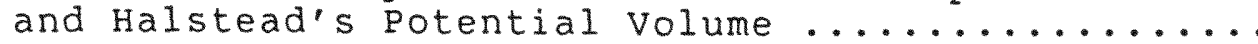


The Relationship between the Number of Instructions (DSI), the Total Number of Distinct symbols (n), the Program volume (V) and the Cyclomatic Complexity $(\mathrm{V}(\mathrm{G})) \ldots \ldots \ldots \ldots \ldots \ldots$

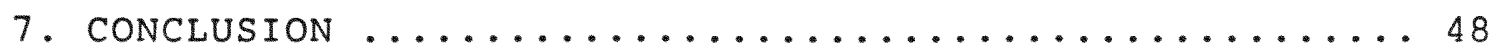
APPENDICES

A. Sample PASCAL program ........................ 99

B. Output of the Metrics Analyzer Program ...........101

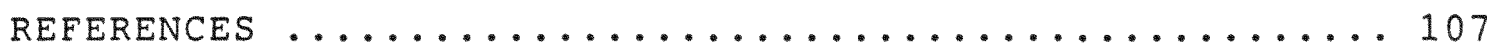




\section{LIST OF TABLES}

Table 1. IBM 29 Variables that correlate Significantly with programming

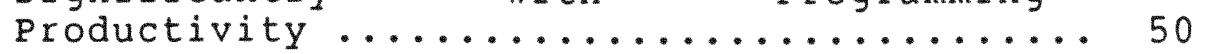

Table 2. Range of Technology constant of a very Simple software Cost Estimation system

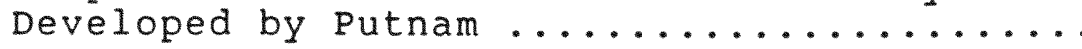

Table 3. Fifteen Effort Multipliers of Boehm's Intermediate cocomo model ............ 54

Table 4. Ratings of Cocomo Development Effort

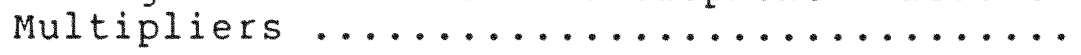

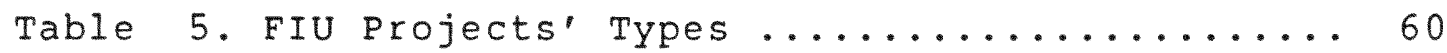

Table 6. Complexity Metrics Parameters of FIU Projects-54 PASCAL Programs ............ 61

Table 7. Calculations of McCabe's Cyclomatic Complexity for FIU 54 Programs ...........

Table 8. Complexity Metrics and Actual Development

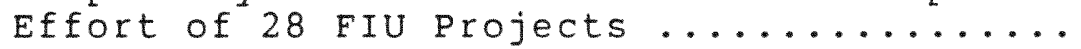

Table 9. Calculated Technology Constant and Technology Constant Derived by Putnam for

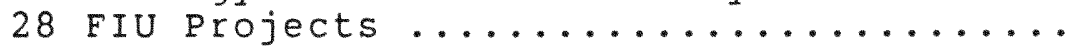

Table 10. Ratings of the Organic Mode of Boehm's Intermediate cocomo Model for 28 FIU

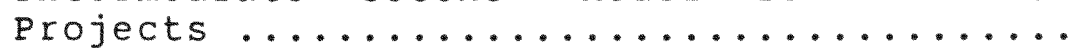

Table 11. Ratings of six Effort Multipliers of Adjusted cocomo Model for 28 FIU projects .. 70

Table 12. Complexity Metrics of 324 NCR Programs .... 72

Table 13. Actual Development Effort and Calculated 
Model Development Efforts for 28 FIU

Projects ......................... 79

Table 14. Relative Errors of Models' Development Efforts of FIU Projects ............. 80

Table 15. Relative Errors of Models' Development Efforts of FIU Projects with Program Lengths over 1000 Lines .............. 82

Table 16. Linear Regression Results of FIU Projects.. 84

Table 17. Nonlinear Regression Results of FIU

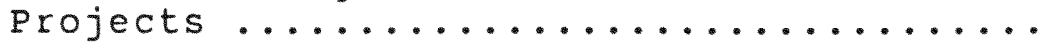

Table 18. Linear Regression Results of 324 NCR Programs .................... 88

Table 19. Nonlinear Regression Results of 324 NCR

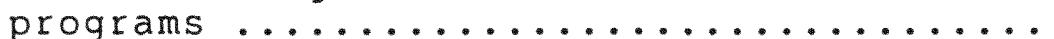

Table 20. Values of Correlation and Residual Mean Square between the Actual and Predicted Development Efforts of All Models on 28

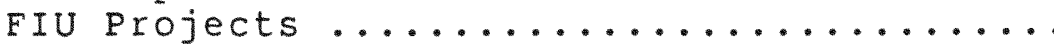

Table 21. Values of Correlation and Residual Mean Square between DSI and $n$, DSI and $V$, DSI and $V(G)$ of FIU and NCR Programs....

Table 22. The Instruction Density (DSI/V(G)) of 54

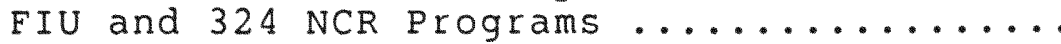




\section{LIST OF FIGURES}

Figure 1. Rayleigh Distributions with Various values of variance ............... 90

Figure 2. Pattern of Life-Cycle Effort Required to complete a Large scale software

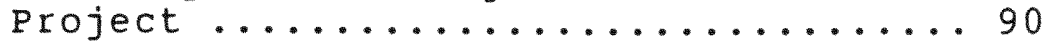

Figure 3. Manpower Pattern of Software Development for Large software systems Interpreted by Putnam ............. 91

Figure 4. Software Life Cycle with stages of Software Development Interpreted by

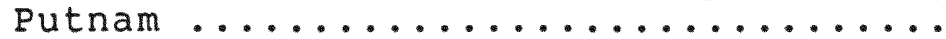

Figure 5. I/O Characteristics of the Metrics

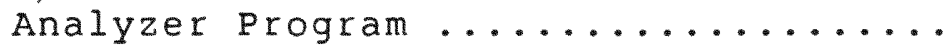

Figure 6. Regression Line with 95\% Confidence Interval for ACTEFF on IBMEFF .......

Figure 7. Regression Line with $95 \%$ Confidence Interval for ACTEFF on SLIMEFF ......

Figure 8. Regression Line with 95\% Confidence Interval for ACTEFF on COCOMOEFF .....

Figure 9. Regression Line with 95\% Confidence Interval for ACTEFF on ADJUSTEFF .... 95

Figure 10. Regression Line with 95\% Confidence Interval for ACTEFF on HLSTDEFF ..... 96

Figure 11. Regression Line with 95\% Confidence Interval for ACTEFF on $V(G) \ldots . . .$.

Figure 12. Regression Line with 95\% Confidence Interval for ACTEEF on $V^{*} \ldots \ldots \ldots . .$. 


\section{INTRODUCTION AND BACKGROUND}

Cost overruns and time delays in software development have been two major problems in the computer industry for a period of time. It is estimated that the total expenditures on all aspects of computing in the United state in 1980 was approximately 5 percent of the Gross National Product (GNP), or about $\$ 130$ billion. It is further estimated that computing revenues will be 12.5 percent of the GNP by 1990 [7]. Thus the solution to the problem of cost overruns will provide tremendous financial benefits. In large-scale software development projects it is quite common for the costs to be double or triple the original estimate. Associated with these increasing software costs is the problem of time delays where up to 100 percent slippages have been quite common in the software development process [22]. This problem of late delivery can lead to a project's failure, and usually will increase the software development costs. On the other hand, as the software development costs have been increasing, the costs for hardware have been decreasing. In 1960, the ratio was approximately 80 percent hardware cost to 20 percent software cost for a system. BY 1980, the ratio was reversed: approximately 20 percent hardware cost to 80 percent software cost, and by 1990 , software costs will increase even more and will account for 90 percent of the amount spent on a computing system [7]. The rate of growth for the cost of software is greater than that of the United States economy in general [13]. The high 
cost of software necessitates development of new techniques for quality software development and efficient cost control. During the past decade, many techniques have been proposed and/or developed in all areas of software engineering. One such development is the use of models and metrics based on the historical data and experience to quantitatively estimate the cost and provide better managerial control of projects. Two areas for research and development to address these issues have evolved. They are the development of cost estimation models which predict the costs of software, and the measurements of the attributes of software which enhance our understanding of the software development process.

A cost model is a formula or a set of formulas used to predict the costs likely to be incurred in a project [6]. Most of the cost models are similar in the sense that they are derived from the historical data base of an organization and use the number of lines of code (the number of source lines including or excluding comments) as the major factor to determine the cost of software. In addition, it is found that the environmental factors such as the ability of the personnel involved in developing the projects, techniques used (modern programming practices), target machines, languages, and application's complexity etc. also influence the software development cost. Some models incorporate such attibutes in the cost function as effort multipliers (also called cost drivers). Theoretically, there are many parameters that can affect the software costs. The general approach to cost modeling is to list all possible factors 
and try to find a function by statistical analysis that performs well on the historical data. The model is built by selecting a set of variables which are significantly related to the variations of some specific attributes of the models such as productivity, effort etc. In addition to the cost of software which is determined by the amount of effort, other estimates such as software development time, manpower, risks, and trade-off etc. also can be computed by some of the cost estimation models. Cost models are very popular in industry, and they are being used by many organizations to predict the software development costs and schedules.

Software metrics are another important tool which help in one's understanding of the software development process. It is the area of software engineering that deals with techniques used to measure various properties of a software product. They can be used to measure many properties and attributes of software including its quality, complexity, productivity, reliability, maintainability, correctness, portability, and development effort. In addition, such measurements also can be used as a tool to manage resources and evaluate the quality of a design so that changes and improvements can be made during the software development process. Their importance in the software industry is quite evident. The general process to develop a software metric is as follows:

1. make some observations regarding an attribute of software;

2. hypothesize a set of principles to explain the 
observations and intuitively define formulas for the metric;

3. perform experiments in a controlled environment to verify the accuracy of the metric; and

4. accept the metric as defined or make some adjustments to the hypotheses and formulas and loop back to step 3 .

clearly the development of software metrics is a trial and error process. Its accuracy depends on one's ability to perform good experiments in a controlled environment. Among all metrics, complexity metrics which measure the complexity of a program have gained maximum attention. Software complexity is the measure of the resources another system will expend while interacting with the software. It is also defined as the difficulty of manipulating software. One approach to define complexity metrics is based on the structure (or style) of the source code itself. In our research we concentrate on this type of complexity metrics, called program complexity metrics.

Almost all cost models $[4,10,12,21,23,26,28]$ developed to date have been used for predicting the cost of software development of large projects. Very few experiments have been performed to test the applicability of these models for small projects. However there have been some studies [9] that have successfully applied complexity metrics to small projects. Since complexity metrics may measure the difficulty of the program, and since the actual development effort is proportional to the difficulty, hence the development effort should be related to these metrics. 
Consequently, variations of the software costs from project to project should be explainable by the difference in program complexity as measured by the metrics. Present measurements of estimating the cost of software product are inadequate. Therefore it is desirable to search for new relationships which can be applied to the factors and which will result in more accurate predictions and hence better decisions may be made for controlling the cost and quality of the software system. This will improve the probability of success. We therefore, decided to conduct a study focusing on the following issues pertaining to the development of small projects:

a. The effectiveness of software cost estimation modelsIBM, SLIM and COCOMO, and the relationship between the actual development effort and the predicted development effort computed by these models.

b. The relationship between complexity metrics and the actual development effort.

c. The relationship among the complexity metrics themselves. This report is organized as follows. In section 2 , three popular cost estimation models- IBM, SLIM and COCOMO which are relevant to our study are introduced. Section 3 reviews the software complexity metrics; two techniquesHalstead's software science equations and McCabe's cyclomatic complexity metrics are described. Section 4 proposes four research hypotheses and anticipated results. The details of the experiments performed investigating each hypothesis, the source of the data, descriptions of the 
statistical analyses, the variables used in statistical analyses, the data collection methodologies and tabular data appear in section 5. Next, the experimental results are discussed for each research hypothesis. Finally, section 7 contains the conclusions and some further inferences. 


\section{DESCRIPTION OF COST ESTIMATION MODELS}

There exist many cost models in use in industry today such as SDC, DOTY, RCA PRICE, IBM Walston-Felix, Putnam's SLIM, Gruman's SOFCOST, Boehm's COCOMO, and Jensen's SEER, etc. $[4,10,12,13,22,23,26,28$,$] . This study will be limited$ to three of these. They were selected because the details are available freely in the literature (many are classified), they are popular in the industry and are respresentatives of most of the existing models. The three models are IBM Walston-Felix, Putnam's SLIM, and Boehm's сосомо.

\subsection{IBM Walston-Felix Model 1977 [26]}

The basic relationship between the number of lines of code and development effort was derived by walston and Felix and is based on the study of sixty projects at IBM. The least squares fit to this data yields the result

$$
E=5.2 *(L) 0.91
$$

where, $E$ is the total effort in man months, and $L$ is the size in thousands of lines of delivered source code including comments. In addition the authors also developed a measure of productivity. From the data base, they found twenty-nine variables (see Table 1) which showed significantly high correlations with productivity in their environment and were used in estimating the productivity index. The equation is defined as

$$
I=\sum_{i=1}^{n} w_{i} *\left(x_{i}\right)
$$

where 
I: productivity index for a project

$\mathrm{W}_{\mathrm{i}}$ : a factor weight based upon the productivity change for factor i

$x_{i}$ : equals $+1,0$, or -1 depending on whether the factor indicates increase, nominal, or decreased productivity other formulas given in this model include:

a) The relationship between documentation and delivered source lines is defined as

$$
D=49 *(L)^{1.01}
$$

where, $D$ is the number of pages of documentation, and $L$ is source code in thousands of lines.

b) The relationship between project duration and delivered source lines is given by

$$
M=4.1 *(L)^{0.36}
$$

where, $M$ is the duration in months, and $L$ is source code in thousands of lines.

c) The relationship between project duration and effort is as follows:

$$
M=2.47 *(E)^{0.35}
$$

where, $M$ is the duration in months, and $E$ is total effort in man-months.

d) The relationship between average staff size and effort is as follows:

$$
S=0.54 *(E)^{0.6}
$$

where, $S$ is the average number of people on staff, and $E$ is the total effort in man-months.

e) The relationship between computer cost and delivered source lines is given by 


$$
C=1.84 *(L)^{0.96}
$$

where, $C$ is the computer cost in thousands of dollars and $L$ is soruce code in thousands of lines. The constants in the above equations are derived from the historical data of IBM.

\subsection{Putnam's SLIM Model 1978 [19-22]}

This model is based on the empirical evidence that the pattern of life-cycle effort (in terms of man-year or man-month or man-hour) required to complete a large scale software project follows a Rayleigh distribution [25] (see Figure 1). Since this pattern was first shown by Norden (see Figure 2), it is also called the Rayleigh-Norden distribution [19]. Putnam empirically found that many medium to large scale software projects from different application areas exhibited the same life cycle pattern- a rise in manpower, a peaking and a tailing off (see Figure 3). For large systems, development cost is about 0.4 of the whole life cycle cost. The over all life-cycle curve of software development (see Figure 4) can be divided into stages such as systems definition, functional specification, software design, etc. Each stage provides better estimation than the previous one because more precise information about the size of the software system is available. The fundamental relationship among the source statements, the effort, the development time, and the state of the technology being applied to the project can be illustrated by the software equation

$s=\left(C_{k}\right) *(K)^{1 / 3} *\left(T_{d}\right)^{4 / 3}$ 
where

$K:$ the total life-cycle effort in man-years;

$T_{d}$ : the development time in years (time of peak manpower);

$\mathrm{C}_{\mathrm{k}}$ : the state of technology constant which is calibrated from the organization's historical data. Its range for simple software cost estimating system is presented in Table 2 .

S: the number of end product's delivered source lines of code.

Putnam used the technology constant to describe the environment under which the software was developed. This constant quantifies such factors as

. complexity of the system to be developed,

-development machine throughput capacity,

. software engineering tools,

- user interface (batch or interactive),

-target machine,

. development computer availability,

.discipline(modern programming practices),

. I anguage, and

. human skills.

The total life cycle effort is given as

$$
K=\left(\left(s / C_{k}\right)^{3}\right) /\left(T_{d}\right)^{4}
$$

and the development effort is 40 percent of the total life-cycle effort. i.e.

$$
E=0.4 *(K)
$$

That means, if everything is the same, then a large value of 
technology constant will imply less development effort. From empirical data, Putnam also found that $k /\left(\mathrm{T}_{\mathrm{d}}\right)^{2}$ appeared to correspond to the difficulty of the system. Those systems which had been regarded as easy had small values of $\mathrm{K} /\left(\mathrm{T}_{\mathrm{d}}\right)^{2}$, and those which had been regarded as hard had large values. He also found that the trade-off of effort and time can be explained by the software equation $K=$ constant $/\left(T_{d}\right)^{4}$

From the relationship one sees that small changes in development time result in very large changes in effort. Putnam also mentioned that the development schedule can not be arbitrarily compressed by adding more resources in the system. The PERT sizing method is applied to estimate the size (in terms of number of source lines of code) of the software product at the beginning of the software development phase, which is then used with other parameters to determine the development effort as described before. A Monte Carlo simulation method is used to generate the milestones of the project in terms of fraction of total development time. Empirically studies give the following results:

$$
\left(t / T_{d}\right)
$$

Critical Design Review .43

systems Integration Test

Prototype Test .80

start Installation 
The same simulation method is also used to determine the risk (expressed in terms of probability) that a software product can be done within a specific value of cost, time and effort. For example, the probability of developing a specific project with five million dollars was $90 \%$ which is substantially higher than that with four million dollars $(50 \%)[22]$.

Generally speaking, there exist many constraints such as contract delivery time, maximum peak man power available etc. that are imposed on the software product development. In the SLIM model, these constraint inequalities are expressed as functions of $\mathrm{k}$ and $\mathrm{T}_{\mathrm{d}}$. As all the equations are exponential in nature, they are linearized by taking logarithms. Linear programming techniques are used to determine the feasibility region from where management can perform cost/time tradeoffs for any project.

2.3 Boehm's Constructive Cost model (Cocomo) $1981[2,3,4]$ COCOMO was developed at TRW by Boehm. It represents a hierarchy of three models, Basic, Intermediate, and Detailed, which increase in precision. The model uses the number of team members, the project type and some other project and development environment characteristics as the basic variables. Each model may be applied in three different modes-organic, Semidetached, and Embedded. The сосомо estimating equations were obtained by analyzing a sample of sixty-three software projects in the data base. The Basic model uses only the number of instructions to 
predict the development effort and hence its accuracy is only good enough for use in the early stages of a project. The nominal effort equations used in the Basic cocomo are listed below:

Organic mode: $\quad \operatorname{Effort}(M M)=2.4 *\left((\operatorname{KDSI})^{1.05}\right)$

Semidetached mode: Effort $(M M)=3.0 *\left((\operatorname{KDSI})^{1.12}\right)$

Embedded mode: $\quad$ Effort $(M M)=3.6 *\left((\operatorname{KDSI})^{1.20}\right)$

Where MM: man-months,

KDSI: source instruction in thousands of lines.

The Intermediate cocomo model considers a set of fifteen variables which are called cost drivers or effort multipliers to explain much of the variations in software costs for different projects. These additional factors are grouped into the following four categories:

- Product attributes

RELY Required Software Reliability

DATA Data Base Size

CPLX Product Complexity

- Computer Attributes

TIME Execution Time Constraint

STOR Main Storage Constraint

VIRT Virtual Machine Volatility

TURN Computer Turnaround Time

- Personnel Attributes

ACAP Analyst Capability

AEXP Applications Experience

PCAP Programmer Capability

VEXP Virtual Machine Experience 
LEXP Programming Language Experience

.Project Attributes

MODP Modern Programming Practices

TOOL Use of Software Tools

SCED Required Development Schedule

Each of these cost drivers (effort multipliers) is defined by a set of weights which depend on the particular project (see Table 3 and Table 4 ).

The general concepts of the Detailed cocomo model are similar to the Intermediate cocomo except that it decomposes the software product into module, subsystem, and system levels and uses phase sensitive effort multipliers for each cost driver attribute. These four phases are: Product Design (PD), Detail Design (DD), Code \& Unit Test (CUT) and Integration \& Test (IT). According to Boehm, some factors affect some phases much more than others. For example, projects with very high reliability requirements or hardware constraints will require more of an effort to integrate and test. Hence, for each of the effort multipliers, there is a weight corresponding to each phase, so the effort can be calculated by phase. The three level hierarchical decomposition of a software product from bottom to top is described below.

. Module level (lowest level)

It is described by the number of source instructions in the module, and by those cost drivers which tend to vary at the lowest level such as the module's complexity, the module programmers' capability and experience with the 
language being used.

. Subsystem level

It is described by the remainder of the cost drivers such as time constraint, analyst capability, tools, schedule etc. which tend to vary from subsystem to subsystem. . System level (top level)

It is a collection of all the subsystems. The nominal effort equations of all modes listed below are used in both Intermediate and Detailed cocomo models. Organic mode: $\quad$ Effort $(M M)=3.2 *\left((\text { KDSI })^{1.05}\right)$ Semidetached mode: $\operatorname{Effort}(M M)=3.0 *\left((\operatorname{KDSI})^{1.12}\right)$ Embedded mode: $\quad$ Effort $(M M)=2.8 *\left((\text { KDSI })^{1.20}\right.$, where MM: man-months,

KDSI: source instruction in thousands of lines. The main contribution that Detailed Cocomo provides beyond the Basic and Intermediate versions is a better basis for detailed project personnel planning with respect to the level of staff required to complete each development phase. In addition to estimating software cost from scratch, cocomo also has formulas for caculating the effort for code adopted from the existing software. Other estimates such as development schedule and maintenance costs are also given.

All the above cost models were developed for estimating the cost of large size projects because it is the costliest component in the system. We now investigate their usefulness for small size projects. 


\section{REVIEW OF SOFTWARE COMPLEXITY METRICS}

In the period prior to the mid 1970's, the only software complexity metric in use was the number of source lines of code. A system with more source lines was assumed to be more complex than another with less source lines. Starting in the mid $1970^{\prime} \mathrm{s}$, many other complexity metrics were developed, some were based on detailed and particular constructs of source code while the others were based on design structure chart and information flow in a system. The latter types by Yin \& Winchester [29] and Henry \& Kafura [11] are system complexty metrics and will not be considered here. This study will be limited to the metrics that depend on the source code or some particular features of the source code. Halstead [9] considered that each symbol of the code contributed towards software complexity, McCabe [14] assumed that only control flow transfers contributed to complexity while Albrecht [1] hypothesized that only I/O behavior and requirements determined the complexity of a software system. We describe the software complexity metrics of Halstead and McCabe which are relevant to our work.

\subsection{Halstead's Software Science Equations [9]}

Halstead considered each symbol of a program to be either an operator or an operand. The software science equations are based on the fundamental components of any program that are given below:

$\mathrm{n}_{1}$ : the number of distinct operators appearing in a program 
$\mathrm{n}_{2}$ : the number of distinct operands appearing in a program

$N_{1}$ : the total number of occurrences of the operators in a program

$\mathrm{N}_{2}$ : the total number of occurrences of the operands in a program

Based on these components, many other complexity metrics parameters are defined as follows:

$$
\begin{array}{ll}
\text { vocabulary } & \mathrm{n}=\mathrm{n}_{1}+\mathrm{n}_{2} \\
\text { Length } & \mathrm{N}=\mathrm{N}_{1}+\mathrm{N}_{2} \\
\text { volume } & \mathrm{V}=\mathrm{N} * \log _{2} \mathrm{n}
\end{array}
$$

where $\log _{2} n$ is the number of bits required to store each symbol of the program and hence volume gives the total number of bits needed to store the whole program. The volume will change depending upon the power of the programming language, so it is possible for different implementations of an algorithm to have different volumes. The minimum volume is called potential volume $\left(V^{*}\right)$ which is the property of the algorithm and is defined as

$$
\mathrm{v}^{*}=\left(\mathrm{n}_{1}{ }^{*}+\mathrm{n}_{2}{ }^{*}\right) * \log _{2}\left(\mathrm{n}_{1}{ }^{*}+\mathrm{n}_{2}{ }^{*}\right)
$$

where

$$
\begin{aligned}
\mathrm{n}_{1}{ }^{*} \text { : } & \text { represents the minimum number of distinct } \\
& \text { operators, }\left(\mathrm{n}_{1}{ }^{*}=2\right) ; \\
\mathrm{n}_{2}{ }^{*} \text { : } & \text { represents the minimum number of distinct } \\
& \text { operands to implement the algorithm. This number } \\
& \text { is very difficult to estimate. }
\end{aligned}
$$

Now, for a particular implementation of an algorithm, its level is determined by the ratio of potential volume of that 
algorithm and the actual volume of the program, i.e.

$$
\mathrm{L}=\mathrm{V}^{*} / \mathrm{V} ; \quad 0<\mathrm{L}<=1 \text {. }
$$

The difficulty of implementation is given by

$$
D=1 / L
$$

i.e. higher the level, less is the difficulty. As $\mathrm{V}^{*}$ is difficult to obtain (because $\mathrm{n}_{2}{ }^{*}$ can not be determined), the estimated level based on the use of operands and operators is calculated as

$$
L=\left(2 / \mathrm{n}_{1}\right) *\left(\mathrm{n}_{2} / \mathrm{N}_{2}\right) \text {. }
$$

The effort required to write the program (in terms of elementary mental discriminations or total number of "moments") is given by

$$
E=V / L, \text { or } E=V * D \text {. }
$$

The programming time $T$ (in seconds) is defined as

$$
T=E / S
$$

where $S$ is the stroud factor and has units of "moments" per second. It was found by stroud that a human brain is capable of performing between 5 to 20 elementary mental discriminations per second. Halstead used $S=18$ mental discriminations per second in his studies.

3.2 McCabe's Cyclomatic Complexity Measure [14]

McCabe's cyclomatic complexity measure depends on the control flow of that program, that is, its decision structure. His overall strategy is to compute the number of linearly independent paths in the directed graph obtained from the program flow graph. However, a program with a backward branch could have infinite number of paths, so his 
complexity measure is only defined in terms of the number of basic paths of a program. According to him the complexity measure, $V(G)$, is correlated closely with the amount of work required to test a program based on the number of basic paths in it. He applied the properties of graph theory to the characteristics of the program structure, where the program itself corresponds to a directed graph with a single entry and single exit. The blocks of sequential code in the program then correspond to each node in the graph, and the branches which change the program control flows correspond to the arcs in the graph. The cyclomatic complexity of a strongly connected graph $G$ is defined as

$$
V(G)=\text { Edges - Nodes }+2 \text { * Components }
$$

where the terms Edges and Nodes represent the total number of edges and nodes in the graph respectively, and the term Components is the total number of connected components in the graph. The following two examples given by McCabe illustrate this idea.

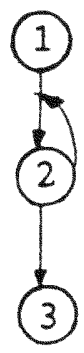

$$
\begin{aligned}
V(G) & =E-N+2 * C \\
& =3-3+2 * 1 \\
& =2
\end{aligned}
$$

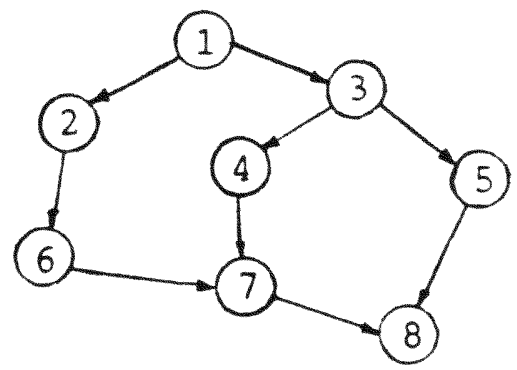

$$
\begin{aligned}
V(G) & =E-N+2 * C \\
& =9-8+2 * 1 \\
& =3
\end{aligned}
$$


It is also proved that in general the complexity of any program can be computed in terms of the number of simple predicates (decisions with single entry and single exit) in a program. Thus

$$
V(G)=\#+1
$$

where \# is the number of simple predicates in a program. Many variations to McCabe's complexity metrics exist. They all mainly differ from McCabe's metrics in the way in which conditions are counted. There is no evidence in the literature that they are better than McCabe's metrics and hence are not considered in this study. Mccabe also observed that $V(G)$ is a reasonable measure to compare and rank the complexity of various routines. An upper bound of 10 is recommened as the maximum complexity for the control graph of an individual routine. 


\section{RESEARCH HYPOTHESES}

As mentioned earlier, this work focuses on the following issues regarding small scale software projects.

a. The effectiveness of software cost estimation modelsIBM, SLIM and COCOMO, and the relationship between the actual development effort and the predicted development effort computed by these models.

b. The relationship between complexity metrics and actual development effort.

c. The relationship among the complexity metrics themselves. To test the relationships among cost model parameters, software complexity metrics and software development effort, several hypotheses are made. These are as follows:

H1. The formulas given by IBM, SLIM and CocoMo models can be used to predict the actual development effort for small projects.

Cost models like IBM, SLIM, and CoCOMO are popular models used in the industry for estimating the cost of large software projects. They have been shown to be very useful in resource management and cost control. However, no study has demonstrated that these models are also applicable to small projects. Since the basic formulas defined by these three models are all in terms of the program size, and small projects intuitively need less development effort, therefore we hypothesize that these models should also be usable for prediction of actual development effort for small scale software projects.

H2. McCabe's cyclomatic complexity is proportional to the 
amount of actual development effort.

According to Mccabe, the cyclomatic complexity of a program can be used to measure the difficulty of understanding and testing that program. Therefore, a program with more control paths than another will need more development effort for design, coding, debugging, and testing.

H3. Halstead's potential volume for an algorithm is proportional to the amount of actual effort required to implement it.

Halstead's potential volume measures the complexity of an algorithm in the sense that it is the minimum volume for an algorithm to be expressed in the most powerful language for that application. If potential volume increases, then the minimum volume of implementation of that algorithm increases which in turn means that the actual effort to implement it should also increase.

H4. The number of instructions is proportional to the total number of distinct symbols, the program volume, and the cyclomatic complexity of a program.

The number of source instructions has been commonly used as a measure of program size, and many predictions such as development effort and productivity are defined as a function of this measure. Since it is a very significant factor in all cost models and it is easy to compute, we investigated the relation of this attribute with other fundamental software complexity metrics.

various descriptive measures from the source program 
were obtained in order to test these hypotheses. The notations used for these measures are described below:

MEASURE

DSI

SLOC

n

N

V

$v^{*}$

$E$

$V(G)$

IBMEFE
ACTEFF DESCRIPTION

The number of source lines of instruction excluding comments (called delivered source instructions).

The number of source lines of code including comments.

Halstead's vocabulary size which is equal to the total number of distinct operators plus toal number of distinct operands.

Halstead's program length which represents the total occurrences of operators and operands appearing in a program.

Halstead's program volume.

Halstead's potential volume or minimum volume of an algorithm.

Halstead's program effort which represents the total number of elementary mental discriminations required for generating a program.

Mccabe's cyclomatic complexity of a program which may be expressed as the total number of predicates (conditions) plus one for a program.

$\operatorname{DSI} / \mathrm{V}(\mathrm{G})$ Which is the inverse of $V(G)$ density, we call it instruction density.

The actual effort to develop a software product. The development effort computed by IBM cost estimation model. 
24

SLIMEFF The development effort computed by Putnam's SLIM cost estimation model.

COCOMOEFF The development effort computed by the organic mode of Boehm's Intermediate cocomo cost estimation model.

$$
c_{k}
$$$$
\text { Technology constant of the SLIM cost estimation }
$$
model. 


\section{DETAILS OF EXPERIMENTS PERFORMED}

In order to statistically test the hypotheses described in the previous section, experimental data was collected from two different environments: Florida International University and the National Cash Register Corporation (denoted FIU and NCR hereafter). The FIU data, which was collected in spring 1986, consists of 54 PASCAL programs which were developed by students and professors in the Computer science program. The NCR data, which was collected in August 1985, was from 324 modules of a large project written in NCRL at the NCR corporation. These modules were included because each is a small program by itself and the group provides a good comparison between programs written in a university and an industrial environment. Two data sets were produced from the FIU group. One set contains PASCAL programs with varying complexities ranging from the introductary level programs to graduate level compiler projects (see Table 5) and some other projects of individual professors and students. Several projects were selected from the same course and thus these all had the same objective. The second group consisted of a subset of the first. This group consisted of 28 distinct programs for which the corresponding development effort was recorded. In some instances the programmer specified the actual development effort while for class projects the professors approximated the actual development effort. The detailed description of the analyses performed and data collection process is described below. 


\subsection{Data Analysis}

Regression analysis was used in this study for evaluating the previous stated hypotheses. In a regression analysis, the dependent (response) variable is expressed as a function of one or more independent (predictor) variables. The regression function estimates the average response for a given set of values of its independent variables. Thus it can be utilized to predict the average response for a given project.

Two types of regression models were used: simple linear regression and nonlinear regression. Computations were done using the software package BMDP (biomedical computer programs P-series) [5]. Another package SAS [24] was also used for plotting regression lines with $95 \%$ confidence intervals (see Figure 6-12). The adequacy of the simple linear regression model was measured by computing $\mathrm{R}^{2}$ using the PRESS [16] technique.

\subsubsection{Simple Linear Regression Model}

The simple linear regression model uses a single regressor $\mathrm{x}$, and is defined by

$$
Y=B_{0}+B_{1} X+e
$$

where the intercept $B_{0}$ and the slope $B_{1}$ are unknown constants and $e$ is a random error component. Since the parameters $B_{0}$ and $B_{1}$ are unknown and must be estimated using sample data, the method of Least squares is used. This technique finds those estimates of $B_{0}$ and $B_{1}$ which minimize the residual sum of squares (sum of squared 
differences between the observed and estimated values of $\mathrm{Y}$ ). The linear dependency between the $Y$ and $X$ variables is tested by use of an F-statistic which is the ratio of the regression mean square, MSR, and residual mean square, MSE. The coeffient of determination, $R^{2}$, is defined to be the square of the ordinary correlation coefficient, $r$. It is used to indicate the proportion of the total variability in the response variable $Y$ that can be accounted for by the predictor variable $\times[18]$.

5.1.2 Nonlinear Regression Model

A Nonlinear regression model is used when the relationship of the response to the independent variables can not be written in an expression which is linear in the parameters. The most general approach to the solution of a nonlinear problem is to attempt to get close enough to the best estimate, so that the nonlinear function may be approximated by the linear term in a Taylor series expansion. If this region can be entered, the problem has been linearized and the method of Least squares may be used [15]. The Least squares method estimates the parameters of a nonlinear function. For example, suppose the model is

$$
Y=A(B)^{X}+e \text {. }
$$

The model finds estimates of $A$ and $B$ by obtaining the values which minimize the sum of

$$
\left(Y-A(B)^{X}\right)^{2}
$$

taken over the sample values. Such method requires insertion of initial estimates of $A$ and $B$. Many methods 
have been developed in this field. One procedure which can be used with the above model is to obtain the initial estimates by using a log transformation to obtain a linear model and then using the least squares technique on the transformed model. This procedure was used in this analysis. The regression model produces the parameters of the nonlinear function and its residual mean square.

\subsubsection{BMDP statistics Package}

This package provides regression analysis and data ploting programs. For linear regression, program P9R (all possible subsets regression) was used. This program was used because of the extensive residual analysis it provides, such as the PRESS statistics and its data plotting routines. The nonlinear regression analysis was done using the program P3R. This program estimates the parameters of a nonlinear function using Least squares with a Gauss-Newton algorithm. The general formula which is selected by this program is defined below:

$$
Y=P 1(X)^{P 2}(\text { e })^{P 3(X)}+\ldots
$$

where

$Y$ : dependent variable

$\mathrm{x}$ : independent variable

P1,P2,P3: constants

In this application, the user has to specify the initial values of the constants P1 and P2; P3 and e are ignored by the system. The initial estimates of P1 and P2 were obtained by first using a log transformation as described 
above. Then the estimates of the intercept and slope were used as the initial values of $P 1$ and $P 2$ of the nonlinear function. The program $P 3 R$ reached its smallest residual mean square with a small number of iterations.

\subsubsection{PRESS statistics}

PRESS is one of the techniques used for evaluating regression models. The procedure works as follows: Select observation $Y_{i}$. Fit the regression model to the remaining n-1 observations and use the resulting equation to predict the withheld observation $\mathrm{Y}_{i}$, denoting this predicted value $\tilde{Y}_{(i)}$. Find the prediction error for point $i$ as

$$
e_{(i)}=Y_{i}-\tilde{Y}_{(i)} \text {. }
$$

The prediction error is often called the ith deleted residual. This procedure is repeated for each observation $i=1,2,3 \ldots, n$, producing a set of $n$ deleted residuals $e(1), e(2), \ldots, e(n)$. The PRESS statistic is defined as the sum of squares of the $n$ deleted residuals,

$$
\text { PRESS }=\sum_{i=1}^{n} e^{2}(i)=\sum_{i=1}^{n}\left(Y_{i}-\tilde{Y}_{(i)}\right)^{2}
$$

The approximate $R^{2}$ for prediction is then defined as

$$
\mathrm{R}_{\text {pred }}^{2}=1-\left(\text { PRESS } / \mathrm{S}_{Y Y}\right)
$$

where $S_{Y Y}$ is called the corrected sum of squares of the 
dependent variable. The BMDP statistic package, $P 9 R$, provides the information needed to calculate $\mathrm{R}^{2}$ pred; the average deleted residual and the standard deviation of dependent variable, $S_{Y}$. By multiplying average deleted residual by $n$ and $s_{Y}^{2}$ by $n-1$, the values for PRESS and $S_{Y Y}$ are obtained and the value of $R^{2}$ pred can then be obtained.

\subsubsection{Proposed Statistical Analyses.}

The following techniques were used to evaluate the various hypotheses. The observations were grouped into four different sets and analyzed as described below.

1. Analysis of the predicted development efforts produced by IBM,SLIM and COCOMO cost estimation models on small projects.

Two types of analyses were performed. The first compared the average relative error for each model. The average relative error was obtained by dividing the total relative errors by the number of projects. The relative error for each project was defined as follows:

Relative Error $=\frac{\text { ABS( Actual-Predicted ) }}{\text { Actual }}$

where

ABS: absolute value,

Actual: actual development effort,

Predicted: predicted development effort.

A second analysis compared the squared correlations, $R^{2}$, between the actual development effort and the predicted development effort for each model. The model error was 
estimated from the regression model using the residual sum of squares. Since all the model predictions are basically a function of the program size, and since the actual effort is proportional to the program size, the predictions should be close to the actual results.

2. Analysis of the relationship between actual development effort and McCabe's cyclomatic complexity.

A regression model was used to relate the actual development effort (dependent variable) to Mccabe's cyclomatic complexity (independent varible). There should be a strong positive relationship since the more complex a program the more development effort is needed.

3. Analysis of the relationship between Halstead's potential volume and actual development effort.

A Regression analysis was used to investigate the relationship of the actual development effort (dependent variable) and the potential volume (independent variable). since the actual development effort should be proportional to the complexity of the algorithms, there should be a strong positive relationship.

4. Analysis of the relationship of the number of instructions (DSI) with the total number of distinct symbols $(n)$, the program volume (V), and the cyclomatic complexity $(V(G))$.

It is intuitive that as the program size increases, so will the total number of distinct symbols, program volume, and the cyclomatic complexity, and vice versa. A regression analysis was performed on each pair using the number of 
instructions (DSI) as the dependent variable and $n, V$, and $V(G)$ as the independent variables respectively. A high correlation is expected for each pair.

\subsection{Data Description}

We have already identified the data required to perform the analysis described. The data collection methodologies and the description of the data collected are given below.

\subsubsection{Data Collection Methodology}

Programs were collected from FIU and NCR corporation. FIU programs included those from individual developers and students in selected classes. The data required from an individual's program was collected by interviewing the developer. Data on the class projects were obtained by consulting the instructor who approximated the development effort required. Since the instructor's approximates was used as the actual development effort of a completed project, only those projects that received ' $A$ ' or ' $A-$ ' grades were selected for the analysis. The data needed from the NCR source was available in a data base; however, this data did not include the actual development effort for each individual NCR module. Therefore these modules were excluded from the analysis relating to the model predictions.

5.2.1.1 Methodology Used for Collecting Complexity Metrics Parameters

The complexity metrics parameters of the FIU projects 
were measured using a 'Metrics Analyzer' program. This program was developed by Qiang and Navlakha [17] for analyzing the complexity of a standard PASCAL program and was modified for this project. This program takes a syntactically correct and executable PASCAL program as input and generates the values of Halstead's complexity metrics as well as the number of source lines as its output (see Figure 5). The output of the Metrics Analyzer program for a sample program (see Appendix A) that contains most features of PASCAL, is shown in Appendix B.

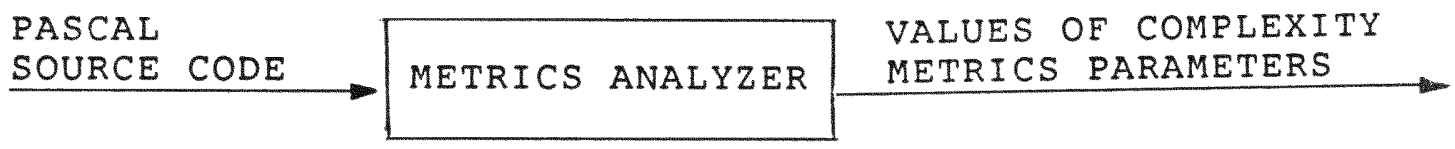

Figure 5. I/O Characteristics of the Metrics Analyzer Program

The counting and computation strategy for all the complexity metrics are straight forward. The one exception is McCabe's cyclomatic complexity, $V(G)$. which is equal to the number of predicates plus one. In order to obtain the total number of predicates, the Metrics Analyzer program was modified to count the occurrences of each type of conditional statement such as IF, WHILE, FOR, CASE and REPEAT. For each of these, it determines the number of control flow paths (e.g. an IF statement increases the total control flow path count by one). The CASE statement is handled as follows.

1. Count the number of CASE clauses (the absence of 'ELSE' or 'OTHERWISE' increases the control flow path count by 
one)

2. Substract one from it.

5.2.1.2 Methodology Used for Collecting the Information for the Cost Estimation Models

A cost estimation analysis was performed only for the FIU programs because the actual or estimated development efforts were known. In order to use the development time in the model, it was necessary to convert from school hours to man years or man months. We found that students average about ten hours a week on one project. Therefore 520 hours was used to represent a one-year working time, and this was used as a basis to convert hours to man-years or man-months for all the cost models.

The data collected could be used directly with the IBM cost model to calculate the development effort. However, for the SLIM and Cocomo models, values for the technology constant and effort multipliers were needed. In order to obtain the value for SLIM's $\mathrm{C}_{k}$ for the school environment, the constant ' $C$ ' is needed. This ' $C$ ' is used by Putnam in his simple software cost estimation system for selecting the $c_{k}$. The formula for ' $C$ ' is defined as

$C=S_{S} /(E / B)^{1 / 3}\left(T_{d}\right)^{4 / 3}$

where $\mathrm{S}_{S}$ is the number of executable source lines, $E$ and Td are the development effort in man-years and the development time in years respectively. ' $B$ ' is another constant derived by Putnam for computing ' $C$ '. Its values 
corresponding to a given project size are listed below.

\begin{tabular}{rr}
$\operatorname{SIZE}(1000$ LINES $)$ & $B$ \\
\hline $5-15$ & 0.16 \\
20 & 0.18 \\
30 & 0.28 \\
40 & 0.34 \\
50 & 0.37 \\
70 & 0.39 \\
$>=100$ & 0.39 \\
\hline
\end{tabular}

To determine $C_{k}$, the following procedure is used:

. Convert ' $\mathrm{E}$ ' and ' $\mathrm{T}$ ' to units of years. In the school environment 520 hours was used to represent one-year working time for a project.

. Determine the value of $B$. There is no published value of $B$ for projects of less than 5,000 lines required by this study and there is no published formula to calculate this quantity. Therefore the model development effort was calculated using values of 'B' ranging from 0.05 to 0.16 . We observed that 0.16 , which is the minimum value for ' $B$ ' corresponding to the source lines 5,000-10,000 produced good estimates for the development effort and hence was used in the calculation for ' $C$ ' for all projects.

. Compute ' $C$ ' from the function mentioned above.

. Select the value of $\mathrm{C}_{k}$ which is greater than and the closest to the computed ' $C$ ' from the values listed in Table 2. If no corresponding $C_{k}$ exists, than ' $C$ ' is 
used instead. The table has a range of $\mathrm{C}_{k}$ values applicable to a majority of industrial projects. Since the value of $C$ is the basis for choosing $C_{k}$, this should be a valid technique.

The determination of the ratings for the cost driver attributes was done in consultation with the professors and programmers. In the cocomo model, we basically retain Boehm's rating scale (see Tables 3 and Table 4 ) for most of the effort adjustment factors except some personnel attributes such as analysts' capability, application experience, programmer capability, virtual machine experience, and programming language experience. These constants were determined from considering the level of the course in the computer science program. For example, the introductary level PASCAL programs were rated "low" while the more sophiscated level operating system projects were rated "high" on the programming language experience attribute. Simple programs were written to do most of the needed calculations. The only exception was the calculation for the Cocomo models. An automated software package-WICOMO developed by the wang Institute of Graduate studies [27] was used. WICOMO is an interactive software cost estimation model available on IBM PC. It is based on Boehm's COCOMO model to calculate the estimates of man-month of effort, cost and schedule for a project. The major input parameters of WICOMO are project size and the ratings of environmental factors (i.e. effort multipliers). 


\subsubsection{Data Collected}

Software complexity metrics parameter values (Halstead's parameters and McCabe's cyclomatic complexity) as well as total source lines and total instructions were obtained for all programs in both groups, FIU and NCR. However, only the FIU programs were used in the analysis related to the development effort.

Tables showing input from the FIU data group:

Table 6 lists the values of complexity metrics parameters for the 54 FIU PASCAL programs. Additionally, the instruction density (ratio of the number of instructions per $V(G))$, total number of source lines, the number of instructions and the source of program are also given. Calculations for McCabe's cyclomatic complexity, $V(G)$, are shown in Table 7. The number of predicates is presented in the column labeled 'PREDICATE'. Complexity metrics values for programs obtained from the same class were averaged together. These average metrics values and the corresponding actual development effort are listed in Table 8, and marked with ' *' in the first coulmn. Table 9 lists the parameters used to obtain the predicted development effort for the SLIM model. Table 10 includes the ratings for FIU projects and the predicted development effort obtained by using the organic mode of Boehm's Intermediate Cосомо model. Table 11 gives the adjusted ratings for the personnel attributes used and gives the adjusted cocomo model predicted effort. 
Table showing input from the NCR data group:

The complexity metrics of NCR's 324 small programs are listed in Table 12. Some of the complexity metrics which were used for the effort prediction analysis (such as Halstead's potential volume, program effort and program time) are not needed for this analysis and hence are excluded from this table. 


\section{RESULTS}

Table 13 contains the summary of actual and predicted development efforts which computed by all models for 28 FIU projects. For the predicted development efforts, Table 14 gives the relative errors in estimation ( (actual - predicted) / actual) of all models for each project. It also includes the total, average and the range of the relative error of these predicted development efforts. Seven of these 28 projects are more than 1000 lines long and results for them are given in Table 15 . The regression results using linear and nonlinear models on the FIU data are presented in Table 16 and Table 17, and those for NCR data are listed in Tables 18 and 19. These values indicated that the residual mean square computed from the linear regression model are close to those from the nonlinear model. This results from the fact that the regression relationship is close to linear, the power constant P2 is close to 1 (see Table 17 and Table 19). various results regarding the previous hypotheses are discussed below:

6.1. The Effectiveness of Cost Estimation Models-IBM, SLIM, and Cocomo on Predicting Development Effort for small scale Projects

The accuracy of each model can be evaluated by comparing the actual and predicted development efforts. The analyses showed that the IBM and SLIM models usually overpredicted the actual effort whereas the cocomo model 
usually underpredicted the actual development effort (see Table 13). The average relative errors in estimation of these three models with respect to the actual development effort are $342 \%, 84 \%$, and $56 \%$ (see Table 14). The range of the relative errors across all 28 FIU projects for IBM, SLIM and Cocomo models varied from $59 \%$ to $1559 \%, 18 \%$ to $150 \%$, and $8 \%$ to $203 \%$ respectively. This indicated a large variability in the estimates in the case of small projects. The IBM model, which estimated the development effort by using the total number of source lines of code has the highest average relative error (342\%) of all the models. The SLIM model, where development effort is a function of several parameters such as the number of source lines of instruction, technology constant, and development time had an average relative error of $84 \%$; thus its accuracy is also poor. The Intermediate cocomo model contains more variables than the IBM model and had smaller average relative error of $56 \%$ than either the SLIM or the IBM models; however it was still not satisfactory.

since the accuracy of the cocomo model depends on the proper choices of the values of the effort multipliers, and since many of its parameters do not account for the environment under which the small size projects were developed particularly in school, some adjustments were attempted to adapt it to this environment. The adjusted Cocomo model was constructed by considering only six of the fifteen effort multipliers which are used in the organic mode of Boehm's Intermediate cocomo model. 
These six effort multipliers are

-applications experience,

-programmer capability,

-virtual machine experience,

-programming language experience,

. computer turnaround time,

.application type.

where, the last factor was adapted from RCA's PRICE model $[8,23]$ with a slight change to suit this project. The modified application type and its ratings for the adjusted Cocomo are as follows:

\begin{tabular}{ll} 
APPLICATION TYPE & RATINGS \\
\hline Operating Systems & Very High \\
Interactive Operations & Very High \\
String Manipulation & Nominal \\
Mathematical Operations & LOW \\
Other & Very Low
\end{tabular}

As mentioned earlier, the given ratings were based on the course level in the computer science program. Operating systems and interactive operations projects were rated 'very high' because they both involve a considerable amount of design and programming time by students. Weights were assigned for each rating. These weights were developed by Boehm for one of his effort multipliers, Product complextity (CPLX) in the Intermediate Cocomo model. We did not use his CPLX definition because this factor is determined by a set 
of sophisticated operations: program control, computation, device-dependent, and data management etc (see Table 3), which are not appropriate for a single module program in a school environment. The adjusted cocomo model had an average relative error of $52 \%$ which is not much different than the Intermediate cocomo model.

In addition to evaluating the cost estimation models, the same projects were also used to study the predicted development effort using Halstead's complexity metrics. This prediction was compared to the actual development effort, and the relative error in estimation varied between $8 \%$ to $83 \%$ with a mean relative error of $36 \%$. This result is much better than that of all the previous models. Thus the complexity metrics appear to be a useful tool for measuring the development effort for small projects.

Further analysis of this data showed that projects with program lengths over 1000 lines have a smaller range of relative errors than the others. The prior analyses were repeated using only projects with lengths between 1100 and 2700 lines. The average relative errors of all models except coсомо (which increased from 56\% to 64\%), were significantly reduced: IBM model error decreased from $342 \%$ to $195 \%$, SLIM model error decreased from $84 \%$ to $58 \%$, adjusted Cocomo model error decreased from $52 \%$ to $11 \%$, and Halstead prediction error decreased from $36 \%$ to $19 \%$ (see Table 15 ). This indicates that the adjusted Cocomo model produced the smallest average relative error, 0.11 of all. It seems that Boehm's cocomo model is more easily adaptable to a specific 
organization and it is easier to tune this model to a particular environment. As the investigation shows, none of these cost models estimates development effort with a satisfactory precision for the small size projects in the school environment. It seems that the effort equations derived from a data base composed of large size projects are not useful for predicting the development effort for small projects directly.

In addition to studying the relative prediction error for small projects, the statistical significance of the regression analyses was examined. The regression results indicate that the predicted development efforts computed by all the above models are statistically significant with a p value of less than .001 (see table 16). The plots of regression lines and the $95 \%$ confidence intervals about the regression line are shown in Figures 6 to 12 . Some statistics are summarized in Table 20 below.

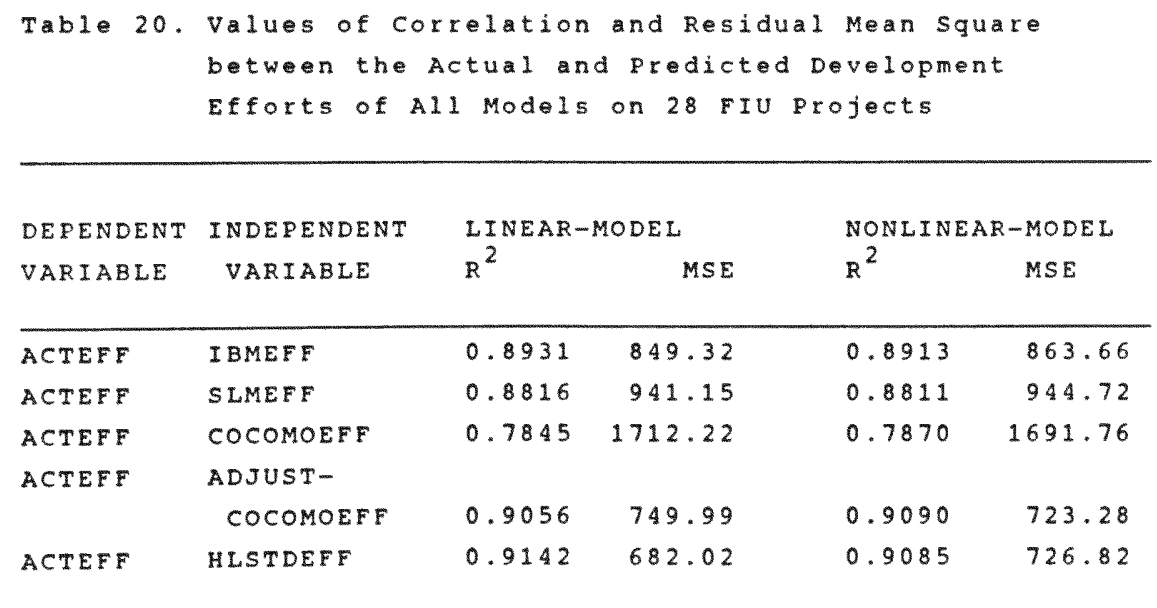


The $\mathrm{R}^{2}$ values listed in Table 20 , except for the cocomo model, are close to 90 percent using either the linear or nonlinear relationship. This means that approximately $90 \%$ of the variability in the actual development effort can be explained by the variables in the model. However the relative prediction error was quite high. In addition, the regression results also showed that the model residual mean square for the model development effort of adjusted cocomo model and Halstead are smaller than the other models. The standard errors (square root of residual mean square) of Cocomo and Halstead models were 27.38 and 26.12 hours respectively. Thus, based upon the above regression results and the average relative errors indicated in Table 14, we conclude that both Halstead and adjusted Cocomo model give better predictions of the actual development effort than the other models.

6.2. The Relationship between Actual Development Effort and McCabe's Cyclomatic Complexity.

This relationship is described by the regression results listed on Table 16 and Table 17 . The $R^{2}$ value for both the linear and non-linear models were approximately the same, 0.9003 and 0.9029 respectively. Thus the more the control paths the higher is the effort necessary for designing, coding, testing, and debugging a program, and therefore the higher is the required development effort. Thus the cyclomatic complexity is a useful measure of the required development effort. 
6.3. The Relationship between Actual Development Effort and Halstead's Potential Volume.

The $\mathrm{R}^{2}$ value for both the linear and nonlinear regression models were approximately the same, 0.7886 and 0.7871 respectively; both of which are significant. This shows that the development effort is related to the potential volume which is a measure of the difficulty of the algorithm. Although this $\mathrm{R}^{2}$ was not as high as obtained from the prior models, it does indicate that the amount of effort required is a function of the complexity of the algorithm itself. Thus in the early design phase of software development when the algorithm is designed, it is possible to obtain an estimate of the development effort.

6.4. The Relationship between the Number of Instructions (DSI), the Total Number of Distinct symbols ( $n$ ), the Program Volume (V) and the Cyclomatic complexity $(V(G))$.

These relationships can be examined from the results summarized in Table 21 below:

\begin{tabular}{|c|c|c|c|c|c|c|c|c|}
\hline \multirow[b]{2}{*}{ SOURCE } & \multicolumn{2}{|c|}{ DENDENT } & \multicolumn{2}{|c|}{ INDEPENDENT } & \multicolumn{2}{|c|}{ LINEAR-MODEL } & \multicolumn{2}{|c|}{ NONLINEAR-MODEL } \\
\hline & VARI & $A B L E$ & VARIA & $A B L E$ & $R^{2}$ & $M S E$ & $\mathrm{R}^{2}$ & MSE \\
\hline F.I.U. & $\mathrm{n}$ & & DSI & & 0.9291 & 3337.63 & 0.9175 & 3882.58 \\
\hline NCR & $n$ & & DSI & & 0.8166 & 8292.09 & 0.8604 & 6312.73 \\
\hline F.I.U. & $v$ & & DSI & & 0.9767 & 26825643.34 & 0.9877 & 14141900.00 \\
\hline NCR & $v$ & & DSI & & 0.9788 & 10332555.80 & 0.9792 & 10137100.00 \\
\hline F.I.U. & $v 1 G$ & & DSI & & 0.9084 & 730.42 & 0.9052 & 757.75 \\
\hline NCR & $v 1 G$ & & DSI & & 0.8760 & 448.72 & 0.8771 & 444.74 \\
\hline
\end{tabular}


It shows that all the $R^{2}$ values are close to 0.90 . While most of the $R^{2}$ values are close to each other there is a statistically significant difference $(p=0.002)$ between the two sources for the variables $\mathrm{n}$ and $\mathrm{DSI}$. The $\mathrm{R}^{2}$ for the FIU data of 0.9175 is higher than the NCR $R^{2}$ of 0.8604 . Thus a higher percent of the variability in $n$ was explained by DSI for the data that came from FIU. A Fisher's test of two coefficients of correlation was used for this evaluation. This could occur because all the FIU programs are composed of a single module. Every module is a complete entity which includes all the components of a project and performs a complete task. In contrast, some of the NCR programs contain only data declarations or trival function definitions which do not perform a complete task. Therefore, the relationship between DSI and $n$ varied substantially from module to module. In general, these software metrics are highly correlated with the number of instructions. The results are in agreement with our early assumptions. In addition, the results indicated that Halstead's program volume varies substantially with the number of instructions. It seems that Halstead's program volume is a very useful attribute to measure the size of a small program. Since DSI is highly correlated with these complexity metrics, and since an estimate of the number of instructions (DSI) is available in the early phase of software development, the relationships between DSI and other complexity metrics (i.e. $n, v, V(G)$ ) which are derived from historical data enable the software developers 
to obtain early information about the total effort required.

An interesting observation made from the experiment is that the mean instructions density (DSI/VG) for 54 FIU programs was 9.01 and for the 324 NCR programs was 11.53 . The instruction densities for both the NCR and FIU data were studied by dividing them into groups according to the number of lines of instruction. Table 22 below shows that the mean instruction densities of both FIU and NCR projects decreased as the size of projects increased. Thus from these limited experiments it seems that the instruction density of a program with more than 1000 source lines tends to be constant and does not depend on the project type. If the ratio $D S I / V(G)$ remains constant across different programming languages also, then this may reveal some inherent property of the languages.

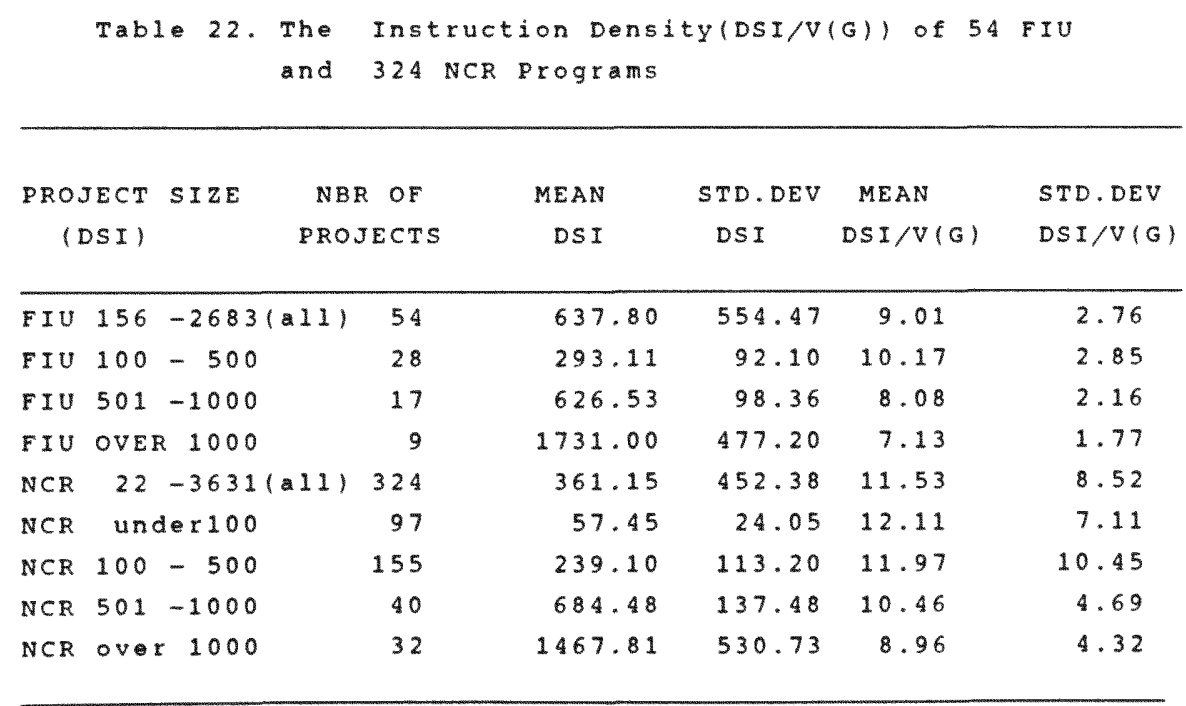




\section{CONCLUSION}

The experimental results indicate that our initial hypotheses were valid. We found that none of the formulas given by the IBM, SLIM, and COCOMO cost models accurately predicted the actual development effort for the 28 FIU projects. Apparently, environmental factors which were used by all the cost models in determing the development effort (e.g. SLIM's technology constant, cocomo's effort multipliers) are not applicable for small software product development. This is evident from the results of the adjusted cocomo model which excluded these factors and which resulted in a more accurate estimate with smaller relative error and standard error than the other cost estimation models. This was particularly true for projects larger than 1000 lines of instruction. Although the relative error for all of the models was high, the results of the regression always indicated that actual development effort is some function of the model variables. Thus the regression functions derived from using the models might be applicable for predicting software costs.

We also found for the 28 projects developed at FIU that using Halstead's effort to predict effort gave smallest standard error and the highest correlation with the actual development effort. We conclude that as compared with the large projects the development effort for small projects are more dependent on the source code itself. We also found a strong correlation between McCabe's cyclomatic complexity and the actual development effort. Hence, software 
complexity metrics seem to be reasonable measurements which can be used to estimate the amount of effort required to develop and test the software product.

This study also demonstrated that the complexity of an algorithm is related to the actual development effort. Thus, in the early software design phase, once an algorithm is specified the development effort can be predicted. Our experiments indicated that the number of source lines of instruction is strongly correlated with Halstead's vocabulary, program volume, and Mccabe's cyclomatic complexity respectively. There exist techniques to estimate lines of code at the beginning of $a$ project and hence estimate the values of various complexity metrics. Thus the regression functions derived can be used to predict values of other software attributes. The final observation from the experiments is that the instruction density of programs with lengths of more than 1000 lines of instructions appears to be constant. This shows that the instruction density can be used to estimate the DSI once the detailed design is done and $V(G)$ is available. If this estimation of DSI is accurate, then it facilitates the prediction of other software attributes related to DSI more accurately. However, this is not established conclusively by this research and is a topic for future research. 
Table 1. IBM 29 variables that correlate significantly with programming
productivity
The programing productivity is defined s the ratio of the delivered source lines
of code (DSL) to the total efort in man- months (MM). pollowing twenty-nine
variables were combined into an index based on the effect of each variable on
productivity, and the analysis was porformed on oach variable independently. It
does not take into account the possibility that these variables may be correlated.

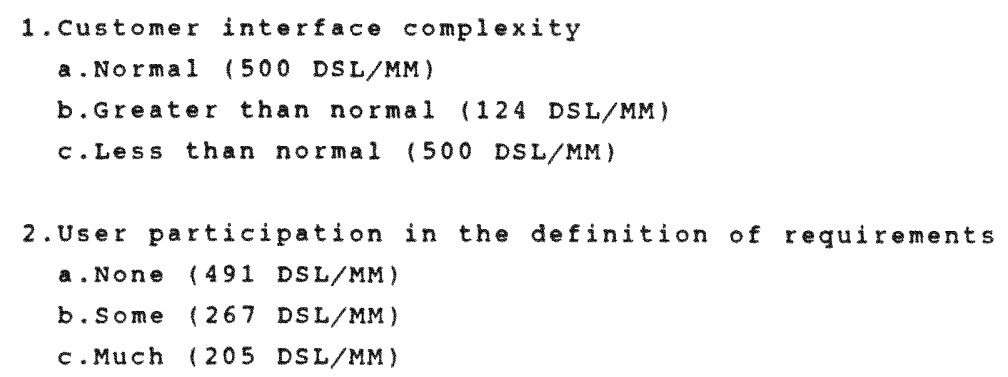




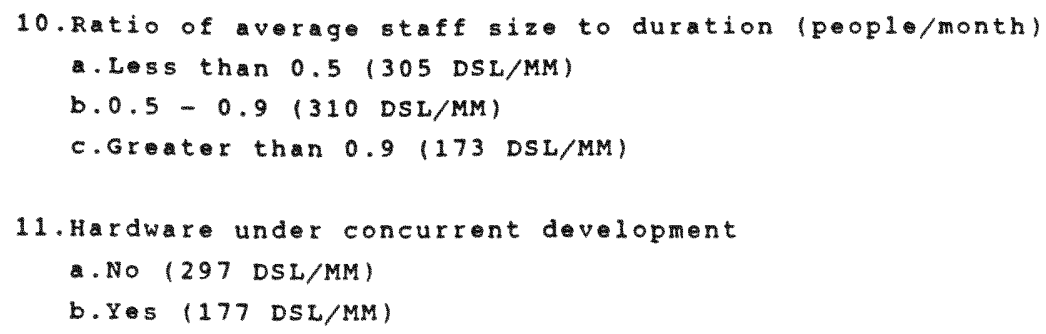




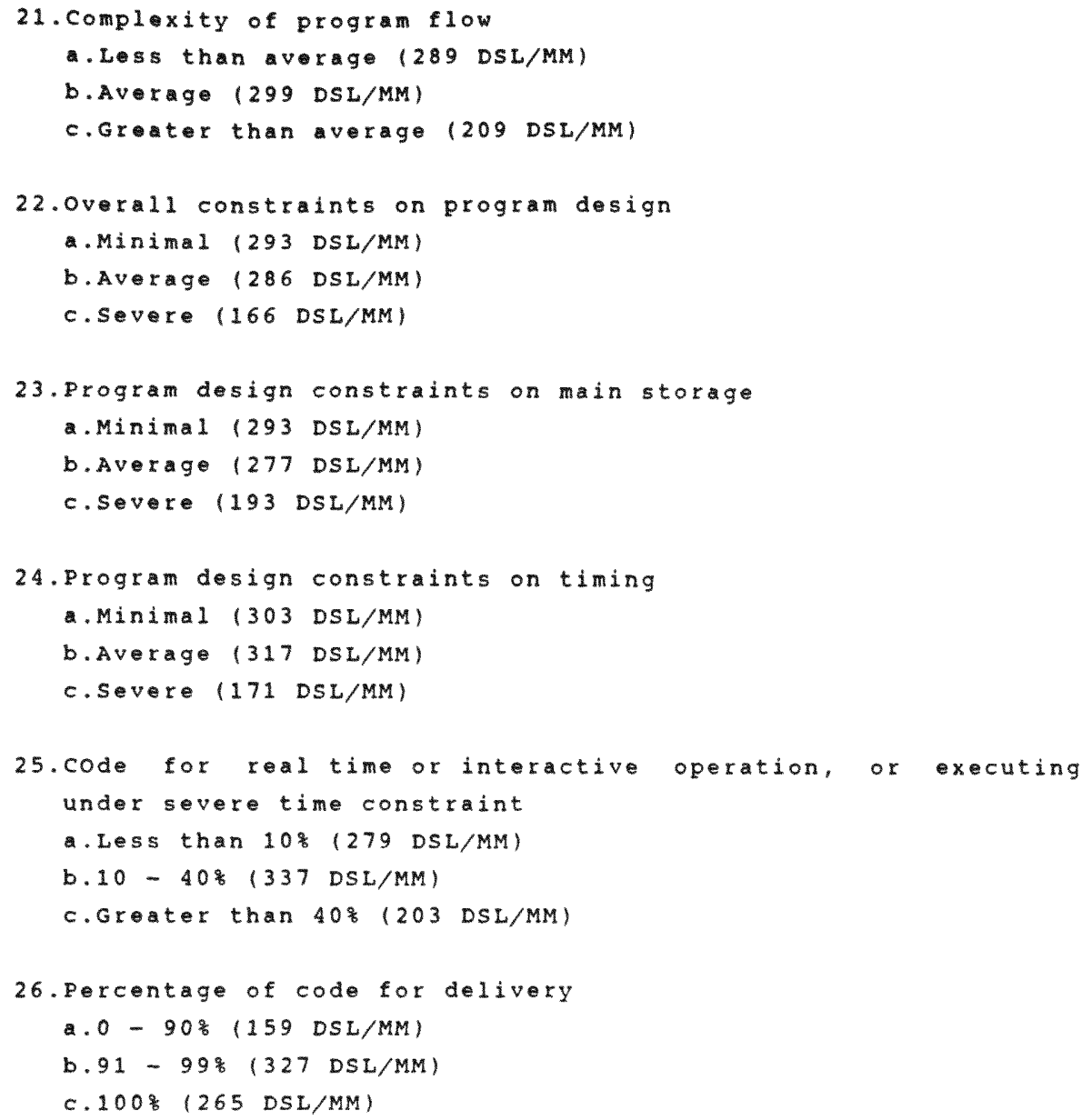


Table 2. Range of Technology constant of very simple softwar cost Estimation systom Developod by putram

\begin{tabular}{rr}
\hline NBR & RANGE OF $C_{k}$ \\
\hline 1 & 610 \\
2 & 754 \\
3 & 987 \\
4 & 1220 \\
5 & 1597 \\
6 & 1974 \\
7 & 2584 \\
8 & 3194 \\
9 & 4181 \\
10 & 5168 \\
11 & 6765 \\
12 & 8362 \\
13 & 10946 \\
14 & 13530 \\
15 & 17711 \\
16 & 21892 \\
17 & 28657 \\
18 & 35422 \\
19 & 46368 \\
\hline
\end{tabular}

where

$c_{k: \text { technology constant }}$ 
Table 3. Fiften Effort Multipliers of Boohm's Intermediate cocomo Model

Product attibuts:

RELY(Required software $r$ liability):

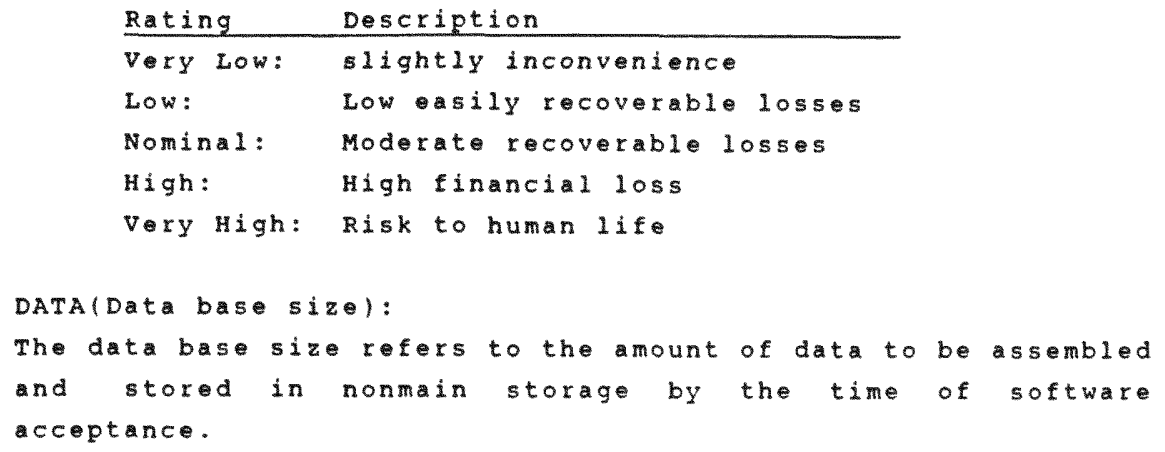




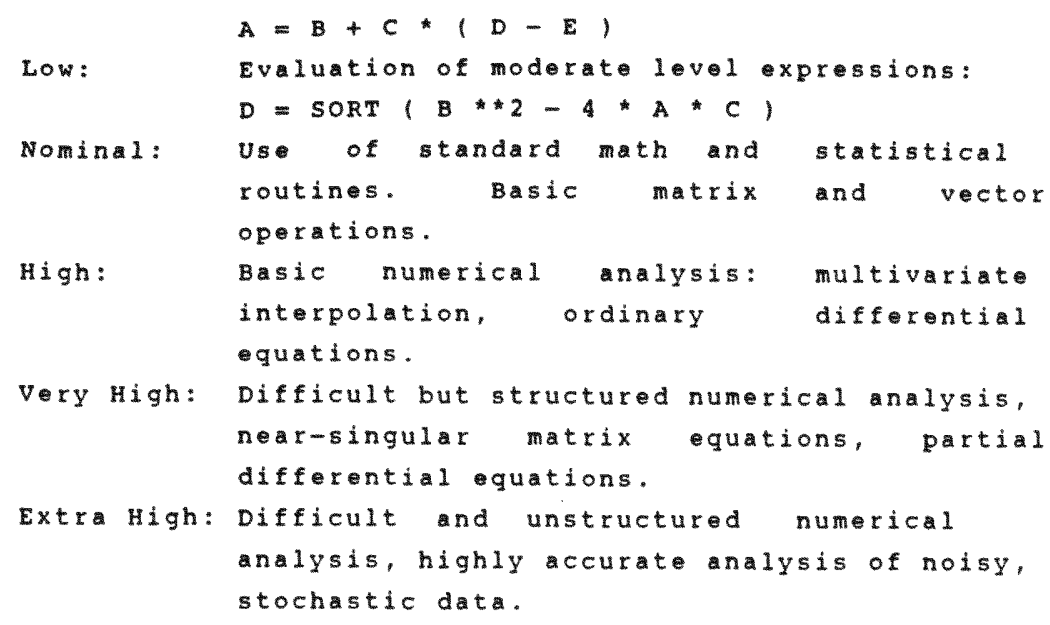

Device-dependent operations:

$\begin{array}{ll}\text { Rating } & \text { Description } \\ \text { Very Low: } & \text { Simple read, write statements with simple } \\ & \text { formats. } \\ & \text { No cognizance needed of particular processor } \\ \text { Low: } & \text { I I/O device characteristics. I/O done at } \\ & \text { GET/PUT level. No cognizance of overlap. } \\ & \text { I/O processing includes device selection, } \\ \text { Nominal: } & \text { status checking and error processing. } \\ \text { High: } & \text { Operations at physical I/O level (physical } \\ & \text { storage address translations, seeks, reads). } \\ \text { Very High: } & \text { Routine for interrupt dignosis, servicing, } \\ & \text { masking and communication line handing. } \\ \text { Extra kigh: } & \text { Device timing dependent coding, } \\ & \text { microprogrammed operations. }\end{array}$

Data management operation:

$\begin{array}{ll}\text { Rating } & \text { Description } \\ \text { Very Low: } & \text { simple arrays in main memory } \\ \text { Low: } & \text { single file subsetting with no data structure } \\ & \text { change, no edit, no intermediate file. } \\ \text { Nominal: } & \text { Multifle input and single file output. } \\ & \text { simple structural changes, simple edits. } \\ \text { High: } & \text { special purpose subroutines activated by data } \\ & \text { stream contents. Complex data restructuring } \\ & \text { at record level. generalized, parameter-driven file } \\ & \text { A gery High: } \\ & \text { structuring routine. File building, command } \\ & \text { processing, search optimization. } \\ \text { Extra High: } & \text { Highly coupled, dynamic relational structures } \\ & \text { and natural language data management. }\end{array}$

computer attributes:

TIME (Execution time constraint): 
High: $\quad 75$ th porcentile

very High: $90 \mathrm{th}$ percentile

AEXP(Applications experience):

\begin{tabular}{ll} 
Rating & Description \\
\hline Very Low: & $<=4$ months experience \\
Low: & 1 year \\
Nominal: & 3 years \\
High: & 6 years \\
Very High: & 12 years
\end{tabular}

PCAP (Programmer capability):

\begin{tabular}{ll} 
Rating & Description \\
\hline Very Low: & 15 th percentile \\
Low: & 35 th percentile \\
Nominal: & $55 t h$ percentile \\
High: & 75 th percentile \\
Very High: & 90 th percentile
\end{tabular}

VEXP(virtual machine experience):

$\begin{array}{ll}\text { Rating } & \text { Description } \\ \text { Very Low: } & <=1 \text { month experience } \\ \text { Low: } & 4 \text { months } \\ \text { Nomina 1: } & 1 \text { year } \\ \text { High: } & 3 \text { years }\end{array}$

LEXP(Programming language experience):

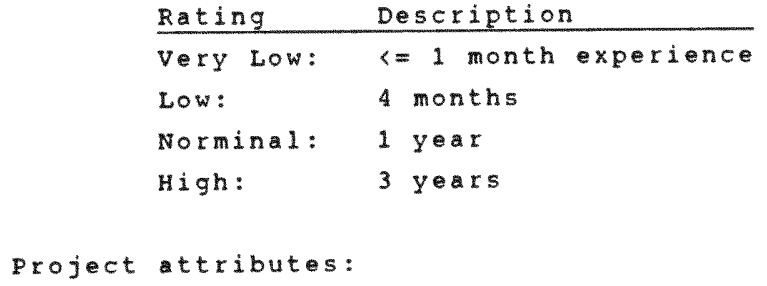


TOOL(Us of softwax tool):

Rating Description

very Low: Basic microprocessor tools

Low: Basic minicomputer tools

Nominal: Basic mini/maxi computer tools

High: Strong maxicomputer programing, test tools

very High: Advanced maxicomputer tools.

SCED(Schedule constraint):

$\begin{array}{ll}\text { Rating } & \text { Description } \\ \text { Very Low: } & 75 \% \text { (sever acceleration) } \\ \text { Low: } & 85 \% \text { (moderate accoleration) } \\ \text { Nominal: } & 1008 \text { (according to schedule) } \\ \text { High: } & 130 \% \text { (moderate stretchout) } \\ \text { Very High: } & 160 \% \text { (severe stretchout) }\end{array}$


Tablo 4. Ratings of Cocomo Dovelopment Effort Multipliers

\begin{tabular}{|c|c|c|c|c|c|c|}
\hline \multirow{3}{*}{ ATTRIBUTES } & \multicolumn{6}{|c|}{ WEIGHT } \\
\hline & \multicolumn{2}{|l|}{ VERY } & \multirow[b]{2}{*}{ NOMINAL } & \multirow[b]{2}{*}{ HIGH } & \multirow{2}{*}{$\begin{array}{l}\text { VERY } \\
\text { HIGH }\end{array}$} & \multirow{2}{*}{$\begin{array}{l}\text { EXTRA } \\
\text { HIGH }\end{array}$} \\
\hline & LoW & LoW & & & & \\
\hline RELY & .75 & .88 & 1.00 & 1.15 & 1.40 & - \\
\hline DATA & - & .94 & 1.00 & 1.08 & 1.16 & - \\
\hline CPLX & .70 & .85 & 1.00 & 1.15 & 1.30 & 1.65 \\
\hline TIME & - & - & 1.00 & 1.11 & 1.30 & 1.66 \\
\hline STOR & - & - & 1.00 & 1.06 & 1.21 & 1.56 \\
\hline VIRT & - & .87 & 1.00 & 1.15 & 1.30 & - \\
\hline TURN & - & .87 & 1.00 & 1.07 & 1.15 & - \\
\hline ACAP & 1.46 & 1.19 & 1.00 & .86 & .71 & - \\
\hline$A E X P$ & 1.29 & 1.13 & 1.00 & .91 & .82 & - \\
\hline PCAP & 1.42 & 1.17 & 1.00 & .86 & .70 & - \\
\hline VEXP & 1.21 & 1.10 & 1.00 & .90 & - & - \\
\hline LEXP & 1.14 & 1.07 & 1.00 & .95 & - & - \\
\hline MODP & 1.24 & 1.10 & 1.00 & .91 & .82 & - \\
\hline TOOL & 1.24 & 1.10 & 1.00 & .91 & .83 & - \\
\hline SCED & 1.23 & 1.08 & 1.00 & 1.04 & 1.10 & - \\
\hline
\end{tabular}

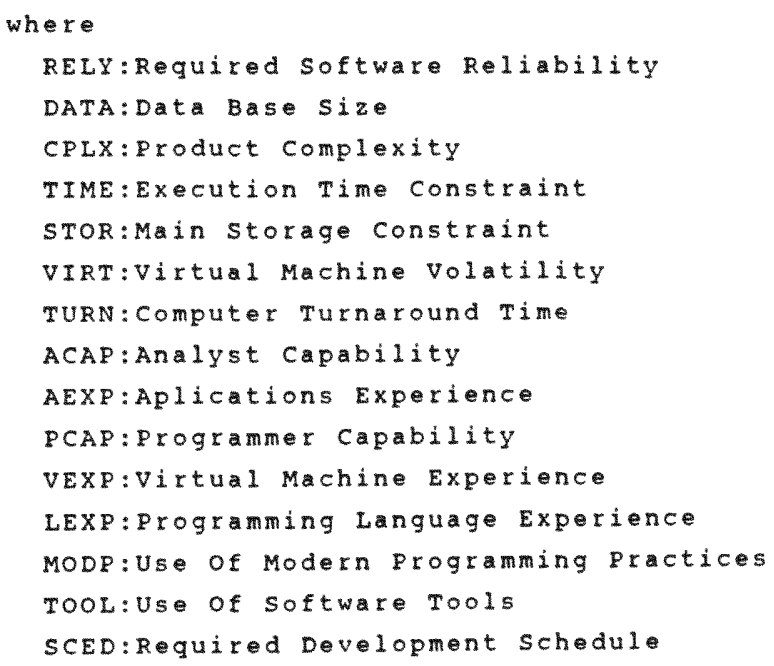


60

TABLE 5. FIU Projects' TyPes

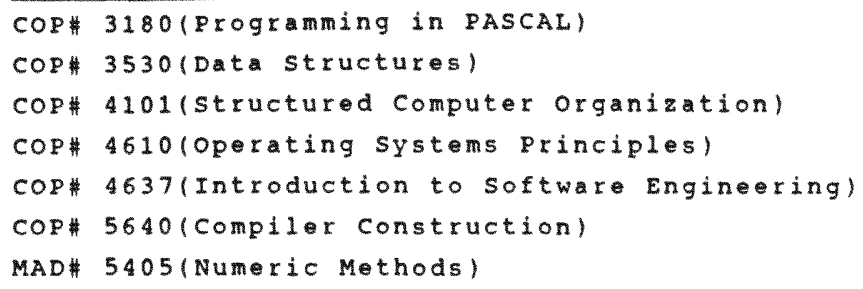




\begin{tabular}{|c|c|c|c|c|c|c|c|c|c|c|c|}
\hline NBR & DSI & SLOC & $n$ & $N$ & $v$ & $v^{*}$ & $\mathbf{E}$ & $\mathrm{T}$ & $v(G)$ & DSI/VG & CLASS \\
\hline 1 & 156 & 309 & 145 & 762 & 5471.09 & 102.66 & 291584.26 & 4.49 & 16 & 9.75 & DS. KK \\
\hline 2 & 180 & 309 & 125 & 826 & 5753.74 & 91.33 & 362485.48 & 5.59 & 21 & 8.57 & DS. LF \\
\hline 3 & 184 & 298 & 135 & 811 & 5739.30 & 98.03 & 336009.77 & 5.18 & 17 & 10.82 & DS . KK \\
\hline 4 & 194 & 261 & 108 & 936 & 6322.57 & 110.49 & 361809.33 & 5.58 & 10 & 19.40 & MACKY \\
\hline 5 & 200 & 368 & 148 & 1366 & 9631.83 & 116.34 & 797389.86 & 12.31 & 16 & 12.50 & $D S \cdot K B$ \\
\hline 6 & 200 & 304 & 129 & 1377 & 9654.46 & 88.19 & 1056855.23 & 16.31 & 20 & 10.00 & DS. KB \\
\hline 7 & 201 & 304 & 155 & 1064 & 7741.80 & 122.74 & 488318.68 & 7.54 & 17 & 11.82 & $D S . K K$ \\
\hline 8 & 201 & 519 & 144 & 1010 & 7241.62 & 140.39 & 373515.36 & 5.76 & 22 & 9.14 & DS. LB \\
\hline 9 & 208 & 257 & 156 & 1484 & 10811.54 & 129.16 & 905004.74 & 16.31 & 29 & 7.17 & $D S . K B$ \\
\hline 10 & 218 & 294 & 161 & 1452 & 10644.49 & 140.69 & 805350.65 & 12.42 & 20 & 10.90 & DS. KB \\
\hline 11 & 224 & 322 & 148 & 1016 & 7324.80 & 114.15 & 470008.30 & 7.25 & 29 & 7.72 & LOOK \\
\hline 12 & 259 & 325 & 130 & 1571 & 11302.14 & 97.19 & 1252262.79 & 19.33 & 38 & 6.82 & $\mathrm{DS}, \mathrm{KB}$ \\
\hline 13 & 269 & 356 & 172 & 1490 & 11065.13 & 163.67 & 748073.73 & 11.54 & 30 & 8.97 & DS. LG \\
\hline 14 & 291 & 362 & 179 & 1385 & 10365.08 & 148.33 & 724296.30 & 11.17 & 32 & 9.09 & DS.LG \\
\hline 15 & 309 & 420 & 178 & 1462 & 10929.52 & 174.97 & 682720.83 & 10.53 & 20 & 15.45 & OS.B \\
\hline 16 & 322 & 906 & 186 & 2291 & 17272.21 & 247.43 & 1205733.33 & 18.61 & 41 & 7.85 & SFENG \\
\hline 17 & 326 & 665 & 217 & 1801 & 13978.55 & 224.79 & 869239.35 & 13.41 & 38 & 8.58 & DS.LB \\
\hline 18 & 328 & 429 & 199 & 1898 & 14494.31 & 179.44 & 1170813.52 & 18.06 & 57 & 5.75 & DS. LB \\
\hline 19 & 328 & 429 & 202 & 1703 & 13041.93 & 192.08 & 885531.80 & 13.67 & 30 & 10.93 & DS.LG \\
\hline 20 & 342 & 838 & 291 & 1749 & 14315.34 & 288.07 & 711388.53 & 10.97 & 35 & 9.77 & ORGZN \\
\hline 21 & 358 & 371 & 140 & 1640 & 11692.02 & 118.29 & 1155707.41 & 17.83 & 46 & 7.78 & PASCL \\
\hline 22 & 387 & 451 & 162 & 1807 & 13263.11 & 159.86 & 1100389.29 & 16.98 & 38 & 10.18 & OS.B \\
\hline 23 & 392 & 679 & 268 & 2113 & 17043.65 & 243.50 & 1192981.46 & 18.41 & 33 & 11.88 & OS.LI \\
\hline 24 & 403 & 549 & 143 & 1888 & 13517.84 & 114.37 & 1597783.97 & 24.66 & 39 & 10.33 & $D S . K B$ \\
\hline 25 & 408 & 482 & 188 & 1761 & 13303.63 & 155.17 & 1140611.30 & 17.60 & 55 & 7.42 & DS. LB \\
\hline 26 & 416 & 616 & 243 & 1893 & 15001.67 & 292.86 & 768450.03 & 11.86 & 31 & 13.42 & OS.B \\
\hline 27 & 423 & 646 & 218 & 1889 & 14681.87 & 229.71 & 938376.62 & 14.48 & 32 & 13.22 & $O S . B$ \\
\hline 28 & 480 & 592 & 254 & 2476 & 19779.98 & 224.82 & 1740273.91 & 40.28 & 50 & 9.60 & UPDTE \\
\hline 29 & 502 & 922 & 241 & 2279 & 18033.48 & 216.83 & 1499798.58 & 23.14 & 54 & 9.30 & SFENG \\
\hline 30 & 512 & 655 & 244 & 2443 & 19374.79 & 236.17 & 1589478.07 & 24.52 & 53 & 9.66 & PASCL \\
\hline 31 & 513 & 854 & 185 & 2295 & 17284.52 & 153.35 & 1948162.68 & 30.06 & 86 & 5.97 & SFENC \\
\hline 32 & 535 & 668 & 330 & 2221 & 18581.60 & 330.25 & 1045499.50 & 16.13 & 71 & 7.54 & PRABU \\
\hline 33 & 550 & 977 & 276 & 2367 & 19192.88 & 278.81 & 1321202.65 & 20.39 & 41 & 13.41 & OS.M \\
\hline 34 & 554 & 945 & 203 & 2353 & 18504.43 & 253.64 & 1349986.50 & 20.83 & 51 & 10.86 & SFENG \\
\hline 35 & 593 & 917 & 242 & 2922 & 23138.92 & 222.79 & 2403240.16 & 37.09 & 93 & 6.38 & SPELL \\
\hline 36 & 620 & 697 & 268 & 3279 & 26448.71 & 204.80 & 3415611.57 & 52.71 & 116 & 5.34 & MTRXF \\
\hline 37 & 621 & 688 & 198 & 3435 & 26206.84 & 147.92 & 4643196.75 & 71.65 & 121 & 5.17 & MTRXF \\
\hline 38 & 642 & 845 & 293 & 4189 & 34327.83 & 326.71 & 3606921.75 & 55.67 & 81 & 7.93 & ORGZN \\
\hline 39 & 656 & 720 & 277 & 3000 & 24341.23 & 276.66 & 2141623.88 & 33.04 & 88 & 7.45 & PASCL \\
\hline 40 & 663 & 803 & 302 & 3481 & 28677.89 & 368.58 & 2231351.97 & 34.43 & 97 & 6.84 & PASCL \\
\hline 41 & 674 & 1292 & 242 & 4479 & 35468.59 & 253.19 & 4968600.00 & 76.67 & 68 & 9.91 & ORGZN \\
\hline 42 & 677 & 1083 & 269 & 3601 & 29065.34 & 243.48 & 3469642.88 & 53.54 & 81 & 8.36 & OS. LW \\
\hline 43 & 720 & 841 & 289 & 4238 & 34645.33 & 274.78 & 4368176.57 & 67.41 & 88 & 8.18 & PRABU \\
\hline 44 & 740 & 987 & 315 & 4162 & 34541.30 & 245.94 & 4851077.50 & 74.86 & 124 & 5.97 & MARXL \\
\hline 45 & 879 & 1566 & 557 & 5598 & 51062.34 & 668.65 & 3899431.54 & 60.18 & 97 & 9.06 & OS.HL \\
\hline 46 & 1139 & 1471 & 562 & 6260 & 57181.51 & 563.89 & 5798534.75 & 89.48 & 112 & 10.17 & PRABU \\
\hline 47 & 1309 & 1934 & 387 & 6606 & 56786.43 & 369.10 & 8736649.00 & 134.82 & 209 & 6.26 & MTRXF \\
\hline 48 & 1311 & 1579 & 436 & 6505 & 57037.04 & 415.70 & 7825830.00 & 120.77 & 231 & 5.68 & REVEW \\
\hline
\end{tabular}




$\begin{array}{rrrrrrrrrrrrrr}49 & 1653 & 2579 & 619 & 10099 & 93656.06 & 608.27 & 14420350.55 & 222.54 & 179 & 9.23 & \text { PASER } \\ 50 & 1716 & 2196 & 701 & 10564 & 99864.35 & 697.16 & 14305188.67 & 220.75 & 364 & 4.71 & \text { FSHER } \\ 51 & 1786 & 3734 & 738 & 10355 & 98657.02 & 740.63 & 13141825.43 & 202.81 & 253 & 7.06 & \text { NCRCN } \\ 52 & 1821 & 2332 & 626 & 10267 & 95380.62 & 628.70 & 14470253.18 & 223.31 & 268 & 6.79 & \text { CMP.L } \\ 53 & 2165 & 3742 & 965 & 12560 & 124524.68 & 1076.20 & 14408531.67 & 222.35 & 361 & 6.00 & \text { STEVE } \\ 54 & 2683 & 4068 & 1163 & 16655 & 169608.45 & 1283.73 & 22409023.30 & 345.82 & 325 & 8.26 & \text { CMP.C }\end{array}$

where

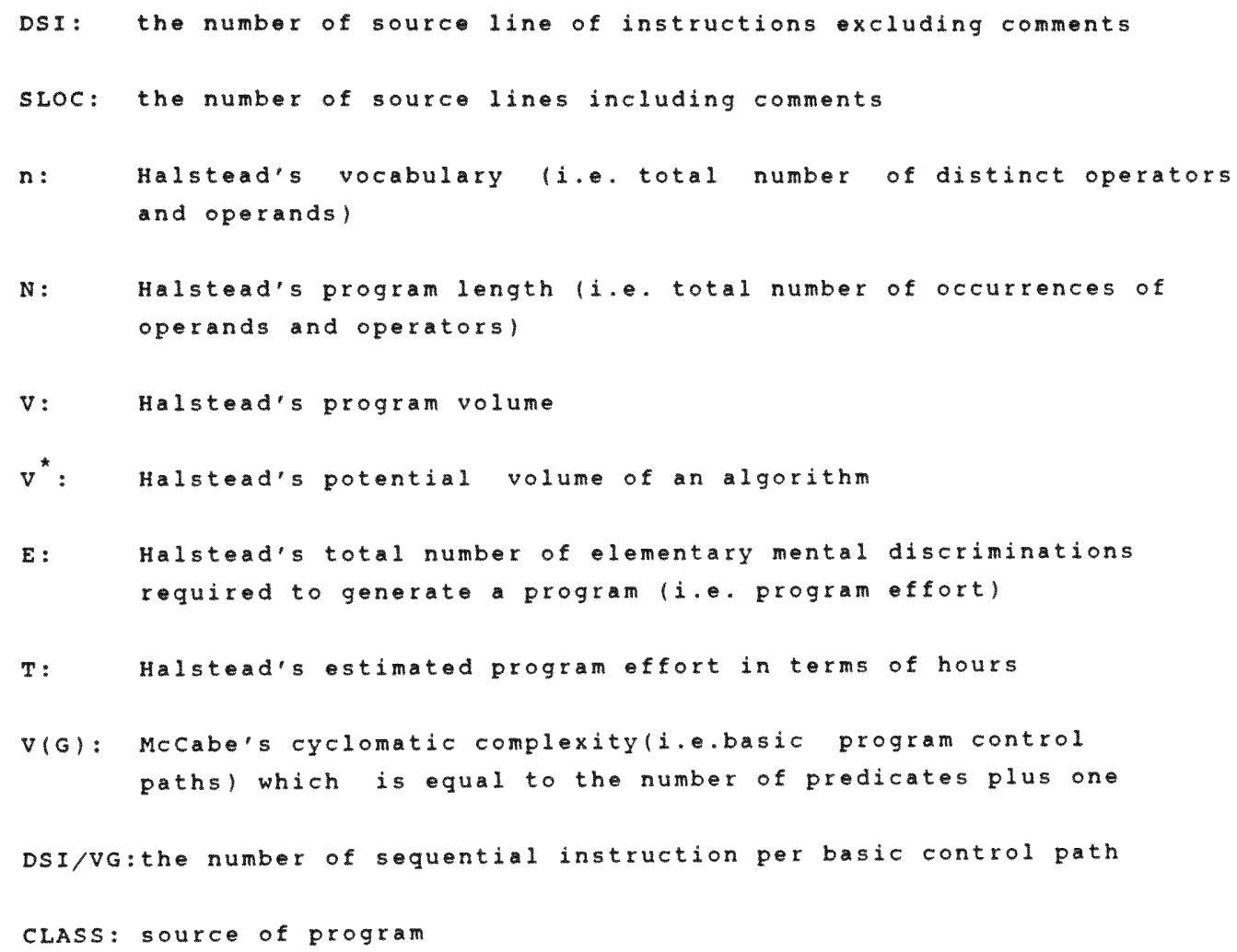


Table 7. Calculations of Mccabe's cyclomatic complexity for FIU 54 Programs

\begin{tabular}{|c|c|c|c|c|c|c|c|c|}
\hline NBR & DSI & IT & CASE & WHILE & FOR & REPEAT & PREDICATE & $v(G)$ \\
\hline 1. & 156 & 10 & - & 1 & 3 & 1 & 15 & 16 \\
\hline 2. & 180 & 14 & - & 1 & 4 & 1 & 20 & 21 \\
\hline 3. & 184 & 11 & - & 2 & 3 & - & 16 & 17 \\
\hline 4. & 194 & 4 & 3 & 2 & - & - & 9 & 10 \\
\hline 5. & 200 & 12 & - & 1 & - & 2 & 15 & 16 \\
\hline 6. & 200 & 5 & - & 2 & 10 & 2 & 19 & 20 \\
\hline 7 & 201 & 9 & - & 4 & 3 & - & 16 & 17 \\
\hline 8. & 201 & 15 & - & 6 & - & - & 21 & 22 \\
\hline 9. & 208 & 13 & - & 6 & 9 & - & 28 & 29 \\
\hline 10. & 218 & 6 & - & 3 & 10 & - & 19 & 20 \\
\hline 11. & 224 & 13 & 2 & 3 & 9 & 1 & 28 & 29 \\
\hline 12. & 259 & 22 & - & 2 & 13 & - & 37 & 38 \\
\hline 13. & 269 & 14 & - & 7 & 8 & - & 29 & 30 \\
\hline 14. & 291 & 20 & - & 1 & 6 & 4 & 31 & 32 \\
\hline 15. & 309 & 11 & - & 1 & 4 & 3 & 19 & 20 \\
\hline 16. & 322 & 30 & - & 3 & 9 & - & 40 & 41 \\
\hline 17. & 326 & 21 & - & 3 & 7 & 6 & 37 & 38 \\
\hline 18. & 328 & 46 & - & 4 & 1 & 5 & 56 & 57 \\
\hline 19. & 328 & 16 & 2 & 3 & 7 & 1 & 29 & 30 \\
\hline 20. & 342 & 8 & 12 & - & 12 & 2 & 34 & 35 \\
\hline 21. & 358 & 20 & 10 & 4 & 11 & - & 45 & 46 \\
\hline 22. & 387 & 31 & - & 3 & 3 & - & 37 & 38 \\
\hline 23. & 392 & 18 & 3 & 5 & 5 & 1 & 32 & 33 \\
\hline 24. & 403 & 16 & - & 22 & - & - & 38 & 39 \\
\hline 25. & 408 & 46 & - & 7 & 1 & - & 54 & 55 \\
\hline 26. & 416 & 23 & - & 3 & 4 & - & 30 & 31 \\
\hline 27. & 423 & 20 & - & 10 & 1 & - & 31 & 32 \\
\hline 28. & 480 & 38 & - & 7 & 4 & - & 49 & 50 \\
\hline 29. & 502 & 34 & - & 13 & 5 & 1 & 53 & 54 \\
\hline 30. & 512 & 28 & 8 & 8 & 7 & 1 & 52 & 53 \\
\hline 31. & 513 & 71 & - & 3 & 9 & 2 & 85 & 86 \\
\hline 32. & 535 & 28 & 23 & 1 & 18 & - & 70 & 71 \\
\hline 33. & 550 & 30 & - & - & 10 & - & 40 & 41 \\
\hline 34. & 554 & 35 & - & 9 & 6 & 0 & 50 & 51 \\
\hline 35. & 593 & 59 & 17 & 11 & 3 & 2 & 92 & 93 \\
\hline 36. & 620 & 55 & 15 & 8 & 29 & 8 & 115 & 116 \\
\hline 37. & 621 & 63 & - & 10 & 43 & 4 & 120 & 121 \\
\hline 38. & 642 & 57 & 5 & - & 17 & 1 & 80 & 81 \\
\hline 39. & 656 & 37 & 28 & 5 & 7 & 10 & 87 & 88 \\
\hline 40. & 663 & 30 & 29 & 3 & 33 & 1 & 96 & 97 \\
\hline 41. & 674 & 23 & 28 & 2 & 14 & - & 67 & 68 \\
\hline 42. & 676 & 56 & 8 & 9 & 7 & - & 80 & 81 \\
\hline 43. & 720 & 46 & 28 & 1 & 12 & - & 87 & 88 \\
\hline 44. & 740 & 61 & 11 & 12 & 33 & 6 & 123 & 124 \\
\hline 45. & 879 & 74 & 9 & 3 & 10 & - & 96 & 97 \\
\hline 46. & 1139 & 53 & 12 & 27 & 11 & 8 & 111 & 112 \\
\hline 47. & 1309 & 122 & - & 28 & 58 & - & 208 & 209 \\
\hline
\end{tabular}




\begin{tabular}{|c|c|c|c|c|c|c|c|c|}
\hline 48. & 1311 & 129 & 36 & 35 & 25 & 5 & 230 & 231 \\
\hline 49. & 1653 & 85 & 53 & 18 & 12 & 10 & 178 & 179 \\
\hline 50. & 1716 & 238 & 81 & 26 & 5 & 13 & 363 & 364 \\
\hline 51. & 1786 & 123 & 66 & 35 & 15 & 13 & 252 & 253 \\
\hline 52. & 1821 & 50 & 186 & 14 & 12 & 5 & 267 & 268 \\
\hline 53. & 2165 & 179 & 61 & 19 & 95 & 6 & 360 & 361 \\
\hline 54 & 2683 & 142 & 130 & 36 & 6 & 10 & 324 & 325 \\
\hline
\end{tabular}

where

DSI: the number of source line of instructions excluding comments

IF: the number of 'IF' statements in a program

CASE: the number of 'CASE'Cluses in program

WHILE: the number of 'WHILE' statements in program

FOR: the number of 'FOR' statements in a program

REPEAT: the number of 'REPEAT' statement in program

PREDICATE: total number of 'IF', 'CASE', 'WHILE', 'FOR', and

'REPEAT' in a program (i.e. total number of predicates)

$V(G)$ : Mccabe's cyclomatic complexity (i.e. program control paths) which is equal to the number of predicates plus one 
Table 8. Complexity Metries and Actual Dovelopment Effort of 28 FIU Projects

\begin{tabular}{|c|c|c|c|c|c|c|c|c|c|c|c|c|}
\hline \multicolumn{2}{|r|}{ NBR } & \multirow{2}{*}{$\frac{D S I}{180}$} & \multirow{2}{*}{$\frac{\mathrm{ACT}}{20}$} & \multirow{2}{*}{$\frac{\text { SLOC }}{304}$} & \multirow{2}{*}{$\frac{n}{145}$} & \multirow{2}{*}{$\frac{N}{879}$} & \multirow{2}{*}{$\frac{v}{6317.40}$} & \multirow{2}{*}{$\frac{v^{*}}{107.81}$} & \multirow{2}{*}{$\frac{E}{371970.90}$} & \multirow{2}{*}{$\frac{T}{5.74}$} & \multirow{2}{*}{$\frac{v(G)}{16}$} & \multirow{2}{*}{$\frac{\text { CLASS }}{\text { DS.KK }}$} \\
\hline * & 1. & & & & & & & & & & & \\
\hline & 2. & 180 & 9 & 309 & 125 & 826 & 5753.74 & 91.33 & 362485.48 & 5.59 & 21 & DS.LF \\
\hline & 3 & 194 & 4 & 261 & 108 & 936 & 6322.57 & 110.49 & 361809.33 & 5.58 & 10 & MACKY \\
\hline & 4. & 224 & 30 & 322 & 148 & 1016 & 7324.80 & 114.15 & 470008.30 & 7.25 & 29 & LOOK \\
\hline \multirow[t]{2}{*}{ * } & 5 & 248 & 15 & 350 & 145 & 1518 & 9125.13 & 92.80 & 1069107.89 & 16.66 & 29 & DS.KB \\
\hline & 6. & 296 & 18 & 382 & 184 & 1526 & 11490.71 & 168.02 & 785967.27 & 12.13 & 31 & DS. LG \\
\hline * & 7. & 316 & 22 & 524 & 187 & 1618 & 12254.53 & 174.95 & 888544.88 & 13.71 & 43 & $D S \cdot L B$ \\
\hline & 8 & 384 & 80 & 533 & 201 & 1763 & 13469.04 & 214.35 & 872484.19 & 13.46 & 30 & OS.BK \\
\hline & 9. & 392 & 40 & 679 & 268 & 2113 & 17043.65 & 243.50 & 1192981.46 & 18.41 & 33 & OS.LI \\
\hline \multirow[t]{3}{*}{ * } & 10 & 473 & 80 & 907 & 204 & 2304 & 17773.66 & 217.79 & 1500920.27 & 23.16 & 58 & SFENG \\
\hline & 11. & 480 & 50 & 592 & 254 & 2476 & 19779.98 & 224.82 & 1740273.91 & 40.28 & 50 & UPDTE \\
\hline & 12. & 535 & 30 & 668 & 330 & 2221 & 18581.60 & 330.25 & 1045499.50 & 16.13 & 71 & PRABU \\
\hline \multirow[t]{3}{*}{ * } & 13 & 547 & 20 & 637 & 240 & 2641 & 21021.48 & 249.93 & 1779540.33 & 27.46 & 71 & PASCL \\
\hline & 14. & 550 & 40 & 977 & 276 & 2367 & 19192.88 & 278.81 & 1321202.65 & 20.39 & 41 & OS.M \\
\hline & 15. & 593 & 90 & 917 & 242 & 2922 & 23138.92 & 222.79 & 2403240.16 & 37.09 & 93 & SFELL \\
\hline & 16. & 658 & 40 & 1069 & 268 & 4334 & 34898.21 & 289.95 & 4287760.88 & 66.17 & 75 & ORGZN \\
\hline & 17. & 677 & 90 & 1083 & 269 & 3601 & 29065.34 & 243.48 & 3469642.88 & 53.54 & 81 & OS.LW \\
\hline & 18. & 720 & 60 & 841 & 289 & 4238 & 34645.33 & 274.78 & 4368176.57 & 67.41 & 88 & PRABU \\
\hline & 19. & 740 & 60 & 987 & 315 & 4162 & 34541.30 & 245.94 & 4851077.50 & 74.86 & 124 & MARXL \\
\hline \multirow[t]{9}{*}{ * } & 20. & 823 & 80 & 1077 & 292 & 4371 & 35995.82 & 241.94 & 5411633.70 & 83.51 & 142 & MTRXE \\
\hline & 21 & 879 & 70 & 1566 & 557 & 5598 & 51062.34 & 668.65 & 3899431.54 & 60.18 & 97 & OS.HL \\
\hline & 22.1 & 139 & 60 & 1471 & 562 & 6260 & 57181.51 & 563.89 & 5798534.75 & 89.48 & 112 & PRABU \\
\hline & 23.1 & 311 & 160 & 1579 & 436 & 6505 & 57037.04 & 415.70 & 7825830.00 & 120.77 & 231 & REVEW \\
\hline & 24.1 & 1653 & 200 & 2579 & 619 & 10099 & 93656.06 & 608.27 & 14420350.55 & 222.54 & 179 & PASER \\
\hline & 25.17 & 1786 & 225 & 3734 & 738 & 10355 & 98657.02 & 740.63 & 13141825.43 & 202.81 & 253 & $N C R C N$ \\
\hline & 26.18 & 821 & 250 & 2332 & 626 & 10267 & 95380.62 & 628.70 & 14470253.18 & 223.31 & 268 & CMP.I \\
\hline & 27.21 & 165 & 275 & 3742 & 965 & 12560 & 124524.68 & 1076.20 & 14408531.67 & 222.35 & 361 & STEVE \\
\hline & 28.26 & 2683 & 320 & 4068 & 1163 & 16655 & 169608.45 & 1283.73 & 22409023.30 & 345.82 & 325 & $C M P \cdot C$ \\
\hline
\end{tabular}

where

DSI: the number of source line of instructions excluding comments

sLoC: the number of total source lines including comments

ACT: actual development effort

n: Halstead's vocabulary size li.e. total number of distinct operators and operands)

N: Halstead's program langth (i.e. total number of occurrences of operators and operands)

V: Halstead's program volume

$V^{*}$ : Halstead's potential volume

E: Halstead's total number of elementary mental discriminations required to generate program (i.e. program effort)

T: Halstead's program fofortin torms of hours

$v(G)$ : Mccabe's cyclomatic complexity (i.e. program control paths) which is equal to the number of predicates plus one

CLASS: source of program 


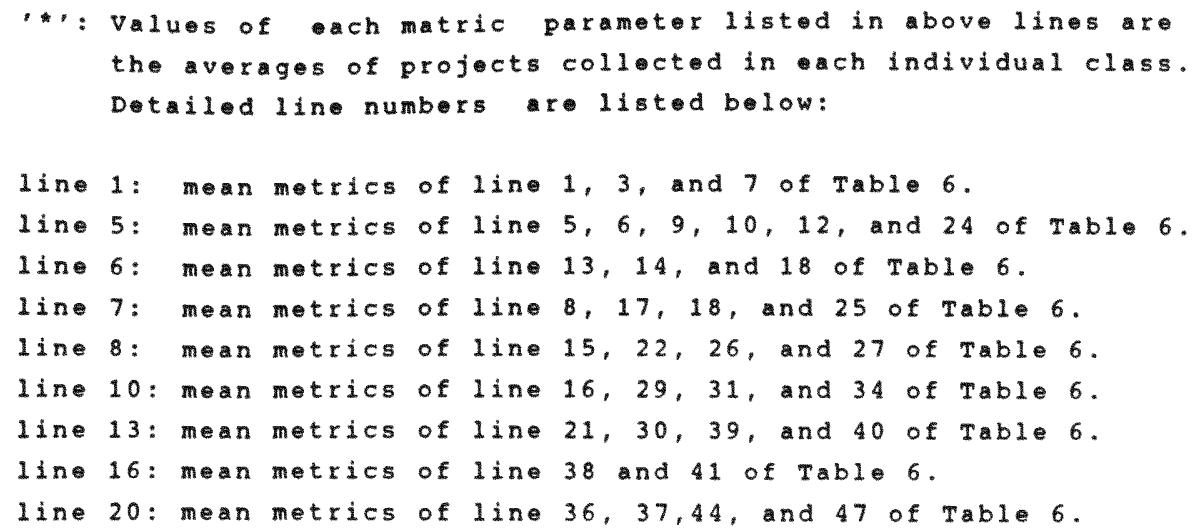


Table 9. Calculated Tochnology Constant and Technology Constant Derived by putnam for 28 FIU projects

\begin{tabular}{|c|c|c|c|c|c|c|}
\hline NBR & DSI & $\mathrm{T}_{\mathrm{d}}$ & $c$ & & $c_{k}$ & ESTM \\
\hline 1 & 180 & 9 & 84381 & 棤 & 84381 & 22.50 \\
\hline 2 & 180 & 20 & 22298 & & 28657 & 23.55 \\
\hline 3 & 194 & 4 & 351355 & \# & 351355 & 20.00 \\
\hline 4 & 224 & 30 & 14117 & & 17711 & 37.98 \\
\hline 5 & 248 & 15 & 49622 & \# & 49622 & 37.50 \\
\hline 6 & 296 & 18 & 43706 & & 46368 & 37.69 \\
\hline 7 & 316 & 22 & 33396 & & 35422 & 46.09 \\
\hline 8 & 384 & 80 & 4719 & & 5168 & 152.32 \\
\hline 9 & 392 & 40 & 15295 & & 17711 & 64.41 \\
\hline 10 & 473 & 80 & 5813 & & 6765 & 126.91 \\
\hline 11 & 480 & 50 & 12912 & & 13530 & 108.64 \\
\hline 12 & 535 & 30 & 33718 & & 35422 & 64.69 \\
\hline 13 & 547 & 20 & 67761 & 粠 & 67761 & 50.00 \\
\hline 14 & 550 & 40 & 21460 & & 21892 & 94.20 \\
\hline 15 & 593 & 90 & 5989 & & 6765 & 156.12 \\
\hline 16 & 658 & 40 & 25674 & & 28657 & 71.92 \\
\hline 17 & 677 & 90 & 6837 & & 8362 & 123.01 \\
\hline 18 & 720 & 60 & 14293 & & 17711 & 78.83 \\
\hline 19 & 740 & 60 & 14690 & & 17711 & 85.59 \\
\hline 20 & 823 & 80 & 10144 & & 10946 & 157.82 \\
\hline 21 & 879 & 70 & 13495 & & 13530 & 173.68 \\
\hline 22 & 1139 & 60 & 22610 & & 28657 & 73.68 \\
\hline 23 & 1311 & 160 & 5075 & & 5168 & 378.82 \\
\hline 24 & 1653 & 200 & 4411 & & 5168 & 311.03 \\
\hline 25 & 1786 & 225 & 3917 & & 4181 & 462.54 \\
\hline 26 & 1821 & 250 & 3350 & & 4181 & 321.66 \\
\hline 27 & 2165 & 275 & 3398 & & 4181 & 369.21 \\
\hline 28 & 2638 & 320 & 3271 & & 4181 & 383.26 \\
\hline
\end{tabular}

\footnotetext{
where

DSI: the number of source line of instructions excluding comments

$T_{d}$ : the development time in hours

c: technology constant comupted by using the equation developed by putnam for simple software cost estimation system. It defines as$$
c=s /(E / B)^{1 / 3}\left(\mathrm{~T}_{\mathrm{d}}\right)^{4 / 3}
$$

$c_{k}$ : technology constant derived by putnam

ESTM: estimated development effort (hours) computed from putnam SLIM's mode 1

* represents the value of $c_{k}$ beyonds the range of original technology constant derived by putnam (see table 2)
} 
Tabl 10. Ratings of the organic Mode of Boehm's Intermediate cocomo Model for 28 Fu projects

NBR DSI CLASS RL DT CP TM ST VR TR AC AE PC VE LE MD TL SC ESTM

\begin{tabular}{|c|c|c|c|c|c|c|c|c|c|c|c|c|c|c|c|c|c|c|}
\hline 1 & 180 & DS. KK & $V L$ & $\mathbf{N}$ & $V L$ & $\mathbf{N}$ & $\mathbb{N}$ & $L$ & L & $\mathbb{N}$ & VL & $\mathbb{N}$ & $\mathrm{N}$ & $\mathrm{N}$ & $\mathbf{N}$ & $N$ & $\mathrm{~N}$ & 13.00 \\
\hline 2 & 180 & $D S . L F$ & $V L$ & $\mathbf{N}$ & VL & $\mathrm{N}$ & $\mathrm{N}$ & L & L & $\mathrm{N}$ & VL & $\mathbf{N}$ & $\mathrm{N}$ & $\mathrm{N}$ & N & $\mathbb{N}$ & N & 13.00 \\
\hline 3 & 194 & MACKY & VL & $\mathrm{N}$ & VL & $\mathrm{N}$ & N & $\mathbf{L}$ & L & VH & $\mathbb{N}$ & VH & H & $\mathrm{H}$ & $\mathbf{N}$ & N & $\mathbf{N}$ & 4.33 \\
\hline 4 & 224 & LOOK & VL & $\mathbf{N}$ & VL & $\mathrm{N}$ & $\mathrm{N}$ & $L$ & L & $\mathrm{H}$ & $L$ & $\mathrm{H}$ & H & H & $\mathrm{N}$ & $\mathrm{N}$ & $\mathfrak{N}$ & 8.67 \\
\hline 5 & 248 & DS.KB & VL & N & $V L$ & $\mathbb{N}$ & $\mathbf{N}$ & $L$ & $L$ & $\mathrm{~N}$ & VL & $\mathbf{N}$ & $\mathrm{N}$ & $\mathrm{N}$ & $\mathrm{N}$ & $\mathbb{N}$ & $\mathbf{N}$ & 17.33 \\
\hline 6 & 296 & DS.LG & VL & $\mathrm{N}$ & VL & $\mathfrak{N}$ & $\mathrm{N}$ & L & $\mathbf{L}$ & $N$ & VL & $N$ & $\mathrm{~N}$ & $\mathrm{~N}$ & $\mathbb{N}$ & $\mathrm{N}$ & $\mathbb{N}$ & 21.67 \\
\hline 7 & 316 & DS. LB & VL & $N$ & VL & $N$ & $\mathbf{N}$ & L & L & $\mathbb{N}$ & VL & $\mathbf{N}$ & $\mathbf{N}$ & $\mathbb{N}$ & $\mathrm{N}$ & $\mathrm{N}$ & $N$ & 21.67 \\
\hline 8 & 384 & OS. BK & VL & $\mathbb{N}$ & VL & $\mathrm{N}$ & N & $L$ & $L$ & $\mathrm{H}$ & VI & H & $\mathbf{N}$ & $\mathrm{H}$ & $\mathbb{N}$ & $\mathrm{N}$ & $N$ & 17.33 \\
\hline 9 & 392 & OS.LI & VL & $\mathbb{N}$ & VL & $\mathrm{N}$ & $\mathrm{N}$ & $L$ & L & H & VL & $\mathrm{H}$ & $\mathrm{H}$ & $\mathrm{H}$ & $\mathbb{N}$ & $\mathbf{N}$ & $\mathbf{N}$ & 17.33 \\
\hline 10 & 473 & SFENG & VL & $\mathbb{N}$ & VL & $\mathbb{N}$ & $\mathrm{N}$ & $L$ & $\mathbf{L}$ & H & VL & H & $H$ & H & $N$ & $\mathrm{~N}$ & $N$ & 21.67 \\
\hline 11 & 480 & UPDTE & VL & $\mathbf{N}$ & VI & $N$ & $\mathrm{~N}$ & L & $\mathrm{L}$ & $H$ & $\mathrm{~L}$ & $\mathrm{KH}$ & $H$ & $H$ & $\mathrm{~N}$ & $N$ & $N$ & 17.33 \\
\hline 12 & 535 & PRABU & VL & $\mathrm{N}$ & VL & $\mathbf{N}$ & $N$ & L & $\mathbf{L}$ & VH & $L$ & VH & H & $H$ & $N$ & $\mathrm{~N}$ & $N$ & 13.00 \\
\hline 13 & 547 & PASCL & VL & $\mathbf{N}$ & VL & $\mathrm{N}$ & $N$ & $\mathcal{L}$ & $\mathrm{L}$ & L. & VL & $L$ & L & $\mathbf{L}$ & $\mathbb{N}$ & $\mathbb{N}$ & $\mathrm{N}$ & 60.67 \\
\hline 14 & 550 & OS.MK & VL & $\mathrm{N}$ & VL & N & $\mathbb{N}$ & L & $\mathrm{L}$ & $\mathrm{H}$ & VL & $\mathrm{H}$ & $\mathbb{N}$ & H & $N$ & $\mathrm{~N}$ & $\mathrm{~N}$ & 26.00 \\
\hline 15 & 593 & SPELL & VL & $N$ & VL & $\mathrm{N}$ & $\mathbf{N}$ & L & L & $\mathbb{N}$ & VL & $N$ & $\mathrm{~N}$ & $N$ & $N$ & $N$ & $N$ & 43.33 \\
\hline 16 & 658 & ORGZN & VL & $\mathbf{N}$ & VL & $N$ & $\mathrm{~N}$ & $\mathcal{L}$ & $L$ & $\mathbb{N}$ & VL & $N$ & $\mathrm{~N}$ & $\mathbb{N}$ & $\mathrm{N}$ & $\mathrm{N}$ & $\mathrm{N}$ & 47.67 \\
\hline 17 & 677 & OS.LW & VL & $\mathrm{N}$ & VL & $\mathbf{N}$ & $\mathrm{N}$ & L & $L$ & $\mathbf{N}$ & VL & $\mathbb{N}$ & $H$ & $\mathrm{H}$ & $\mathrm{N}$ & $\mathbb{N}$ & $\mathrm{N}$ & 39.00 \\
\hline 18 & 720 & PRABU & VL & $N$ & VL & $N$ & $\mathrm{~N}$ & L & L & $\mathrm{VH}$ & $\mathcal{L}$ & $\mathrm{VH}$ & $\mathrm{H}$ & $\mathrm{H}$ & $\mathbb{N}$ & $\mathrm{N}$ & $\mathrm{N}$ & 17.33 \\
\hline 19 & 740 & MARXL & VL & $N$ & VL & $N$ & $\mathrm{~N}$ & $L$ & $L$ & VH & VL & VH & H & $\mathrm{H}$ & $\mathrm{N}$ & $N$ & $N$ & 21.67 \\
\hline 20 & 823 & MTRXF & VL & $\mathbf{N}$ & VL & $N$ & $\mathbf{N}$ & L & $L$ & $\mathrm{H}$ & VL & $\mathrm{H}$ & H & $\mathrm{H}$ & $\mathbf{N}$ & $\mathbf{N}$ & $\mathbf{N}$ & 39.00 \\
\hline 21 & 879 & OS.HL & VL & $\mathrm{N}$ & VL & $\mathrm{N}$ & $\mathbb{N}$ & $L$ & $L$ & $\mathrm{H}$ & VL & $\mathbf{H}$ & H & $\mathrm{H}$ & $N$ & $N$ & $N$ & 39.00 \\
\hline 22 & 1139 & PRABU & VL & $\mathrm{N}$ & VL & N & $\mathbf{N}$ & $\mathcal{L}$ & $L$ & VH & L & VH & $\boldsymbol{H}$ & H & $\mathbf{N}$ & $N$ & $N$ & 30.33 \\
\hline 23 & 1311 & REVEW & VL & $N$ & VL & $\mathbb{N}$ & $\mathbf{N}$ & $L$ & $\mathcal{L}$ & $\mathrm{H}$ & L & H & $\mathrm{H}$ & H & $N$ & $\mathrm{~N}$ & $\mathrm{~N}$ & 52.00 \\
\hline 24 & 1653 & PASER & VL & $\mathrm{N}$ & VL & $N$ & $\mathbb{N}$ & L & $\mathbf{L}$ & $\mathrm{H}$ & VL & $\mathrm{H}$ & $\mathrm{H}$ & $\mathrm{H}$ & $\mathrm{N}$ & $\mathbb{N}$ & $N$ & 78.00 \\
\hline 25 & 1786 & NCRCN & VL & $\mathbf{N}$ & VL & $\mathrm{N}$ & $\mathbf{N}$ & L & I & VH & L & VH & $H$ & H & $\mathrm{N}$ & $N$ & $N$ & 47.67 \\
\hline 26 & 1821 & $C M P \cdot L$ & VL & $N$ & VL & N & $\mathrm{N}$ & $L$ & $L$ & $\mathrm{H}$ & VL & H & H & H & $N$ & $N$ & $\mathrm{~N}$ & 86.67 \\
\hline 27 & 2165 & STEVE & VL & $\mathbf{N}$ & VL & $\mathrm{N}$ & $N$ & $\mathcal{L}$ & $L$ & $H$ & VL & H & H & н & $\mathbb{N}$ & $\mathbb{N}$ & $\mathbf{N}$ & 104.00 \\
\hline 28 & 2683 & $C M P \cdot C$ & VL & $\mathrm{N}$ & VL & $\mathbf{N}$ & $\mathbb{N}$ & L & L & $\mathrm{H}$ & VL & $H$ & $\mathrm{H}$ & н & $\mathbf{N}$ & $N$ & $\mathbb{N}$ & 130.00 \\
\hline
\end{tabular}

where

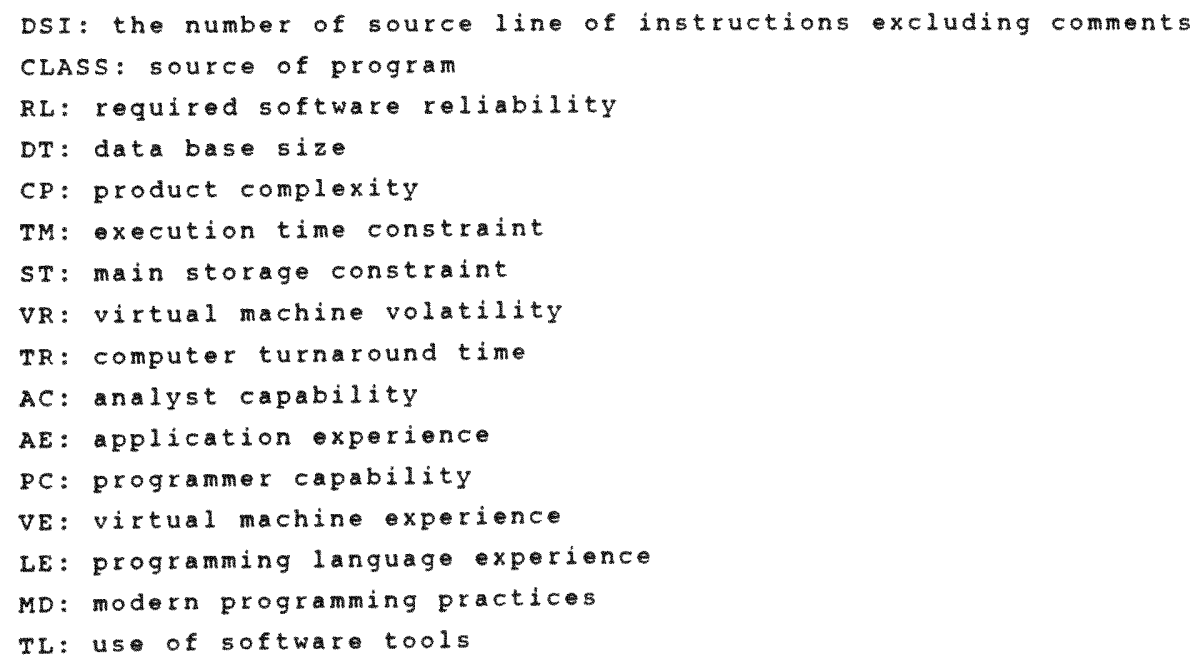


SC: $r$ quired development schedule

ESTM: -stimated development eforthours) computed from the organic mode of Boehm's Intermediat cocomo model.

VL: very low

L: $10 \mathrm{w}$

N: nomina 1

H: high

VH: very high 
Table 11. Ratings of six Effort Multipliers of Adjusted Cocomo Model for 28 FIU Project:

\begin{tabular}{|c|c|c|c|c|c|c|c|c|c|c|c|}
\hline DSI & $\mathrm{ACT}$ & CLASS & $\mathrm{AEXP}$ & PCAP & VEXP & LEXP & TURN & MM & HRS & $T Y P$ & EsTM \\
\hline 180 & 20 & DS.KK & VL & $\mathrm{N}$ & $\mathrm{N}$ & $N$ & L & 0.6 & 26.00 & VL & 18.20 \\
\hline 180 & 9 & DS.LF & VL & $\mathbb{N}$ & $\mathrm{N}$ & $\mathrm{N}$ & L & 0.6 & 26.00 & VL & 18.20 \\
\hline 194 & 4 & MACKX & $\mathrm{N}$ & $\mathrm{VH}$ & H & H & $L$ & 0.3 & 13.00 & $\mathrm{~N}$ & 13.00 \\
\hline 224 & 30 & LOOK & $L$ & $\mathrm{H}$ & H & $\mathrm{H}$ & L & 0.5 & 21.67 & VH & 28.17 \\
\hline 248 & 15 & DS.KB & VL & $\mathbb{N}$ & $\mathrm{N}$ & $\mathrm{N}$ & L & 0.8 & 34.67 & VL & 24.27 \\
\hline 296 & 18 & DS. IG & VL & $\mathfrak{N}$ & $\mathrm{N}$ & $\mathrm{N}$ & $L$ & 1.0 & 43.33 & VL & 30.33 \\
\hline 316 & 22 & DS. LB & VL & $\mathbb{N}$ & $\mathrm{N}$ & $\mathrm{N}$ & L & 1.0 & $43 \cdot 33$ & VH & 56.33 \\
\hline 384 & 80 & OS.BK & VL & $\mathrm{H}$ & $\mathbf{N}$ & $\mathrm{H}$ & $\mathbf{L}$ & 1.1 & 47.67 & $\mathrm{VH}$ & 61.97 \\
\hline 392 & 40 & OS.LI & VL & H & $\mathrm{H}$ & $\mathrm{H}$ & $L$ & 1.0 & 43.33 & VH & 56.33 \\
\hline 473 & 80 & SFENG & VL & H & H & H & L & 1.2 & 52.00 & VH & 67.60 \\
\hline 480 & 50 & UPDTE & L & H & $H$ & $\mathrm{H}$ & L & 1.0 & 43.33 & VL & 30.33 \\
\hline 535 & 30 & PRABU & $L$ & $\mathrm{VH}$ & $\mathrm{H}$ & H & L & 1.0 & 43.33 & VL & 30.33 \\
\hline 547 & 20 & PASCL & $V L$ & $L$ & L & L & L & 2.5 & 108.33 & $V L$ & 75.83 \\
\hline 550 & 40 & OS.MK & VL & $\mathrm{H}$ & $\mathrm{N}$ & H & L & 1.5 & 65.00 & VH & 84.50 \\
\hline 593 & 90 & SPELL & VL & $N$ & $\mathbf{N}$ & $\mathrm{N}$ & L & 2.0 & 86.67 & VH & 112.67 \\
\hline 658 & 40 & ORGZN & VL & $\mathrm{N}$ & $\mathrm{N}$ & $\mathbb{N}$ & $L$ & 2.2 & 95.33 & VI & 66.73 \\
\hline 677 & 90 & OS.LW & VL & $\mathrm{N}$ & H & H & $L$ & 2.0 & 86.67 & VH & 112.67 \\
\hline 720 & 60 & PRABU & $L$ & VH & $H$ & H & $L$ & 1.3 & 56.33 & VL & 39.43 \\
\hline 740 & 60 & MARXL & VL & vH & $H$ & $\mathrm{H}$ & L & 1.6 & 69.33 & $L$ & 58.93 \\
\hline 823 & 80 & MTRXF & VL & H & $H$ & $H$ & $L$ & 2.1 & 91.00 & L & 77.35 \\
\hline 879 & 70 & OS.HL & VL & H & $\mathrm{H}$ & $\mathrm{H}$ & $\mathrm{L}$ & 2.3 & 99.67 & VH & 129.57 \\
\hline 1139 & 60 & PRABU & $L$ & VH & $\mathrm{H}$ & $\mathrm{H}$ & $L$ & 2.2 & 95.33 & VL & 66.73 \\
\hline 1311 & 160 & REVEW & I & $\mathrm{H}$ & $H$ & $\mathrm{H}$ & $L$ & 3.0 & 130.00 & VH & 169.00 \\
\hline 1653 & 200 & PASER & VL & H & H & $H$ & L & 4.4 & 190.67 & $\mathrm{~N}$ & 190.67 \\
\hline 1786 & 225 & $\mathrm{NCRCN}$ & $L$ & VH & $\mathrm{H}$ & H & L & 3.5 & 151.67 & $\mathbb{N}$ & 151.67 \\
\hline 1821 & 250 & $C M P, L$ & VI & $H$ & $\mathrm{H}$ & $\mathrm{H}$ & L & 4.9 & 212.33 & $\mathrm{~N}$ & 212.33 \\
\hline 2165 & 275 & STEVE & $\mathrm{VL}$ & $\mathrm{H}$ & $H$ & $\mathrm{H}$ & L & 5.8 & 251.33 & $\mathrm{~N}$ & 251.33 \\
\hline 2683 & 320 & $C M P \cdot C$ & VL & $\mathrm{H}$ & H & $\mathrm{H}$ & $\mathcal{L}$ & 7.3 & 316.73 & $N$ & 316.73 \\
\hline
\end{tabular}

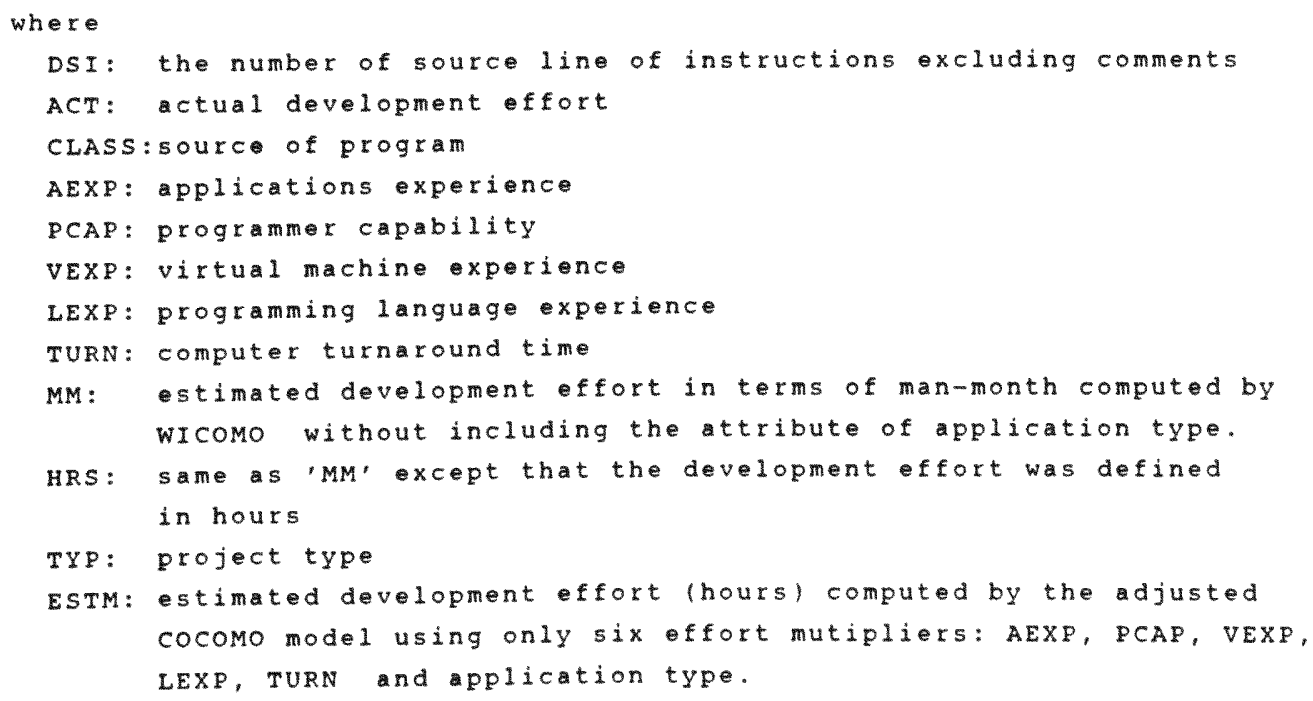


VL: very low

L: $10 \mathrm{~W}$

N: nominal

H: high

vH: very high 
Table 12. Complexity metrics of 324 NCR programs

\begin{tabular}{|c|c|c|c|c|c|c|c|}
\hline NBR & DSI & SLOC & $n$ & $N$ & $\mathrm{v}$ & $v(G)$ & $D S I / V(G)$ \\
\hline 1. & 603. & 833. & 334. & 3107. & 26048.17 & 87. & 6.93 \\
\hline 2 . & 314. & 376. & 298. & 1633. & 13421.90 & 41. & 7.66 \\
\hline 3. & 1434. & 1877. & 805. & 7727. & 74587.53 & 206 . & 6.96 \\
\hline 4. & 22. & 43 & 48 & 107. & 597.59 & 2. & 11.00 \\
\hline 5. & 104. & 141. & 128. & 600 . & 4200.00 & 12. & 8.67 \\
\hline 6. & 259. & 301 . & 181. & 1545. & 11587.26 & 45 & 5.76 \\
\hline 7. & 790 & 1155. & 312 . & 3638 . & 30142.29 & 144 . & 5.49 \\
\hline 8. & 50 & 73 & 80 & 260 & 1643.70 & 3. & 16.67 \\
\hline 9. & 387. & 592. & 231. & 1613. & 12664.87 & 40 & 9.67 \\
\hline 10. & 241. & 491. & 160. & 948. & $6941 \times 19$ & 6. & 40.17 \\
\hline 11. & 1659. & 2177 & 553. & 7653. & 69727.52 & 193. & 8.60 \\
\hline 12. & 183 & 190 & 127. & 1004. & 7016.64 & 36. & 5.08 \\
\hline 13. & 249. & 289. & 153. & 1343. & 9746.67 & 46. & 5.41 \\
\hline 14. & 572. & 843. & 240. & 2628 . & 20779.31 & 123 & 4.65 \\
\hline 15. & 243. & 421. & 212. & 1425. & 11012.29 & 30 & 8.10 \\
\hline 16. & 1122. & 1620. & 610. & 7468 . & 69098.90 & 231. & 4.86 \\
\hline 17. & 786 & 1070 & 528. & 4304 . & 38927.07 & 105. & 7.49 \\
\hline 18. & 59. & 81. & 86 & 261 & 1677.26 & 4. & 14.75 \\
\hline 19. & 32. & 51. & 52. & 152 . & 866.47 & 5. & 6.40 \\
\hline 20 & 1172 & 1837. & 670. & 7444 . & 69884.40 & 230. & 5.10 \\
\hline 21. & 39. & 60. & 64. & 163. & 978.00 & 3. & 13.00 \\
\hline 22. & 336. & 458 . & 161. & 1755. & 12865.76 & 60. & 5.60 \\
\hline 23. & 1482 . & 1725. & 599. & 6794. & 62684.24 & 191. & 7.76 \\
\hline 24. & 1038. & 1248 & 631. & 4777. & 44433.25 & 75. & 13.84 \\
\hline 25 & 841. & 1072 & 569. & 4024 . & 36828.79 & 64. & 13.14 \\
\hline 26. & 100 & 159. & 100 & 571. & 3793.64 & 12. & 8.33 \\
\hline 27 & 65. & 72. & 84. & 305. & 1949.66 & 7. & 9.29 \\
\hline 28 & 83 & 99. & 97. & 376. & 2481.57 & 9. & 9.22 \\
\hline 29. & 161. & 169. & 127. & 805. & 5625.89 & 26. & 6.19 \\
\hline 30. & 635. & 899. & 375. & 3247. & 27764.27 & 77. & 8.25 \\
\hline 31. & 315. & 415. & 246. & 1512. & 12009.08 & 17. & 18.53 \\
\hline 32. & 713. & 861. & 426. & 3785 . & 33060.88 & 69. & 10.33 \\
\hline 33. & 76. & 141. & 69. & 336. & 2052.46 & 10. & 7.60 \\
\hline 34. & 152 . & 223. & 141. & 718. & 5126.20 & 7. & 21.71 \\
\hline 35. & 160. & 212. & 154. & 921. & 6692.71 & 20. & 8.00 \\
\hline 36. & 60 & 101. & 67. & 223 & 1352.74 & 9. & 6.67 \\
\hline 37. & 1213 & 1491 & 654. & 5851. & 54725.26 & 207. & 5.86 \\
\hline 38. & 113. & 172. & 93. & 508 & 3321.89 & 31. & 3.65 \\
\hline 39. & 34. & 85. & 61. & 138. & 818.44 & 3. & 11.33 \\
\hline 40 & 93. & 132. & 99. & 384. & 2545.67 & 12. & 7.75 \\
\hline 41. & 175. & 241. & 173. & 895. & 6653.99 & 22. & 7.95 \\
\hline 42. & 227 & 294. & 193. & 1131. & 8587.07 & 32. & 7.09 \\
\hline 43. & 34. & 96. & 61. & 138 & 818.44 & 3 & 11.33 \\
\hline 44. & 107. & 184. & 109. & 582. & 3939.08 & 12 . & 8.92 \\
\hline 45 & 119 & 170. & 85. & 564. & 3614.90 & 16. & 7.44 \\
\hline 46. & 40 & 64. & 64. & 181. & 1086.00 & 7. & 5.71 \\
\hline 47 & 40. & 80. & 57. & 182. & 1061.59 & 5. & 8.00 \\
\hline 48. & 28. & 44. & 57. & 137. & 799.11 & 2. & 14.00 \\
\hline
\end{tabular}




\begin{tabular}{|c|c|c|c|c|c|c|c|}
\hline 49. & 32. & 48. & 65. & 152. & 915.40 & 3. & 10.67 \\
\hline 50. & 64. & 70. & 73. & 324. & 2005.50 & 8. & 8.00 \\
\hline 51. & 112. & 164. & 114. & 574. & 3922.08 & 15. & 7.47 \\
\hline 52. & 125. & 181. & 105. & 668. & 4485.12 & 17. & 7.35 \\
\hline 53. & 76 . & 115. & 95. & 376. & 2470.27 & 6. & 12.67 \\
\hline 54. & 438. & 606. & 163. & 2157. & 15851.21 & 72. & 6.08 \\
\hline 55. & 160. & 220 & 121. & 825. & 5708.06 & 23. & 6.96 \\
\hline 56. & 157. & 239 & 113. & 769. & 5244.72 & 28. & 5.61 \\
\hline 57. & 374. & 577 & 288 & 1807. & 14763.05 & 30. & 12.47 \\
\hline 58. & 112. & 168. & 108. & 605 & 4086.71 & 17. & 6.59 \\
\hline 59. & 624 & 892. & 317. & 3281 . & 27259.66 & 76. & 8.21 \\
\hline 60. & 128. & 188. & 115. & 675 . & 4620.71 & 20. & 6.40 \\
\hline 61. & 50. & 93. & 79 & 266. & 1676.81 & 6. & 8.33 \\
\hline 62. & 122. & 162. & 100. & 618. & 4105.90 & 16. & 7.63 \\
\hline 63. & 530. & 688. & 472. & 2626 . & 23325.82 & 29. & 18.28 \\
\hline 64. & 223 & 474 & 203. & 1122. & 8600.51 & 15. & 14.87 \\
\hline 65. & 314. & 418 & 290. & 1551. & 12687.04 & 30. & 10.47 \\
\hline 66. & 583 & 844 & 505. & 2926 . & 26275.89 & 31. & 18.81 \\
\hline 67. & 485 & 664 & 416. & 2366. & 20585.24 & 23. & 21.09 \\
\hline 68. & 370. & 478 & 284 & 1711. & 13944.22 & 23. & 16.09 \\
\hline 69. & 89. & 147. & 88. & 447 & 2887.37 & 15. & 5.93 \\
\hline 70. & 189. & 299. & 141. & 1020. & 7282.34 & 22 . & 8.59 \\
\hline 71. & 96. & 179. & 113. & 492 . & 3355.53 & 3. & 32.00 \\
\hline 72. & 123. & 180. & 110 & 659. & 4468.92 & 15. & 8.20 \\
\hline 73. & 32. & 39. & 52. & 145. & 826.56 & 1. & 32.00 \\
\hline 74. & 113. & 172. & 93. & 493. & 3223.81 & 12. & 9.42 \\
\hline 75. & 236 & 485 & 131. & 921. & 6477.78 & 4. & 59.00 \\
\hline 76. & 57. & 101. & 97. & 307. & 2026.17 & 5. & 11.40 \\
\hline 77. & 56. & 96. & 94. & 290. & 1900.83 & 5. & 11.20 \\
\hline 78. & 59. & 104. & 97. & 314. & 2072.37 & 6. & 9.83 \\
\hline 79. & 56. & 100 . & 94. & 290. & 1900.83 & 5. & 11.20 \\
\hline 80 & 78 & 128. & 97. & 363. & 2395.77 & 6. & 13.00 \\
\hline 81. & 301. & 441. & 157. & 1661. & 12116.36 & 47. & 6.40 \\
\hline 82. & 47. & 89. & 69. & 196. & 1197.27 & 3 & 15.67 \\
\hline 83. & 422 & 612. & 261. & 1894. & 15204.85 & 56. & 7.54 \\
\hline 84. & 144. & 198. & 117. & 757. & 5200.87 & 22. & 6.55 \\
\hline 85. & 293. & 432. & 339. & 1525 . & 12817.84 & 8 & 36.62 \\
\hline 86. & 431. & 618. & 403. & 2151 & 18616.12 & 29. & 14.86 \\
\hline 87. & 108. & 169. & 133. & 637. & 4494.21 & 13 & 8.31 \\
\hline 88 & 165. & 230. & 135. & 849 & 6008.22 & 24. & 6.88 \\
\hline 89. & 101. & 120. & 114. & 644. & 4400.38 & 17. & 5.94 \\
\hline 90. & 341. & 423. & 150. & 1658. & 11985.38 & 54 & 6.31 \\
\hline 91. & 237. & 262 . & 208. & 1557. & 11989.58 & 31. & 7.65 \\
\hline 92. & 202. & 222 & 205. & 1375. & 10559.29 & 22. & 9.18 \\
\hline 93. & 491. & 705. & 439. & 2543. & 22322.65 & 28. & 17.54 \\
\hline 94. & 816. & 985. & 360 & 3993. & 33907.97 & 96. & 8.50 \\
\hline 95. & 173. & 220. & 138. & 819. & 5821.88 & 29. & 5.97 \\
\hline 96. & 88. & 125. & 114. & 444 & 3033.80 & 10. & 8.80 \\
\hline 97. & 63. & 110. & 91. & 309. & 2010.91 & 5. & 12.60 \\
\hline 98. & 104. & 142. & 129. & 548 & 3842.15 & 11. & 9.45 \\
\hline 99. & 238 & 379. & 186. & 1213. & 9145.00 & 35. & 6.80 \\
\hline 100. & 57. & 102. & 97. & 307. & 2026.17 & 5. & 11.40 \\
\hline 101. & 58. & 98. & 94. & 290. & 1900.83 & 5. & 11.60 \\
\hline 102. & 137. & 237. & 112. & 776. & 5282.51 & 28. & 4.89 \\
\hline
\end{tabular}




\begin{tabular}{|c|c|c|c|c|c|c|c|}
\hline 103. & 78. & 125. & 115. & 422. & 2888.80 & 6. & 13.00 \\
\hline 104. & 56. & 100 & 94. & 290. & 1900.83 & 5. & 11.20 \\
\hline 105. & 124. & 191. & 106. & 605. & 4070.39 & 20 & 6.20 \\
\hline 106. & 20. & 74. & 44. & 92. & 502.27 & 2. & 10.00 \\
\hline 107. & 83. & 102. & 95. & 394. & 2588.52 & 13. & 6.38 \\
\hline 108. & 307. & 441. & 215. & 1541. & 11939.96 & 40 & 7.67 \\
\hline 109. & 48 & 90. & 73. & 213. & 1318.43 & 3. & 16.00 \\
\hline 110. & 98. & 146 & 128. & 565. & 3955.00 & 5. & 19.60 \\
\hline 111. & 478 & 675. & 386. & 2381. & 20458.64 & 45. & 10.62 \\
\hline 112. & 134. & 211. & 106. & 705 & 4743.18 & 18. & 7.44 \\
\hline 113. & 140. & 216. & 118. & 774. & 5327.17 & 21. & 6.67 \\
\hline 114. & 160. & 241 & 110. & 830. & 5628.53 & 25. & 6.40 \\
\hline 115. & 100. & 171. & 107. & 551. & 3714.55 & 11. & 9.09 \\
\hline 116. & 1057. & 1483 & 587. & 5800 & 53343.86 & 148. & 7.14 \\
\hline 117. & 77. & 117. & 94. & 375. & 2457.97 & 10. & 7.70 \\
\hline 118. & 354. & 487. & 293. & 1726. & 14144.15 & 45. & 7.87 \\
\hline 119. & 142. & 192. & 129. & 757 & 5307.50 & 22. & 6.45 \\
\hline 120. & 55. & 74 & 87. & 301. & 1939.33 & 4. & 13.75 \\
\hline 121. & 401. & 560. & 330. & 2420 & 20246.50 & 72. & 5.57 \\
\hline 122. & 463. & 667. & 411. & 2297 & 19944.84 & 24. & 19.29 \\
\hline 123. & 262. & 315. & 191. & 1240. & 9396.01 & 39. & 6.72 \\
\hline 124. & 105. & 160. & 95. & 508 & 3337.49 & 13. & 8.08 \\
\hline 125. & 222 & 320. & 140. & 1084. & 7728.14 & 33. & 6.73 \\
\hline 126. & 289. & 296. & 193. & 1496. & 11358.32 & 41. & 7.05 \\
\hline 127. & 290. & 311. & 190. & 1533. & 11604.59 & 43. & 6.74 \\
\hline 128. & 386. & 569. & 273. & 1836 & 14858.30 & 40. & 9.65 \\
\hline 129. & 116 & 133. & 119. & 552. & 3805.94 & 12. & 9.67 \\
\hline 130. & 116. & 134. & 113. & 544. & 3710.18 & 13. & 8.92 \\
\hline 131. & 445. & 587 & 307. & 2135. & 17639.57 & 35. & 12.71 \\
\hline 132. & 211. & 329. & 277. & 1029. & 8349.04 & 4. & 52.75 \\
\hline 133. & 113. & 164. & 129. & 542. & 3800.09 & 10. & 11.30 \\
\hline 134. & 130. & 199. & 116. & 694. & 4759.44 & 16. & 8.13 \\
\hline 135. & 592. & 858. & 312. & 2840 & 23530.54 & 69. & 8.58 \\
\hline 136. & 251. & 500. & 153. & 1056 . & 7663.80 & 8. & 31.37 \\
\hline 137. & 955. & 1321. & 584. & 4526 & 41593.15 & 80. & 11.94 \\
\hline 138. & 1528 . & 2153 & 703. & 6881. & 65076.24 & 156. & 9.79 \\
\hline 139. & 51. & 62. & 74. & 257. & 1595.83 & 7. & 7.29 \\
\hline 140. & 98. & 160. & 104. & 450 & 3015.20 & 8. & 12.25 \\
\hline 141. & 588 & 870 & 341. & 2766 . & 23272.09 & 64. & 9.19 \\
\hline 142. & 1434. & 1959. & 657. & 6368 & 59602.88 & 148. & 9.69 \\
\hline 143. & 609 & 874. & 374. & 2889. & 24691.98 & 54. & 11.28 \\
\hline 144 & 98. & 157. & 111. & 455. & 3091.46 & 10 & 9.80 \\
\hline 145. & 19. & 36. & 44. & 88 & 480.43 & 2 & 9.50 \\
\hline 146. & 29. & 44. & 48. & 129. & 720.46 & 4. & 7.25 \\
\hline 147. & 29. & 49. & 55. & 129. & 745.80 & 3. & 9.67 \\
\hline 148. & 130. & 213 & 112. & 658. & 4479.24 & 18. & 7.22 \\
\hline 149. & 32. & 45 & 54 & 164. & 943.80 & 6. & 5.33 \\
\hline 150. & 1426. & 1999. & 1025. & 8724. & 87252.28 & 49. & 29.10 \\
\hline 151. & 66. & 144. & 61. & 292. & 1731.78 & 7. & 9.43 \\
\hline 152. & 71. & 77. & 78. & 323. & 2030.18 & 9. & 7.89 \\
\hline 153. & 36. & 82. & 63. & 153. & 914.52 & 3. & 12.00 \\
\hline 154. & 34 & 93. & 57. & 156. & 909.93 & 2 & 17.00 \\
\hline 155. & 315. & 421. & 152. & 1604. & 11625.68 & 49. & 6.43 \\
\hline 156. & 112 & 175. & 121. & 527. & 3646.24 & 12. & 9.33 \\
\hline
\end{tabular}




\begin{tabular}{|c|c|c|c|c|c|c|c|}
\hline 157. & 730. & 1088. & 466. & 3337 & 29579.79 & 54. & 13.52 \\
\hline 158. & 1131. & 1763. & 659. & 5402 . & 50585.05 & 91. & 12.43 \\
\hline 159. & 674. & 982. & 498 & 3281. & 29397.77 & 50. & 13.48 \\
\hline 160. & 617. & 819. & 366 . & 3112. & 26500.86 & 73. & 8.45 \\
\hline 161. & 20. & 40. & 41. & 86. & 460.75 & 2. & 10.00 \\
\hline 162. & 703. & 1093. & 401. & 3904. & 33759.68 & 96. & 7.32 \\
\hline 163. & 448 . & 611. & 304. & 2338 & 19283.65 & 42. & 10.67 \\
\hline 164. & 184. & 241. & 158. & 847. & 6186.30 & 18. & 10.22 \\
\hline 165. & 276. & 436. & 220 & 1424. & 11080.66 & 18. & 15.33 \\
\hline 166. & 97. & 160. & 101. & 554. & 3688.65 & 11. & 8.82 \\
\hline 167. & 40 . & 47 & 65. & 199. & 1198.45 & 7. & 5.71 \\
\hline 168. & 132. & 141. & 102 . & 625. & 4170.27 & 19. & 6.95 \\
\hline 169. & 26. & 45 & 59. & 135. & 794.16 & 4. & 6.50 \\
\hline 170. & 593. & 768. & 501. & 3145 & 28206.46 & 33. & 17.97 \\
\hline 171. & 100 & 148 & 111. & 608 & 4131.00 & 12. & 8.33 \\
\hline 172. & 88. & 106. & 104. & 519. & 3477.53 & 13. & 6.77 \\
\hline 173. & 32. & 39. & 68. & 160. & 973.99 & 3. & 10.67 \\
\hline 174. & 52 . & 67. & 84. & 216. & 1380.74 & 4. & 13.00 \\
\hline 175. & 301. & 433. & 146. & 1508 . & 10842.26 & 51. & 5.90 \\
\hline 176. & 248. & 325. & 114. & 1259. & 8602.61 & 42. & 5.90 \\
\hline 177. & 70 & 111. & 101. & 389. & 2590.04 & 6. & 11.67 \\
\hline 178. & 346. & 489. & 245 . & 2118. & 16809.80 & 52. & 6.65 \\
\hline 179. & 380. & 524. & 257. & 2309 & 18484.99 & 56. & 6.79 \\
\hline 180. & 1168. & 1639. & 591. & 5257. & 48401.27 & 153. & 7.63 \\
\hline 181. & 768 & 1108 & 572. & 3929. & 35989.13 & 55. & 13.96 \\
\hline 182. & 295. & 405. & 158. & 1543. & 11269.73 & 34. & 8.68 \\
\hline 183. & 141. & 221 & 148 & 816. & 5882.91 & 20. & 7.05 \\
\hline 184. & 246. & 497 & 162. & 1060 . & 7780.24 & 7. & 35.14 \\
\hline 185. & 390. & 541. & 340 & 1878. & 15792.84 & 13. & 30.00 \\
\hline 186. & 18. & 32. & 44. & 85. & 464.05 & 2. & 9.00 \\
\hline 187. & 50. & 65. & 76. & 238. & 1487.01 & 8. & 6.25 \\
\hline 188. & 136. & 201. & 116. & 731. & 5013.18 & 18. & 7.56 \\
\hline 189. & 265 & 516 & 183. & 1092. & 8207.14 & 4. & 66.25 \\
\hline 190. & 1716. & 2423 & 697. & 8424. & 79564.80 & 198 & 8.67 \\
\hline 191. & 78. & 97. & 85. & 407 & 2608.62 & 15. & 5.20 \\
\hline 192. & 27. & 58. & 50. & 111. & 626.47 & 1. & 27.00 \\
\hline 193. & 26. & 57. & 48 & 107 & 597.59 & 1. & 26.00 \\
\hline 194. & 955. & 1143. & 214. & 3587 . & 27768.64 & 181. & 5.28 \\
\hline 195. & 175. & 249. & 116. & 912. & 6254.48 & 27. & 6.48 \\
\hline 196. & 148. & 208. & 116. & 752. & 5157.20 & 20 & 7.40 \\
\hline 197. & 126. & 192. & 101. & 673. & 4480.98 & 17. & 7.41 \\
\hline 198. & 124. & 164. & 115. & 850. & 5818.67 & 10. & 12.40 \\
\hline 199. & 36. & 53. & 62. & 203 & 1208.70 & 5. & 7.20 \\
\hline 200 & 312. & 430. & 286. & 1918. & 15650.63 & 21. & 14.86 \\
\hline 201. & 1470. & 1914. & 603. & 7842 . & 72428.82 & 215 & 6.84 \\
\hline 202 . & 623 & 741. & 290. & 4055 . & 33169.53 & 101. & 6.17 \\
\hline 203. & 1637. & 2280 . & 621. & 8074 . & 74914.20 & 287. & 5.70 \\
\hline 204. & 535. & 585. & 300. & 2995. & 24645.31 & 112. & 4.78 \\
\hline 205. & 138 & 196. & 139. & 694. & 4940.55 & 18. & 7.67 \\
\hline 206. & 305. & 367. & 219. & 1608. & 12501.86 & 52. & 5.87 \\
\hline 207. & 558. & 656. & 280. & 3120. & 25363.36 & 121. & 4.61 \\
\hline 208. & 424 & 491. & 256. & 2539. & 20312.00 & 91. & 4.66 \\
\hline 209. & 533. & 640. & 291. & 3104 . & 25405.85 & 121. & 4.40 \\
\hline 210. & 285 & 348 . & 200. & 1534. & 11725.68 & 49. & 5.82 \\
\hline
\end{tabular}




\begin{tabular}{|c|c|c|c|c|c|c|c|}
\hline 211. & 236. & 325. & 139. & 1212. & 8628.16 & 43. & 5.49 \\
\hline 212. & 317. & 370. & 231. & 1685 . & 13230.20 & 53. & 5.98 \\
\hline 213. & 200. & 261. & 141. & 1031. & 7360.88 & 33. & 6.06 \\
\hline 214. & 247. & 338. & 177. & 1221. & 9117.95 & 30. & 8.23 \\
\hline 215. & 126. & 160. & 153. & 658. & 4775.36 & 8. & 15.75 \\
\hline 216. & 315. & 411. & 213. & 1618. & 12514.76 & 48. & 6.56 \\
\hline 217. & 469 . & 621. & 440. & 2474 & 21725.08 & 20. & 23.45 \\
\hline 218. & 55. & 89. & 73. & 240 & 1485.56 & 4. & 13.75 \\
\hline 219. & 1106. & 1505 . & 656. & 5956. & 55733.58 & 182. & 6.08 \\
\hline 220. & 50. & 78. & 90. & 310. & 2012.47 & 1. & 50.00 \\
\hline 221 & 320. & 503. & 320. & 1912. & 15911.53 & 20. & 16.00 \\
\hline 222 . & 146. & 191. & 143. & 793. & 5677.78 & 13. & 11.23 \\
\hline 223 & 215 & 355. & 153. & 1146 & 8316.97 & 24. & 8.96 \\
\hline 224 & 137. & 256. & 119. & 626 & 4316.16 & 13. & 10.54 \\
\hline 225. & 388. & 581. & 399. & 1895. & 16373.26 & 15. & 25.87 \\
\hline 226. & 572 . & 662. & 313. & 3090 & 25616.16 & 106. & 5.40 \\
\hline 227. & 319. & 406 & 276 . & 1570 & 12730.38 & 29. & 11.00 \\
\hline 228 & 109. & 160. & 106. & 601. & 4043.48 & 13. & 8.38 \\
\hline 229. & 41. & 57. & 59. & 204 & 1200.06 & 5. & 8.20 \\
\hline 230. & 182. & 235. & 162. & 945 . & 6936.16 & 27. & 6.74 \\
\hline 231. & 94. & 139. & 102. & 483. & 3222.78 & 10. & 9.40 \\
\hline 232. & 41. & 61. & 67. & 209. & 1267.81 & 2. & 20.50 \\
\hline 233. & 152. & 200 & 116. & 748 . & 5129.77 & 25. & 6.08 \\
\hline 234. & 1173. & 1616. & 653. & 6286 & 58780.00 & 155. & 7.57 \\
\hline 235. & 1331. & 1676. & 608 . & 6638 & 61387.74 & 138. & 9.64 \\
\hline 236. & 163. & 241. & 181. & 754. & 5654.88 & 4. & 40.75 \\
\hline 237 & 73 & 98. & 97. & 342. & 2257.17 & 6. & 12.17 \\
\hline 238. & 244 . & 495. & 157. & 931. & 6791.29 & 5. & 48.80 \\
\hline 239. & 711 & 936. & 345 . & 4040 & 34059.03 & 118. & 6.03 \\
\hline 240 & 43 & 50. & 69. & 196. & 1197.27 & 3. & 14.33 \\
\hline 241. & 1571. & 2163 & 771. & 7312. & 70126.37 & 213. & 7.38 \\
\hline 242 . & 85. & 179. & 114. & 535. & 3655.60 & 10. & 8.50 \\
\hline 243 & 979. & 1591. & 649. & 6027. & 56304.68 & 118. & 8.30 \\
\hline 244. & 30. & 51. & 58. & 121. & 708.82 & 1. & 30.00 \\
\hline 245 . & 171. & 272 . & 139. & 892. & 6350.10 & 24. & 7.13 \\
\hline 246. & 26. & 69. & 59. & 120. & 705.92 & 3. & 8.67 \\
\hline 247. & 47. & 65. & 74. & 197. & 1223.26 & 2. & 23.50 \\
\hline 248. & 68. & 108. & 83. & 327. & 2084.64 & 8. & 8.50 \\
\hline 249. & 1061. & 1500 . & 561. & 5496. & 50188.69 & 100. & 10.61 \\
\hline 250. & 139. & 189. & 128. & 704 . & 4928.00 & 10. & 13.90 \\
\hline 251. & 52. & 88. & 87. & 247 & 1591.41 & 6. & 8.67 \\
\hline 252. & 52. & 97. & 87. & 247 & 1591.41 & 6. & 8.67 \\
\hline 253. & 177. & 295. & 129. & 968. & 6786.87 & 30. & 5.90 \\
\hline 254. & 270 & 387. & 231. & 1370. & 10756.90 & 15. & 18.00 \\
\hline 255 . & 52. & 70. & 77. & 268 & 1679.50 & 4. & 13.00 \\
\hline 256. & 72. & 113. & 102. & 395. & 2635.61 & 6. & 12.00 \\
\hline 257. & 400 & 522. & 220 & 1994. & 15516.03 & 58. & 6.90 \\
\hline 258. & 510. & 684. & 259. & 2487 & 19937.80 & 55. & 9.27 \\
\hline 259. & 93. & 136. & 95. & 487 & 3199.52 & 10. & 9.30 \\
\hline 260. & 77. & 133. & 86. & 415 & 2666.90 & 14. & 5.50 \\
\hline 261. & 148. & 214. & 100. & 736 & 4889.88 & 21. & 7.05 \\
\hline 262. & 347 . & 499. & 320. & 1806 & 15029.40 & 28. & 12.39 \\
\hline 263. & 3631. & 4960. & 1186. & 17019. & 173796.12 & 449. & 8.09 \\
\hline 64. & 432 & 592. & 237. & 2203 & 17378.90 & 62. & 6.97 \\
\hline
\end{tabular}




\begin{tabular}{|c|c|c|c|c|c|c|c|}
\hline 265 & 100. & 144. & 110. & 535. & 3628.03 & 12. & 8.33 \\
\hline 266. & 126. & 179. & 107. & 690. & 4651.61 & 16. & 7.88 \\
\hline 267. & 42. & 78. & 78. & 224. & 1407.93 & 5. & 8.40 \\
\hline 268. & 256. & 506. & 164. & 970. & 7136.83 & 5. & 51.20 \\
\hline 269. & 1770. & 2427 & 513. & 8023. & 72229.58 & 248. & 7.14 \\
\hline 270. & 1928 & 2252 . & 573 & 8753. & 80198.41 & 292. & 6.60 \\
\hline 271. & 196. & 345 & 184. & 943. & 7094.72 & 10 & 19.60 \\
\hline 272. & 125. & 172. & 100 & 616. & 4092.62 & 17. & 7.35 \\
\hline 273. & 81. & 128 & 102. & 391. & 2608.92 & 7. & 11.57 \\
\hline 274 & 119. & 165. & 111. & 597. & 4056.27 & 16. & 7.44 \\
\hline 275 . & 137. & 225 & 133. & 723. & 5100.97 & 6. & 22.83 \\
\hline 276 & 30. & 74. & 55. & 147 & 849.86 & 1. & 30.00 \\
\hline 277. & 96. & 137. & 117. & 422 & 2899.29 & 8. & 12.00 \\
\hline 278 . & 118. & 151. & 124. & 589. & 4096.02 & 15. & 7.87 \\
\hline 279. & 89. & 115. & 105 & 433 & 2907.27 & 12. & 7.42 \\
\hline 280 & 97. & 134. & 73 & 467 & 2890.65 & 22. & 4.41 \\
\hline 281. & 92. & 107. & 96. & 468 & 3081.76 & 13. & 7.08 \\
\hline 282. & 93. & 109. & 99. & 485 & 3215.24 & 12. & 7.75 \\
\hline 283. & 272 & 399. & 187. & 1292. & 9750.59 & 35. & 7.77 \\
\hline 284 & 450. & 543. & 257. & 2499. & 20006.06 & 88. & 5.11 \\
\hline 285 . & 321. & 459. & 235. & 1928. & 15185.92 & 35. & 9.17 \\
\hline 286. & 1204. & 1744. & 748 & 6915. & 66016.77 & 168. & 7.17 \\
\hline 287. & 57. & 106. & 97. & 345. & 2276.97 & 4. & 14.25 \\
\hline 288. & 46. & 113. & 72 . & 222. & 1369.72 & 4. & 11.50 \\
\hline 289. & 27. & 44. & 56. & 124. & 720.11 & 1. & 27.00 \\
\hline 290. & 373. & 481. & 365. & 2106 & 17925.75 & 19. & 19.63 \\
\hline 291. & 217. & 302 . & 137. & 1210. & 8588.62 & 20. & 10.85 \\
\hline 292. & 191. & 264. & 193. & 2248 & 17067.84 & 18. & 10.61 \\
\hline 293. & 577. & 801. & 483 & 2726. & 24304.69 & 35. & 16.49 \\
\hline 294. & 1277. & 1874. & 659. & 6375. & 59696.36 & 155 . & 8.24 \\
\hline 295. & 486. & 689. & 232. & 2381. & 18709.85 & 52. & 9.35 \\
\hline 296. & 284 & 412. & 233 & 1756. & 13809.51 & 34. & 8.35 \\
\hline 297. & 208. & 400 & 154. & 1052. & 7644.66 & 32. & 6.50 \\
\hline 298. & 1044 & 1624. & 616. & 5466 . & 50652.25 & 75. & 13.92 \\
\hline 299. & 554. & 733. & 757 & 2448 & 23413.04 & 25 . & 22.16 \\
\hline 300 & 656. & 814. & 808 & 3141. & 30336.44 & 35. & 18.74 \\
\hline 301. & 430. & 687. & 346 & 2058 . & 17358.46 & 23. & 18.70 \\
\hline 302. & 1502. & 2586. & 599. & 8472 . & 78166.16 & 213. & 7.05 \\
\hline 303. & 522 . & 696. & 417. & 2925. & 25458.92 & 79. & 6.61 \\
\hline 304. & 1040 & 1568. & 549. & 5123. & 46622.69 & 110. & 9.45 \\
\hline 305. & 91. & 118. & 102. & 420 & 2802.42 & 9. & 10.11 \\
\hline 306. & 2796 & 4238 . & 1069. & 15028. & 151212.43 & 347. & 8.06 \\
\hline 307. & 356. & 411. & 291. & 1741. & 14249.87 & 29. & 12.28 \\
\hline 308. & 442 & 625 . & 530 & 2157 & 19520.52 & 25. & 17.68 \\
\hline 309. & 920. & 1021. & 866. & 4057. & 39589.11 & 70. & 13.14 \\
\hline 310. & 434. & 648. & 455 & 1993. & 17597.64 & 27. & 16.07 \\
\hline 311. & 967. & 1067. & 891. & 4375. & 42871.86 & 66. & 14.65 \\
\hline 312. & 35. & 51. & 60. & 197. & 1163.66 & 4. & 8.75 \\
\hline 313. & 1685. & 2231. & 1249 & 8107. & 83393.12 & 143. & 11.78 \\
\hline 314. & 95. & 145. & 118 & 490. & 3372.50 & 8. & 11.88 \\
\hline 315. & 315. & 633. & 197. & 1827. & 13925.49 & 20 & 15.75 \\
\hline 316. & 363. & 445 . & 294. & 2372 . & 19449.62 & 25. & 14.52 \\
\hline 317. & 1164. & 1614. & 415 & 5460 . & 47485.44 & 148. & 7.86 \\
\hline 318. & 680. & 1256. & 474. & 3656 . & 32497.24 & 44. & 15.45 \\
\hline
\end{tabular}




$\begin{array}{rrrrrrrr}319 . & 102 . & 207 . & 102 . & 653 . & 4357.09 & 9 . & 11.33 \\ 320 . & 773 . & 1190 . & 526 . & 4029, & 36417.80 & 63 . & 12.27 \\ 321 . & 827 . & 1209 . & 387 . & 4557 . & 39172.84 & 106 . & 7.80 \\ 322 . & 605 . & 824 . & 449 . & 3312 . & 29180.61 & 55 . & 11.00 \\ 323 . & 175 . & 286 . & 169 . & 1113 . & 8237.18 & 17 . & 10.29 \\ 324 . & 260 . & 329 . & 263 . & 2792 . & 22444.66 & 48 . & 5.42\end{array}$

wher

DSI: the number of source line of instructions excluding comments

SLOC: the number of source lines including comments.

n: Halstod's vocabulary (i.e. total number of distinct operators and operands)

N: Halsted's program length (i.e. total number of occurrences of operators and operands)

V: Halstead's program volume

$V(G)$ : McCabe's cyclomatic complexity (i. . basic program control paths) which is oqual to the number of preaicates plus one

DSI/V(G): the number of sequential instructions per V(G) 
Tabl. 13. Actul Development Efort and calculated Model Development Efforts for 28 IU Projects

\begin{tabular}{|c|c|c|c|c|c|c|c|c|c|}
\hline $\mathrm{Nb} \mathrm{x}$ & DSI & SLOC & $\begin{array}{l}\mathrm{ACT} \\
\mathrm{EFF}\end{array}$ & $\begin{array}{c}\text { HLSTD } \\
\text { EFF }\end{array}$ & $\begin{array}{l}\text { IBM } \\
\text { EFF }\end{array}$ & $\begin{array}{l}\text { SLIM } \\
\text { EFF }\end{array}$ & $\begin{array}{l}\text { COCOMO } \\
\text { EFF }\end{array}$ & $\begin{array}{c}\text { ADJST } \\
\text { COCOMOEFF }\end{array}$ & CLASS \\
\hline 1 & 180 & 304 & 20 & 5.74 & 76.25 & 23.55 & 13.00 & 18.20 & DS . KK \\
\hline 2 & 180 & 309 & 9 & 5.59 & 77.39 & 22.50 & 13.00 & 18.20 & DS.LF \\
\hline 3 & 194 & 261 & 4 & 5.58 & 66.37 & 10.00 & 4.33 & 13.00 & MACKY \\
\hline 4 & 224 & 322 & 30 & 7.25 & 80.35 & 37.98 & 8.67 & 28.17 & LOOK \\
\hline 5 & 248 & 350 & 15 & 16.66 & 86.68 & 37.50 & 17.33 & 24.27 & $D S \cdot K B$ \\
\hline 6 & 296 & 382 & 18 & 12.13 & 93.86 & 37.69 & 21.67 & 30.33 & DS. LG \\
\hline 7 & 316 & 524 & 22 & 13.71 & 125.15 & 46.09 & 21.67 & 56.33 & $D S . \angle B$ \\
\hline 8 & 384 & 533 & 80 & 13.46 & 127.10 & 152.32 & 17.33 & 61.97 & OS.BK \\
\hline 9 & 392 & 679 & 40 & 18.41 & 158.43 & 64.41 & 17.33 & 56.33 & OS.LI \\
\hline 10 & 473 & 907 & 80 & 23.16 & 206.18 & 126.91 & 21.67 & 67.60 & SFENG \\
\hline 11 & 480 & 592 & 50 & 40.28 & 139.84 & 108.64 & 17.33 & 30.33 & UPDAT \\
\hline 12 & 535 & 668 & 30 & 16.13 & 156.09 & 64.69 & 13.00 & 30.33 & PRABU \\
\hline 13 & 547 & 637 & 20 & 27.46 & 149.48 & 50.00 & 60.67 & 75.83 & PASCL \\
\hline 14 & 550 & 977 & 40 & 20.39 & 220.61 & 94.20 & 26.00 & 84.50 & OS.M \\
\hline 15 & 593 & 917 & 90 & 37.09 & 208.25 & 156.12 & 43.33 & 112.67 & SPELL \\
\hline 16 & 658 & 1069 & 40 & 66.17 & 239.44 & 71.92 & 47.67 & 66.73 & $O R G Z N$ \\
\hline 17 & 677 & 1083 & 90 & 53.54 & 242.29 & 123.01 & 39.00 & 112.67 & OS.LW \\
\hline 18 & 720 & 841 & 60 & 67.41 & 192.48 & 78.83 & 17.33 & 39.43 & PRABU \\
\hline 19 & 740 & 987 & 60 & 74.86 & 222.67 & 85.59 & 21.67 & 58.93 & MTRXL \\
\hline 20 & 823 & 1077 & 80 & 83.51 & 241.07 & 157.82 & 39.00 & 77.35 & MTRXF \\
\hline 21 & 879 & 1566 & 70 & 60.18 & 338.91 & 173.68 & 39.00 & 129.57 & OS.HL \\
\hline 22 & 1139 & 1471 & 60 & 89.48 & 320.15 & 73.68 & 30.33 & 66.73 & PRABU \\
\hline 23 & 1311 & 1579 & 160 & 120.77 & 341.47 & 378.82 & 52.00 & 169.00 & REVEW \\
\hline 24 & 1653 & 2579 & 200 & 222.54 & 533.64 & 311.03 & 78.00 & 190.67 & PASER \\
\hline 25 & 1786 & 3734 & 225 & 202.81 & 747.32 & 462.54 & 47.67 & 151.67 & $N C R C N$ \\
\hline 26 & 1821 & 2332 & 250 & $223 \cdot 31$ & 486.92 & 321.66 & 86.67 & 212.33 & CMP.L \\
\hline 27 & 2165 & 3742 & 275 & 222.35 & 748.77 & 369.21 & 104.00 & 251.33 & STEVE \\
\hline 28 & 2683 & 4068 & 320 & 345.82 & 807.91 & 383.26 & 130.00 & 316.73 & CPM.C \\
\hline
\end{tabular}

where

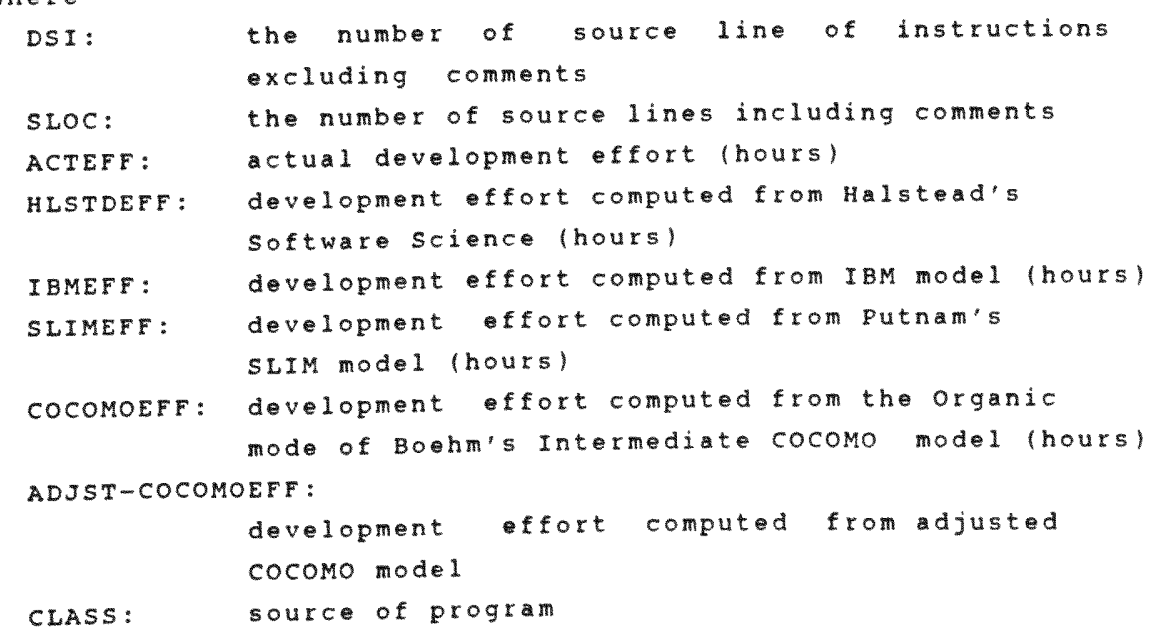


Tabl 14. Relative Errors of Models' Development Efforts of FIU Projects

\begin{tabular}{|c|c|c|c|c|c|c|c|c|c|c|}
\hline \multirow{3}{*}{ NBR } & \multirow{3}{*}{$x-Y 1$} & \multicolumn{2}{|c|}{$A B S(X-Y 1)$} & \multicolumn{2}{|c|}{$\operatorname{ABS}(X-Y 2)$} & \multicolumn{2}{|l|}{$A B S(X-Y 3)$} & \multirow[t]{2}{*}{$A B S(X-x 4)$} & \multirow{3}{*}{$X-Y 5$} & \multirow[t]{2}{*}{$\operatorname{ABS}(x-y 5)$} \\
\hline & & & $x-y 2$ & & $x-x 3$ & & $X-Y 4$ & & & \\
\hline & & $\mathrm{x}$ & & $\mathrm{x}$ & & $\mathrm{x}$ & & $x$ & & $x$ \\
\hline 1 & 14.26 & 0.71 & -56.25 & 2.81 & -3.55 & 0.18 & 7.00 & 0.35 & 1.80 & 0.09 \\
\hline 2 & 3.41 & 0.40 & -68.39 & 7.60 & -13.50 & 1.50 & -4.00 & 0.44 & -9.20 & 1.02 \\
\hline 3 & $-1 \cdot 58$ & 0.40 & -62.37 & 15.59 & -6.00 & 1.50 & -0.33 & 0.08 & -9.00 & 2.25 \\
\hline 4 & 22.75 & 0.76 & -50.35 & 1.68 & -7.98 & 0.27 & 21.33 & 0.71 & 1.83 & 0.06 \\
\hline 5 & -1.66 & 0.11 & -71.68 & 4.78 & -22.50 & 1.50 & -2.33 & 0.16 & -9.27 & 0.62 \\
\hline 6 & 5.87 & 0.33 & -75.86 & 4.21 & -19.69 & 1.09 & -3.67 & 0.20 & -12.33 & 0.69 \\
\hline 7 & 8.29 & 0.38 & -103.15 & 4.69 & -24.09 & 1.10 & 0.33 & 0.02 & -34.33 & 1.56 \\
\hline 8 & 66.54 & 0.83 & -47.10 & 0.59 & -72.32 & 0.91 & 62.67 & 0.78 & 18.03 & 0.23 \\
\hline 9 & 21.59 & 0.54 & -118.43 & 2.96 & -24.41 & 0.61 & 22.67 & 0.57 & -16.33 & 0.41 \\
\hline 10 & 56.84 & 0.71 & -126.18 & 1.58 & -46.91 & 0.59 & 58.33 & 0.73 & 12.40 & 0.16 \\
\hline 11 & 9.72 & 0.19 & -89.84 & 1.80 & -58.64 & 1.17 & 32.67 & 0.65 & 19.67 & 0.40 \\
\hline 12 & 13.87 & 0.46 & -126.09 & 4.20 & -34.69 & 1.16 & 17.00 & 0.57 & -0.33 & 0.01 \\
\hline 13 & -7.46 & 0.37 & -129.48 & 6.47 & -30.00 & 1.50 & -40.67 & 2.03 & -55.83 & 2.79 \\
\hline 14 & 19.61 & 0.49 & -180.61 & 4.52 & -54.20 & 1.36 & 14.00 & 0.35 & -44.50 & 1.11 \\
\hline 15 & 52.91 & 0.59 & $-118 \cdot 25$ & 1.31 & -66.12 & 0.74 & 46.67 & 0.52 & -22.67 & 0.25 \\
\hline 16 & -26.17 & 0.65 & -199.44 & 4.99 & -31.92 & 0.80 & -7.67 & 0.19 & -26.73 & 0.67 \\
\hline 17 & 36.46 & 0.41 & -152.29 & 1.69 & -33.01 & 0.37 & 51.00 & 0.57 & -22.67 & 0.25 \\
\hline 18 & -7.41 & 0.12 & -132.48 & 2.21 & -18.83 & 0.31 & 42.67 & 0.71 & 20.57 & 0.34 \\
\hline 19. & -14.86 & 0.25 & -162.67 & 2.71 & -25.59 & 0.43 & 38.33 & 0.64 & 1.07 & 0.02 \\
\hline 20 & -3.51 & 0.04 & -161.07 & 2.01 & -77.82 & 0.97 & 41.00 & 0.51 & 2.65 & 0.03 \\
\hline 21 & 9.82 & 0.14 & -268.91 & 3.84 & -103.68 & 1.48 & 31.00 & 0.44 & -59.57 & 0.85 \\
\hline 22 & -29.48 & 0.49 & -260.15 & 4.34 & -13.68 & 0.23 & 29.67 & 0.50 & -6.73 & 0.11 \\
\hline 23 & 39.23 & 0.25 & -181.47 & 1.13 & -218.82 & 1.37 & 108.00 & 0.68 & -9.00 & 0.06 \\
\hline 24 & -22.54 & 0.11 & -333.64 & 1.67 & -111.03 & 0.56 & 122.00 & 0.61 & 9.33 & 0.05 \\
\hline 25 & 22.19 & 0.10 & -522.32 & 2.32 & -237.54 & 1.06 & 177.33 & 0.79 & 73.33 & 0.33 \\
\hline 26 & 26.69 & 0.11 & -236.92 & 0.95 & -71.66 & 0.29 & 163.33 & 0.65 & 37.67 & 0.15 \\
\hline 27 & 52.65 & 0.19 & -473.77 & 1.72 & -94.21 & 0.34 & 171.00 & 0.62 & 23.67 & 0.09 \\
\hline 28 & -25.82 & 0.08 & -487.91 & 1.52 & $-63 \cdot 26$ & 0.20 & 190.00 & 0.59 & 3.27 & 0.01 \\
\hline
\end{tabular}

where

$X$ and $Y$ are efforts of 28 projects listed in Table 13

$x$ : represents actual development effort

Y1: represents development effort computed from halstead's software science

Y2: represents development effort computed from IBM model

Y3: represents development efort computed from putram's SLIM model

4 : represents development effort computed from the organic mode of Boehm's Intermediate cocomo model

Y5: represents development fort computed from adjusted cocomo model

ABS: represents absolute value

$A B S(X-Y 1) / X$ : xelative exror of development effort computed from

Halstead's software science

ABS $(X-Y 2), X$ : relative eror of development effort computed from IBM model

ABS $(X-Y 3) / X$; relative eror of development effort computed from 


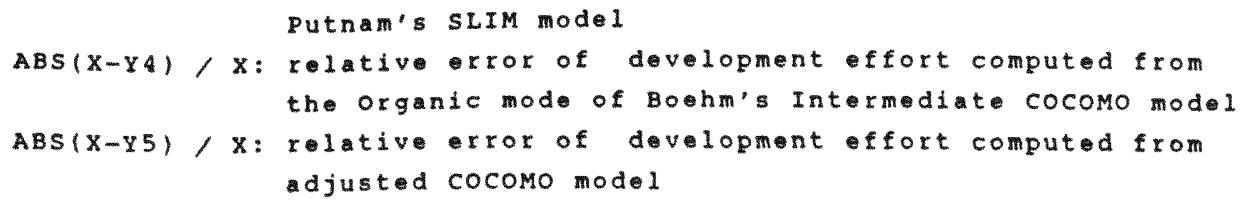

\begin{tabular}{lccr}
\hline & TOTAL & AVERAGE & RANGE OF \\
MODEL & RELATIVE ERROR & RELATIVE ERROR & RELATIVE ERROR \\
\hline HALSTEAD & 10.22 & 0.36 & $0.08--0.83$ \\
IBM & 95.89 & 3.42 & $0.59--15.59$ \\
SLIM & 23.59 & 0.84 & $0.18-1.50$ \\
COCOMO & 15.67 & 0.56 & $0.08-2.03$ \\
ADJUSTED COCOMO & 14.61 & 0.52 & $0.01--2.79$ \\
\hline
\end{tabular}

where

AVERAGE RELATIVE ERROR: the $a t i o$ of TOTAL RELATIVE ERROR Of 28 projects to 28 
Table 15. Relative Erors of Models' Dovelopment Eforts of FIU projects with program Lengths over 1000 Lines

\begin{tabular}{|c|c|c|c|c|c|c|c|c|c|c|}
\hline \multirow{3}{*}{ NBR } & \multirow{3}{*}{$X-Y 1$} & \multicolumn{2}{|c|}{$\operatorname{BS}(X-X 1)$} & \multirow[t]{2}{*}{$A B S(X-X 2)$} & \multirow{3}{*}{$x-x 3$} & \multirow[t]{2}{*}{$A B S(x-x 3)$} & \multirow{2}{*}{\multicolumn{2}{|c|}{$A B S(X-X 4)$}} & \multirow{3}{*}{$x-x 5$} & \multirow{3}{*}{$\frac{\operatorname{ABS}(x-y 5)}{x}$} \\
\hline & & & $x-y 2$ & & & & & & & \\
\hline & & $x$ & & $x$ & & $x$ & & $x$ & & \\
\hline 1 & -29.48 & 0.49 & -260.15 & 4.34 & -13.68 & 0.23 & 29.67 & 0.50 & -6.73 & 0.11 \\
\hline 2 & 39.23 & 0.25 & -181.47 & 1.13 & -218.82 & 1.37 & 108.00 & 0.68 & -9.00 & 0.05 \\
\hline 3 & $-22 \cdot 54$ & 0.11 & -333.64 & 1.67 & -111.03 & 0.56 & 122.00 & 0.61 & 9.33 & 0.05 \\
\hline 4 & 22.19 & 0.10 & -522.32 & 2.32 & -237.54 & 1.06 & 177.33 & 0.79 & 73.33 & 0.33 \\
\hline 5 & 26.69 & 0.11 & -236.92 & 0.95 & -71.66 & 0.29 & 163.33 & 0.65 & 37.67 & 0.15 \\
\hline 6 & 52.65 & 0.19 & -473.77 & 1.72 & -94.21 & 0.34 & 171.00 & 0.63 & 23.67 & 0.09 \\
\hline 7 & -25.82 & 0.09 & -487.91 & 1.52 & $-63 \cdot 26$ & 0.20 & 190.00 & 0.59 & 3.27 & 0.01 \\
\hline
\end{tabular}

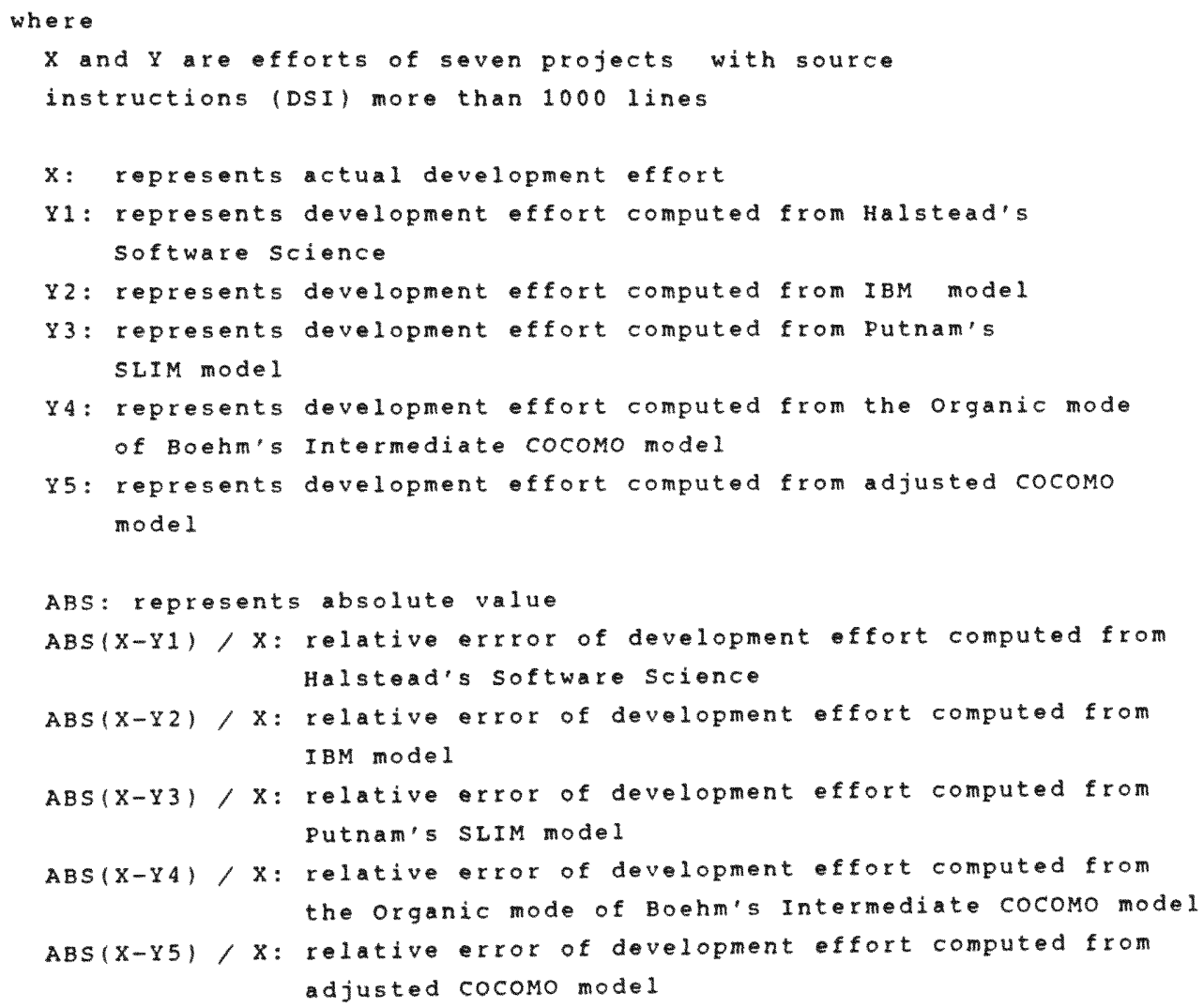


83

The average relative errors and their ranges of above seven observations of 11 models re listed below:

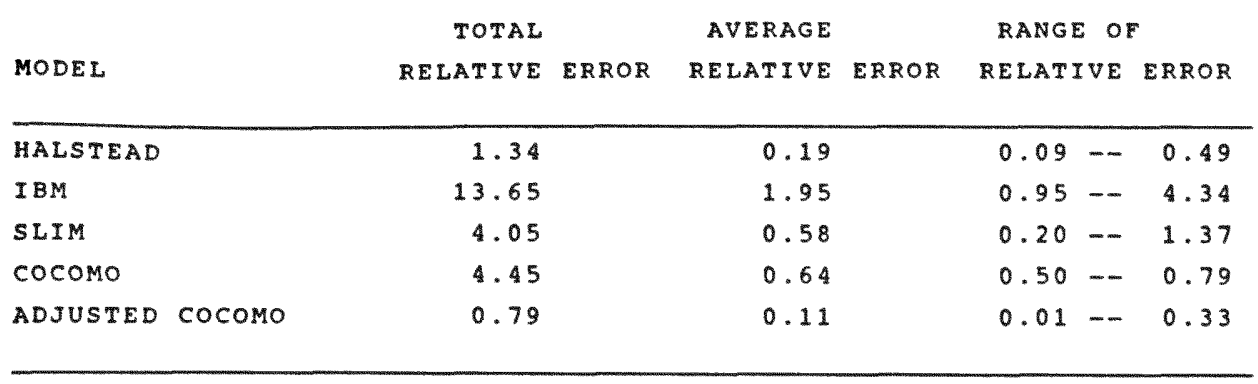

where

AVERAGE RELATIVE ERROR: the ratio of TOTAL RELATIVE ERROR Of seven projects to seven. 


\begin{tabular}{|c|c|c|c|c|c|c|c|c|c|}
\hline \multirow{2}{*}{$\begin{array}{l}\text { DEPENDENT } \\
\text { VARTABLE }\end{array}$} & \multicolumn{4}{|c|}{ INDEPENDENT } & $\mathrm{F}-\mathrm{STAT}$ & \multirow{2}{*}{$\bar{B}_{0}$} & \multirow{2}{*}{$\bar{B}_{1}$} & \multirow{2}{*}{$\begin{array}{c}\text { AVG SQRD } \\
\text { DEL RESIDUAL }\end{array}$} & \multirow[b]{2}{*}{$R^{2} p r e d$} \\
\hline & VARIABLE & $\mathrm{R}^{2}$ & $\tau$ & MSE & $(0.001$ SIG $)$ & & & & \\
\hline ACTEFF & IBMEFF & 0.8931 & 0.9451 & 849.32 & 217.25 & -16.5486 & 0.3904 & 942.99 & 0.87 \\
\hline ACTEEF & SLIMEFF & 0.8816 & 0.9389 & 941.15 & 193.52 & -3.3760 & 0.6294 & 1.232 .23 & 0.83 \\
\hline ACTEFF & COCOMOEFF & 0.7845 & 0.8857 & $1,712.22$ & 94.66 & -8.3229 & 2.5471 & 1.773 .28 & 0.76 \\
\hline ACTEFF & ADJSTEFF & 0.9056 & 0.9516 & 749.99 & 249.47 & -11.8439 & 1.0856 & 785.93 & 0.89 \\
\hline ACTEFF & HLSTDEFF & 0.9142 & 0.9561 & 682.02 & 276.92 & 16.0050 & 0.9496 & 760.67 & 0.90 \\
\hline ACTEFF & $V(G)$ & 0.9003 & 0.9489 & 791.99 & 234.86 & -3.3093 & 0.8543 & 892.06 & 0.88 \\
\hline ACTEFF & $v^{*}$ & 0.7886 & 0.8889 & 1.680 .22 & 96.96 & -8.1222 & 0.2633 & 1.766 .55 & 0.76 \\
\hline $\mathrm{n}$ & DSI & 0.9291 & 0.9638 & 3.337 .63 & 680.89 & 55.3674 & 0.3735 & $3,700.86$ & 0.92 \\
\hline$v$ & DSI & 0.9767 & 0.9883 & $26,825,643.34$ & 2.183 .07 & -7.995 .6900 & 59.9500 & $32,155,787.63$ & 0.97 \\
\hline$v(G)$ & DSI & 0.9084 & 0.9531 & 730.42 & 515.37 & -12.0897 & 0.1519 & 880.74 & 0.89 \\
\hline
\end{tabular}

where

$\mathrm{R}^{2}$ (squared correlation):

represents the proportion of the total variability of the dependent variable, x, that is accounted for by the independent variable, $x$. It is defined as

$$
R^{2}=S S R / S_{Y Z}
$$

SSR: represents regression sum of squares. It is defined as

$$
S S R=\Sigma(\tilde{Y}-\bar{Y})^{2}
$$

$S_{Y Y}$ : represents the variability in $Y$ without considering the effect of the regressor variable $X$. It is defined as

$$
s_{Y Y}=\Sigma(Y-\bar{Y})^{2}
$$

F (correlation coefficient):

$$
\text { measures the strength of the relationship between variables } X \text { and } Y \text {. It is the square root of }{ }^{2}
$$


MSE (residual mean square or mean square exror):

measures the average squared deviation between the observed and predicted values of dependent variable

F-STAT (F statistics):

It is used to test the linear relationship between variables $X$ and $Y\left(i . \theta\right.$. to test Ho: B $\left.{ }_{1}=0\right)$. It is detined as

$$
F=M S R / M S E
$$

where

MSR : represents mean square due to regression

MSE : as described above

SIG (level of significance): represents the weight of the evidence for rejecting the null hypothesis, Ho. This probability is sometimes designated by the letter P. For example, in 1 inear regression, if p< 0.001 sIG, it means that when Ho is true (i.e. no linear relationship), the probability of rejecting Ho is less than $1 / 1000$ given the sample results. In other words there is a very little chance of saying that there is no linear relationship between $X$ and $Y$.

$\tilde{B}_{0}$ : represents the estimte of intercept of the regression line. It is defined as

$$
\tilde{\mathrm{B}}_{0}=\overline{\mathrm{y}}-\tilde{\mathrm{B}}_{1} \overline{\mathrm{X}}
$$

$\tilde{B}_{1}$ : estimates the predicted change in $Y$ for a unit change in $X$. The equation is defined as

$$
\tilde{\mathrm{B}}_{1}=\mathrm{s}_{\mathrm{XX}} / \mathrm{s}_{\mathrm{XX}}
$$

where

$$
\begin{aligned}
& s_{X X}=\Sigma(X)^{2}-\left((\Sigma X)^{2} / n\right) \\
& s_{X Y}=\Sigma(X * Y)-(((\Sigma X)(\Sigma X)) / n)
\end{aligned}
$$

AVG SQRD DEL RESIDUAL:

represents verage squared deleted residual. It is used to calculate press statistics described earlier in section 5.1 .4 .

$R^{2}$ pred: represents the predicted squared multiple correlation calculating from 1 - (PRESS/ SYY) 
IBMEFF: development effort computed from IBM model

SLIMEFF: development effort computed from putnam's SLIM model

COCOMOEFF: development fort computed from the organic mode of Boehm's Intermediate cocomo model

ADJSTEFF: development efort computed from adjusted cocomo model

HLSTDEFF: development effort computed from Halstead's software science

DSI: the number of source line of instructions excluding coments

n: Halstead's vocabulary (i.e. total number of occurrences of operators and operands)

V: Halstead's program volume

$V^{*}$ : Halstead's potential volume of an algorithm

$V(G)$ : Mccabe's cyclomatic complexity (i.e. basic program control paths) which is equal to the number of predicates plus one 
Table 17. Nonlinear Rogression Results of FIU Projects

\begin{tabular}{llccrc}
\hline \multicolumn{7}{l}{$\begin{array}{l}\text { DEPENDENT } \\
\text { VARIABLE }\end{array}$} & $\begin{array}{l}\text { INDEPENDENT } \\
\text { VARIABLE }\end{array}$ & \multicolumn{1}{l}{ P1 } & P2 & MSE & $\mathrm{R}^{2}$ \\
\hline ACTEFF & IBMEFF & 0.1366 & 1.1500 & 863.66 & 0.8913 \\
ACTEFF & SLIMEFF & 0.5436 & 1.0219 & 944.72 & 0.8811 \\
ACTEFF & COCOMOEFF & 1.5027 & 1.1095 & 1691.76 & 0.7870 \\
ACTEFF & ADJUSTEFF & 0.4687 & 1.1464 & 723.28 & 0.9090 \\
ACTEFF & HLSTDEFF & 2.3811 & 0.8452 & 726.82 & 0.9085 \\
ACTEFF & V(G) & 0.5356 & 1.0819 & 771.88 & 0.9029 \\
ACTEFF & V & 0.1703 & 1.0579 & 1691.50 & 0.7871 \\
n & DSI & 1.0700 & 0.8715 & 3882.58 & 0.9175 \\
V & DSI & 8.3025 & 1.2530 & 14141900.00 & 0.9877 \\
V(G) & DSI & 0.0769 & 1.0830 & 757.75 & 0.9052 \\
& & & & & \\
\hline
\end{tabular}

wher

P1,P2: parameters of the nonlinear function $x=P 1(X)^{P 2}$

MSE, $R^{2}$ : same as those described in Table 16 
Table 18. Linear Regression Results of 324 NCR Programs

\begin{tabular}{|c|c|c|c|c|c|c|c|c|c|}
\hline \multirow{2}{*}{$\begin{array}{l}\text { DEPENDENT } \\
\text { VARIABLE }\end{array}$} & \multirow{2}{*}{$\begin{array}{l}\text { INDEPENDENT } \\
\text { VARIABLE }\end{array}$} & \multirow[b]{2}{*}{$\mathrm{R}^{2}$} & \multirow[b]{2}{*}{$\mathbf{r}$} & \multirow[b]{2}{*}{ MSE } & F-STAT & \multirow{2}{*}{$\mathrm{B}_{0}$} & \multirow{2}{*}{$\bar{B}_{1}$} & \multicolumn{2}{|l|}{ AVG SQRD } \\
\hline & & & & & $(0.001 \mathrm{sIG})$ & & & DEL RESIDUAL & $\mathrm{R}^{2} \mathrm{pr} \in \mathrm{d}$ \\
\hline$n$ & DSI & 0.8166 & 0.9037 & 8292.09 & 1433.74 & 84.4777 & 0.4240 & 8713.81 & 0.81 \\
\hline$v$ & DSI & 0.9788 & 0.9893 & 10332555.80 & 14862.50 & -1585.2900 & 48.1909 & 10757778.27 & 0.98 \\
\hline$V(G)$ & DSI & 0.8760 & 0.9359 & 448.72 & 2274.41 & -2.4229 & 0.1241 & 456.43 & 0.87 \\
\hline
\end{tabular}

where

$\mathrm{R}^{2}, \mathrm{r}, \mathrm{MSE}, \mathrm{F}-\mathrm{STAT}, \bar{B}_{0}, \bar{B}_{1}$, AVG SQRD DEL RESIDUAL, Rpred, $\mathrm{n}, \mathrm{V}, \mathrm{V}(\mathrm{G})$, and DSI are the same as those described in Table 16 
Table 19. Nonlinear Regression Results of 324 NCR Programs

\begin{tabular}{|c|c|c|c|c|c|}
\hline $\begin{array}{l}\text { DEPENDENT } \\
\text { VARIABLE }\end{array}$ & $\begin{array}{c}\text { INDEPENDEI } \\
\text { VARIABLE }\end{array}$ & $P 1$ & P2 & MSE & $\mathrm{R}^{2}$ \\
\hline n & DSI & 5.1346 & 0.6744 & 6312.73 & 0.8604 \\
\hline v & DSI & 28.2923 & 1.0694 & 10137100.00 & 0.9792 \\
\hline$V(G)$ & DSI & 0.0816 & 1.0560 & 444.74 & 0.8771 \\
\hline
\end{tabular}

where

P1, P2 and $R^{2}$ are the same as those described in Table 17. 


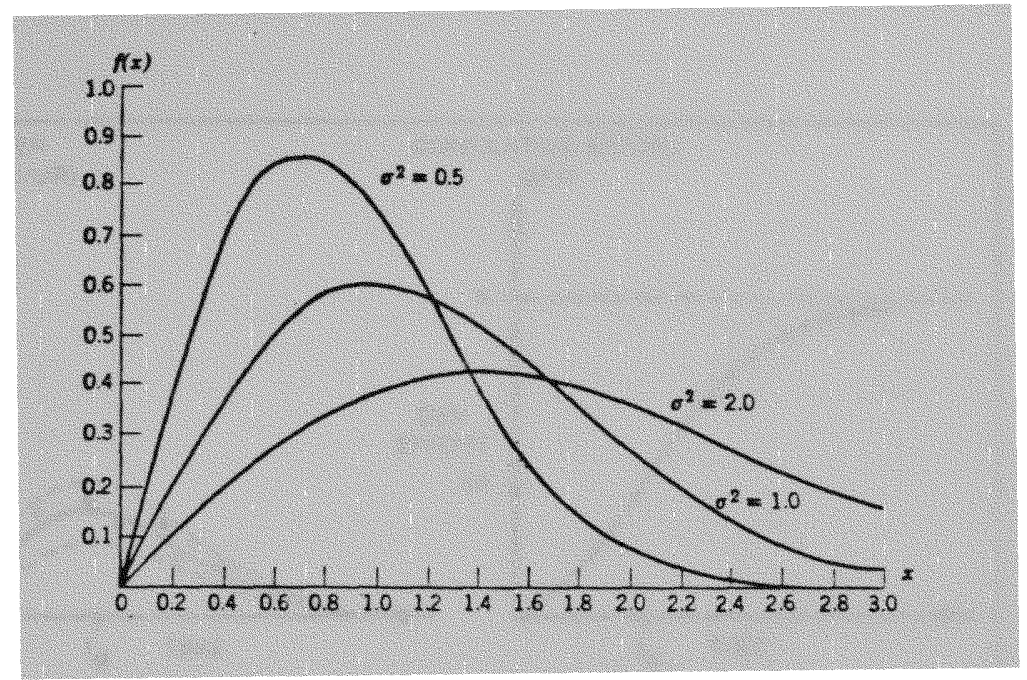

Figure 1. Rayleigh Distributions with Various values of Variance

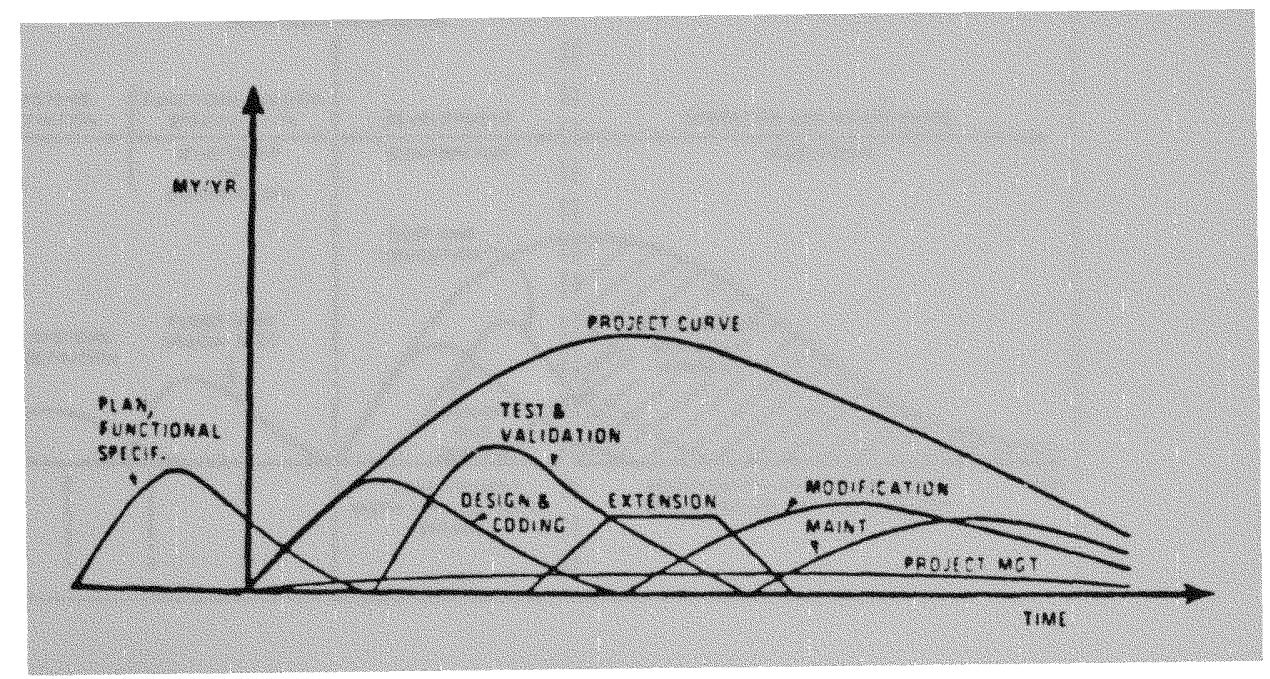

Figure 2. Pattern of Life-Cycle Effort Required to Complete a Large scale Software Project 


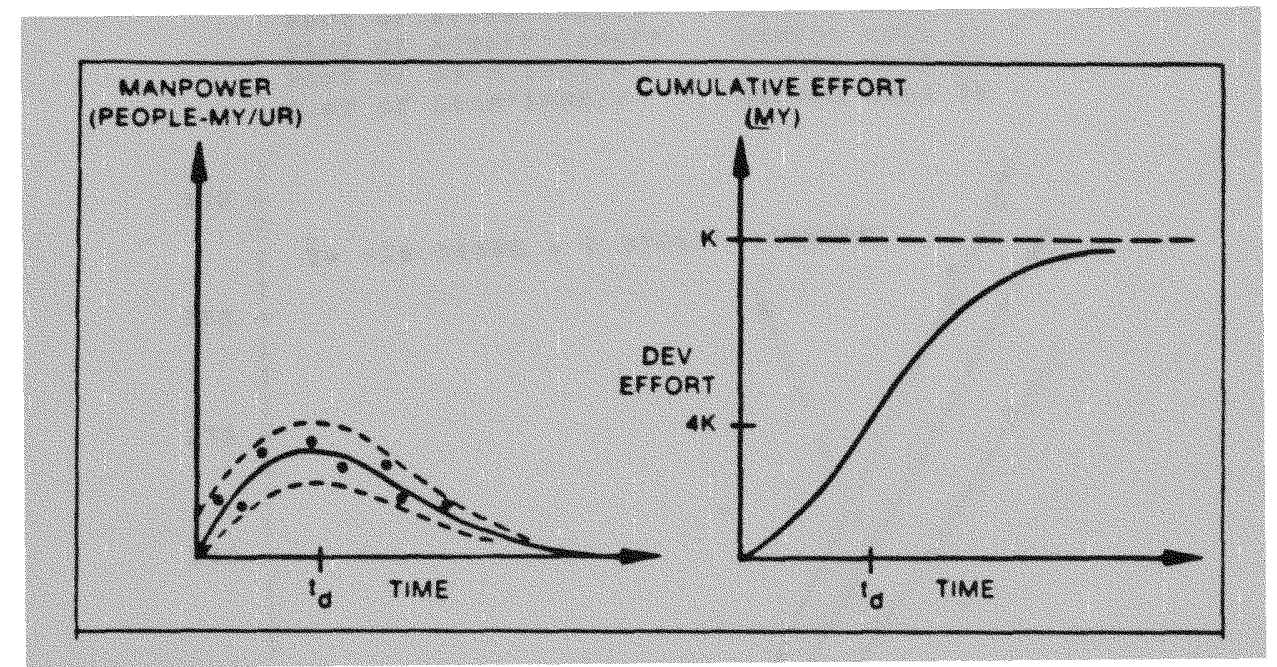

Figure 3. Manpower Pattern of Software Development for Large Software systems Interpreted by Putnam

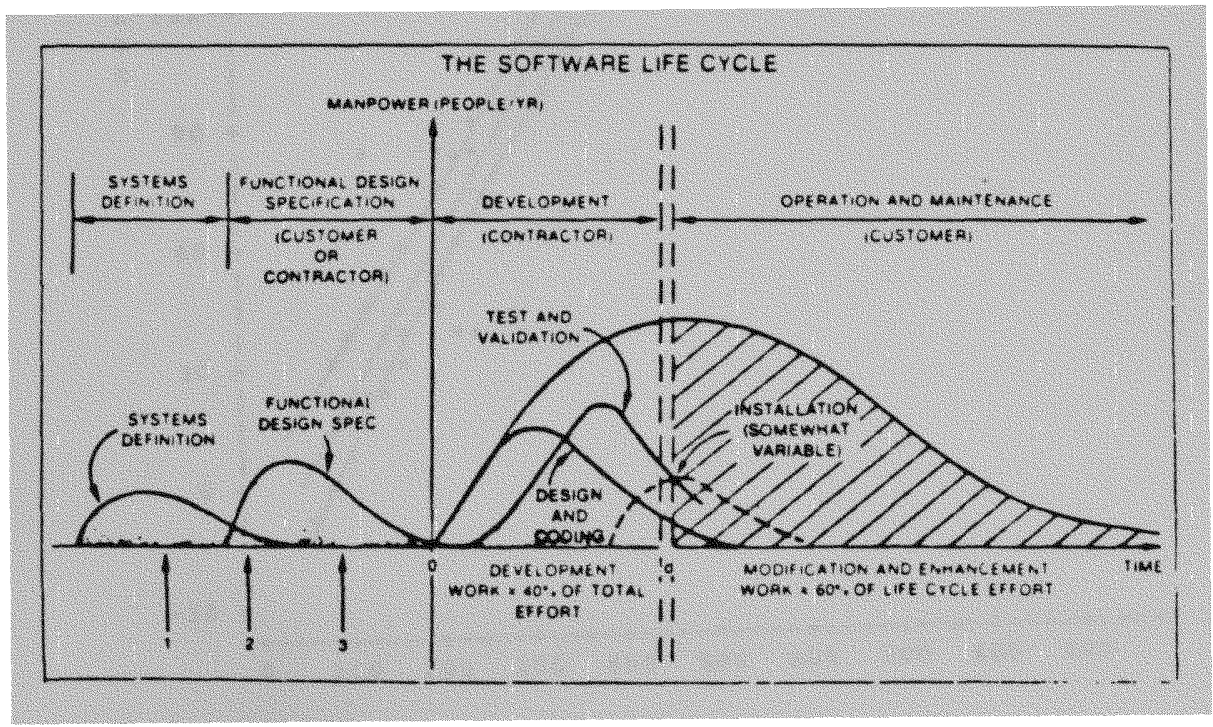

Figure 4. Software Life Cycle with stages of software Development Interpreted by Putnam 


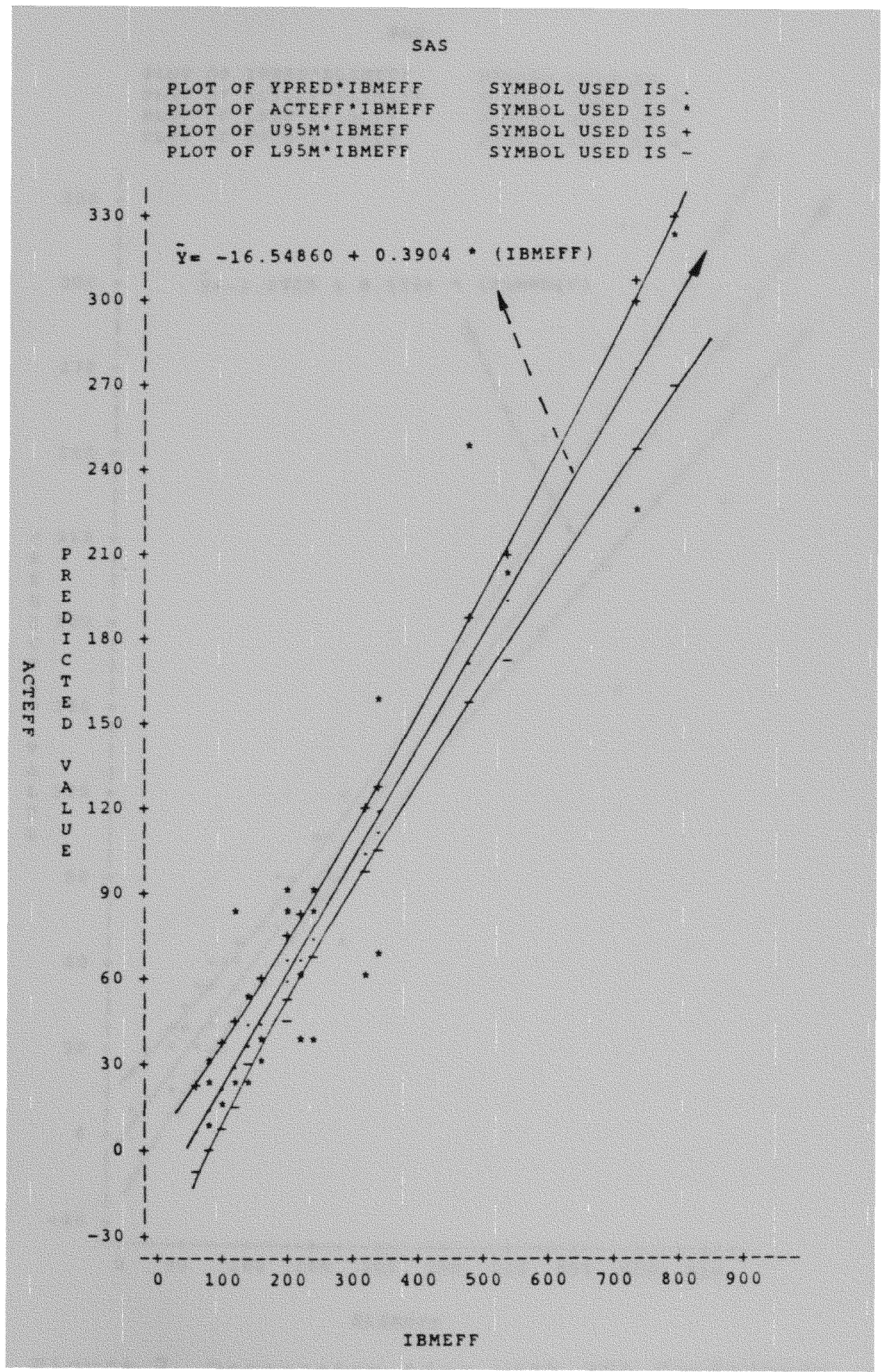

Figure 6. Regression Line with $95^{\circ}$ Confidence Interval for ACTEFF on IBMEFF 


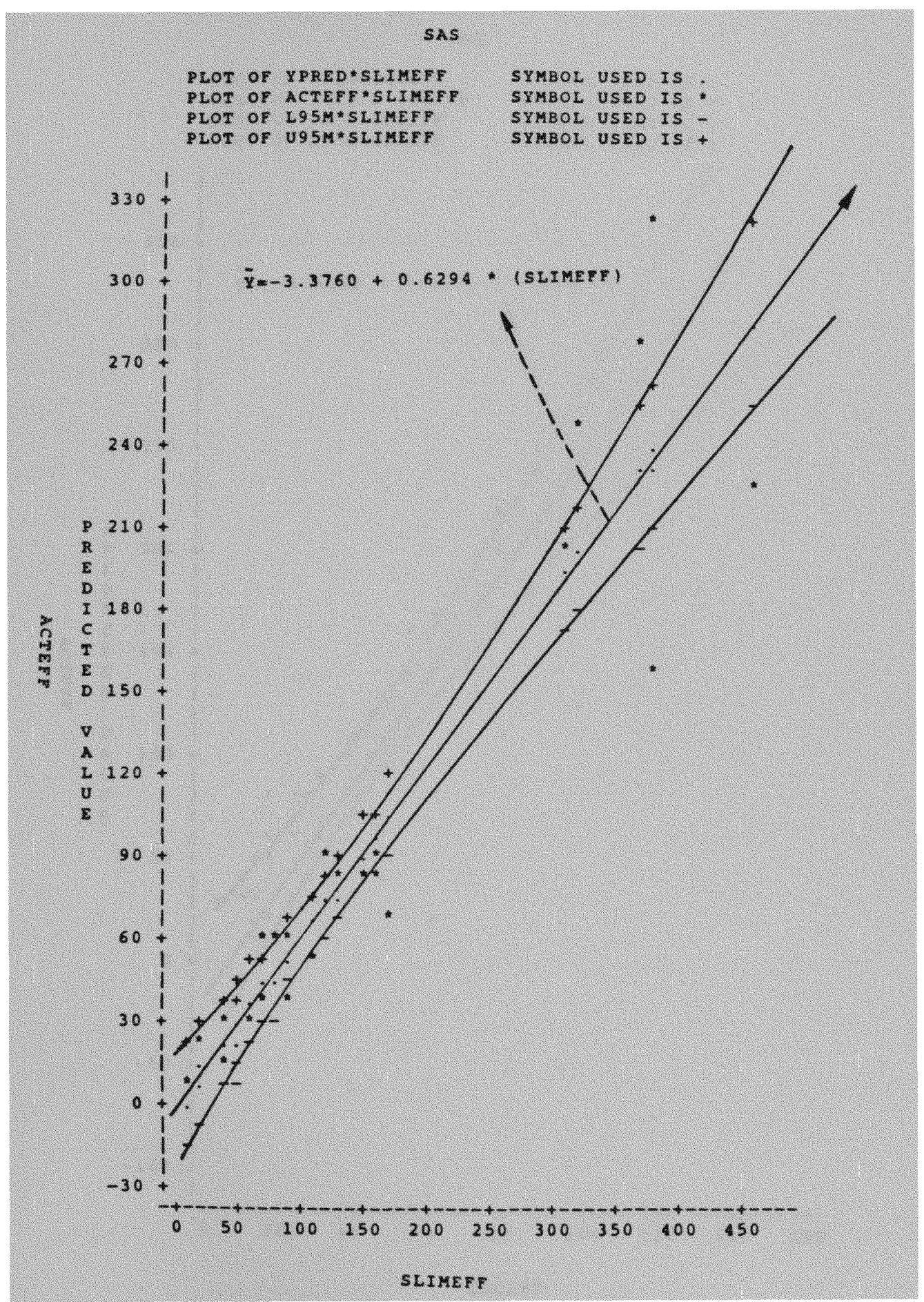

Figure 7. Regression Line with 958 Confidence Interval for ACTEFF on SLIMEFF 


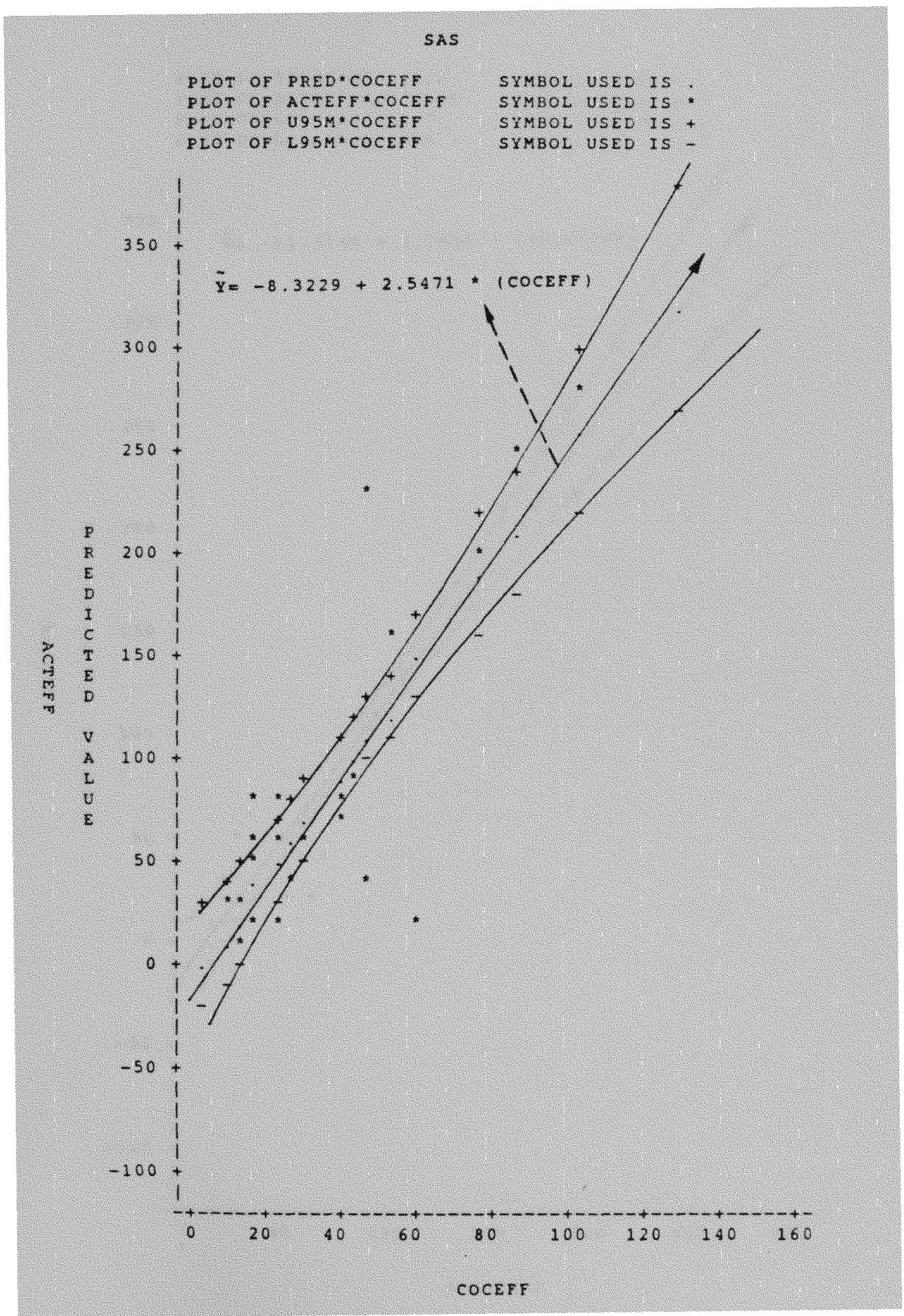

Figure 8 . Regression Line with $95 \%$ Confidence Interval for ACTEFF on COCOMOEFF 


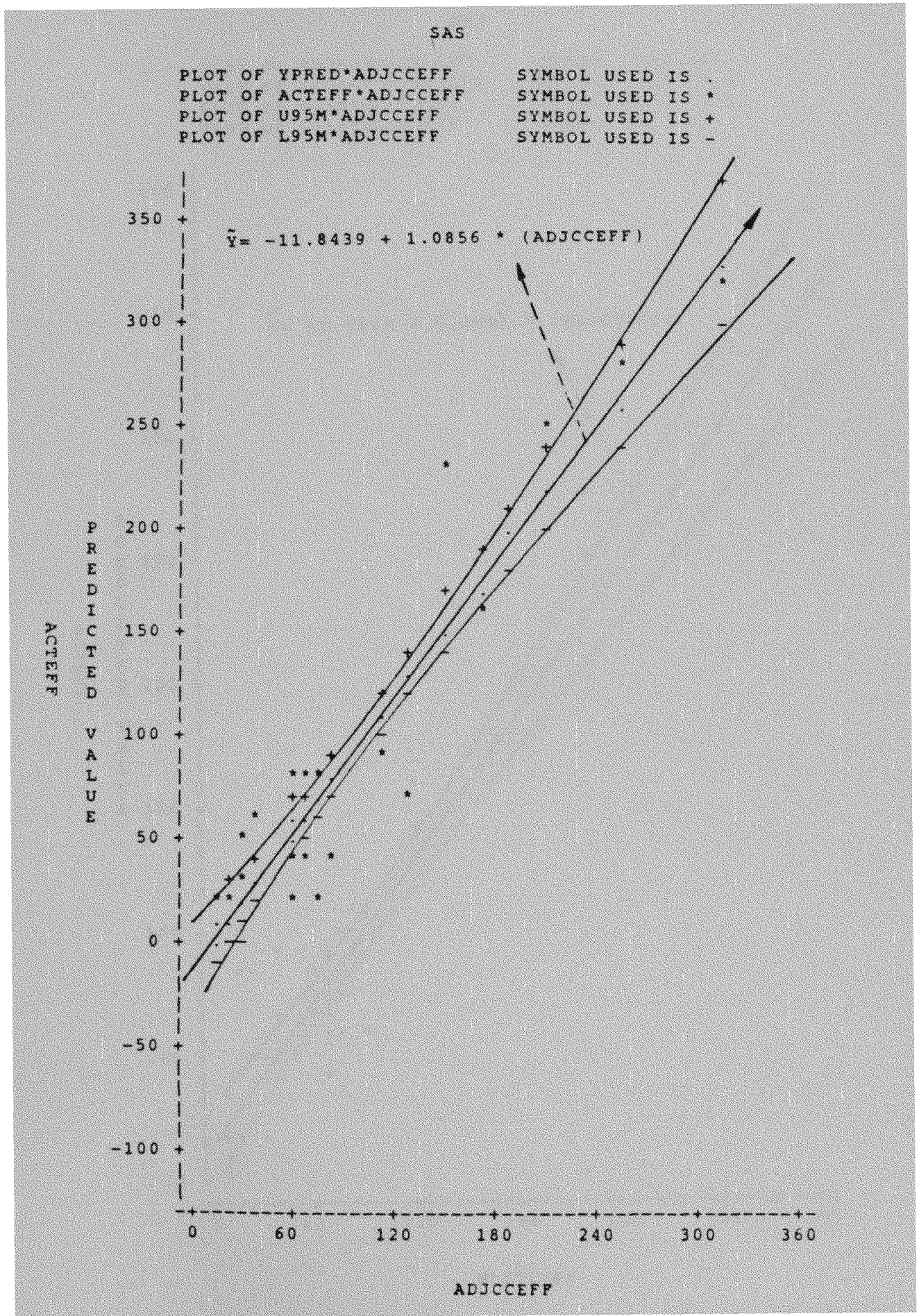

Figure 9. Regression Line with $95 \%$ Confidence Interval for ACTEFF on ADJUSTEFF 


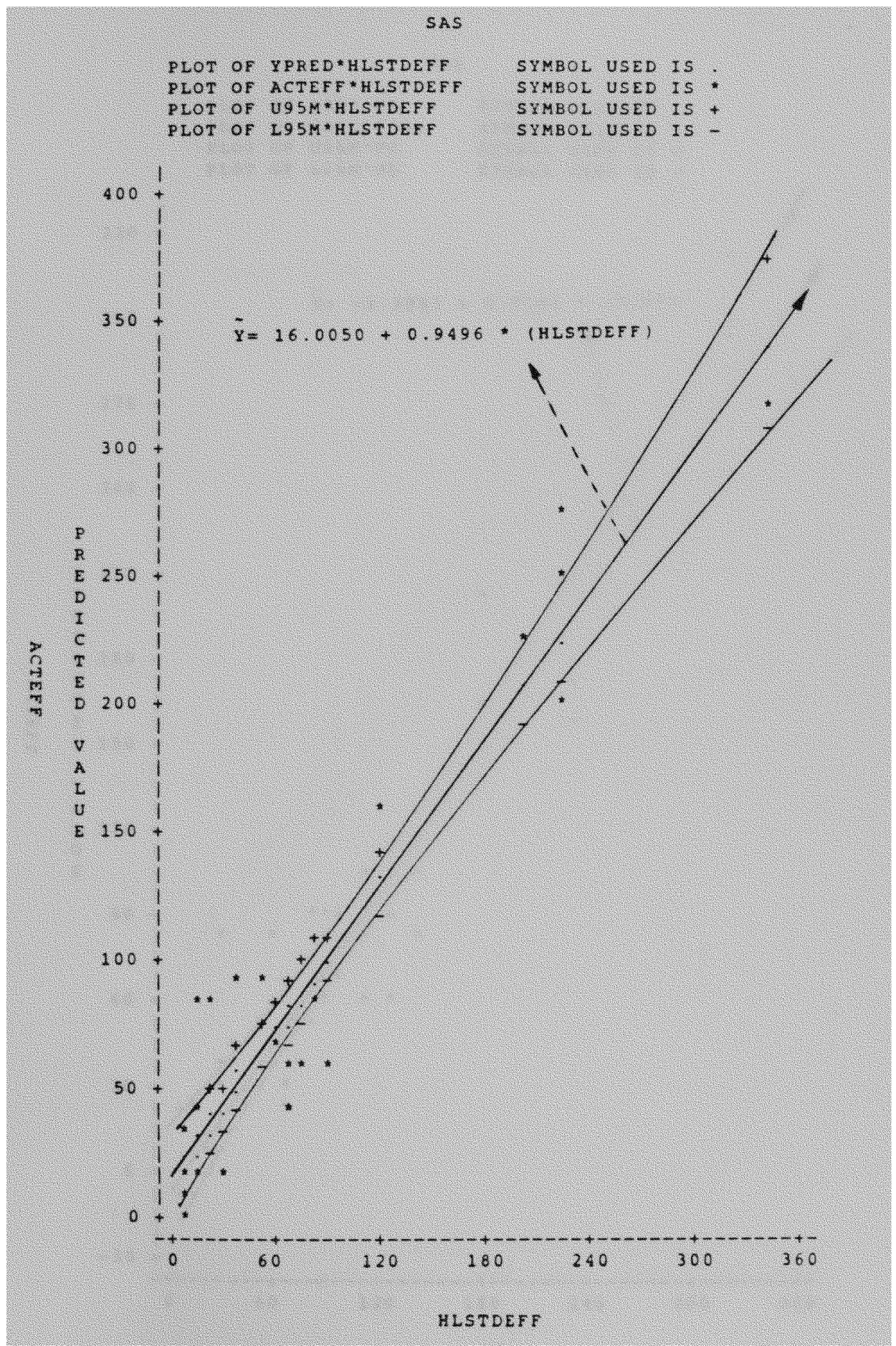

Figure 10. Regression Line with $95^{\circ}$ Confidence Interval for ACTEFF on HLSTDEFF 


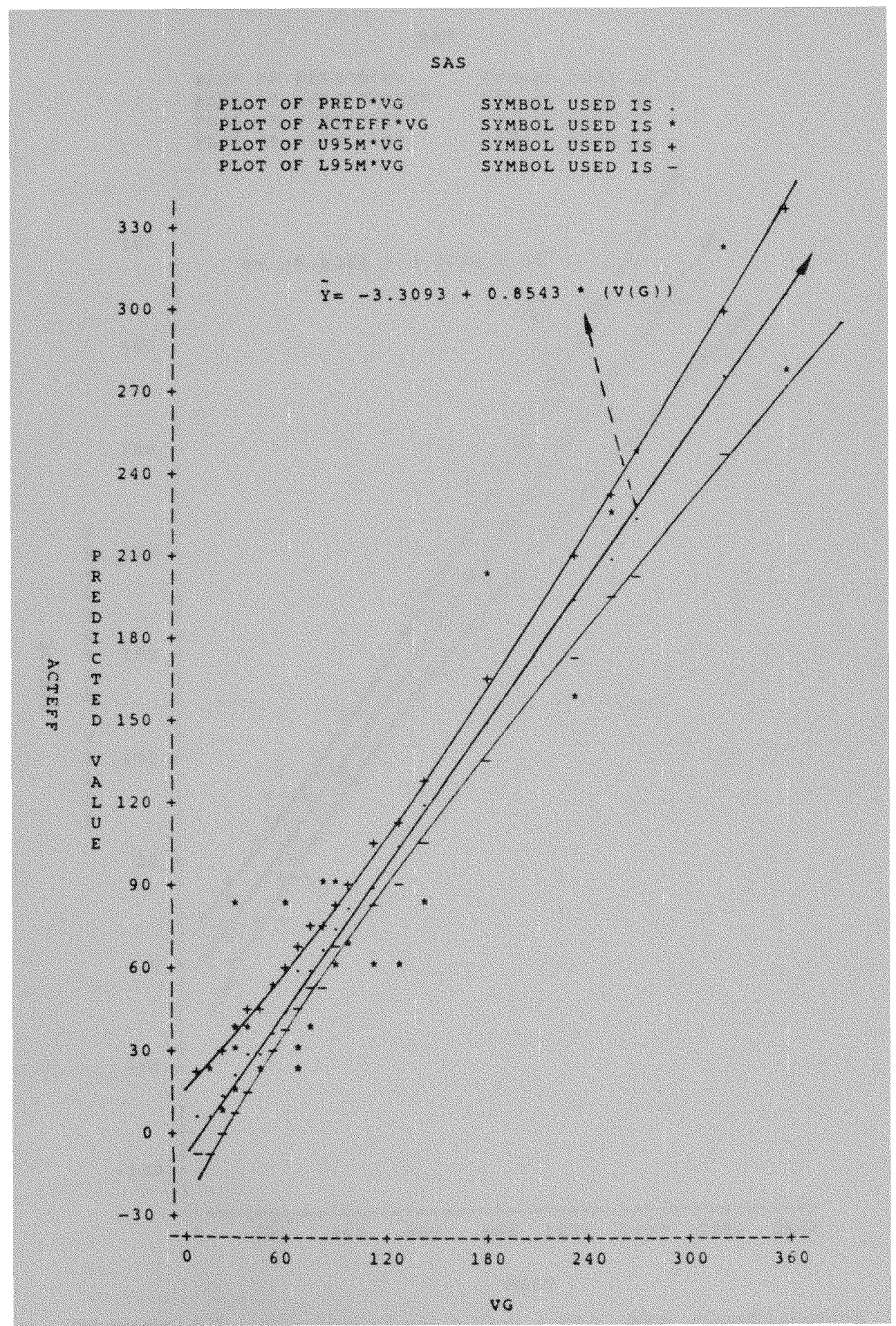

Figure 11. Regression Line with $95 \%$ Confidence Interval for ACTEFF on $V(G)$ 


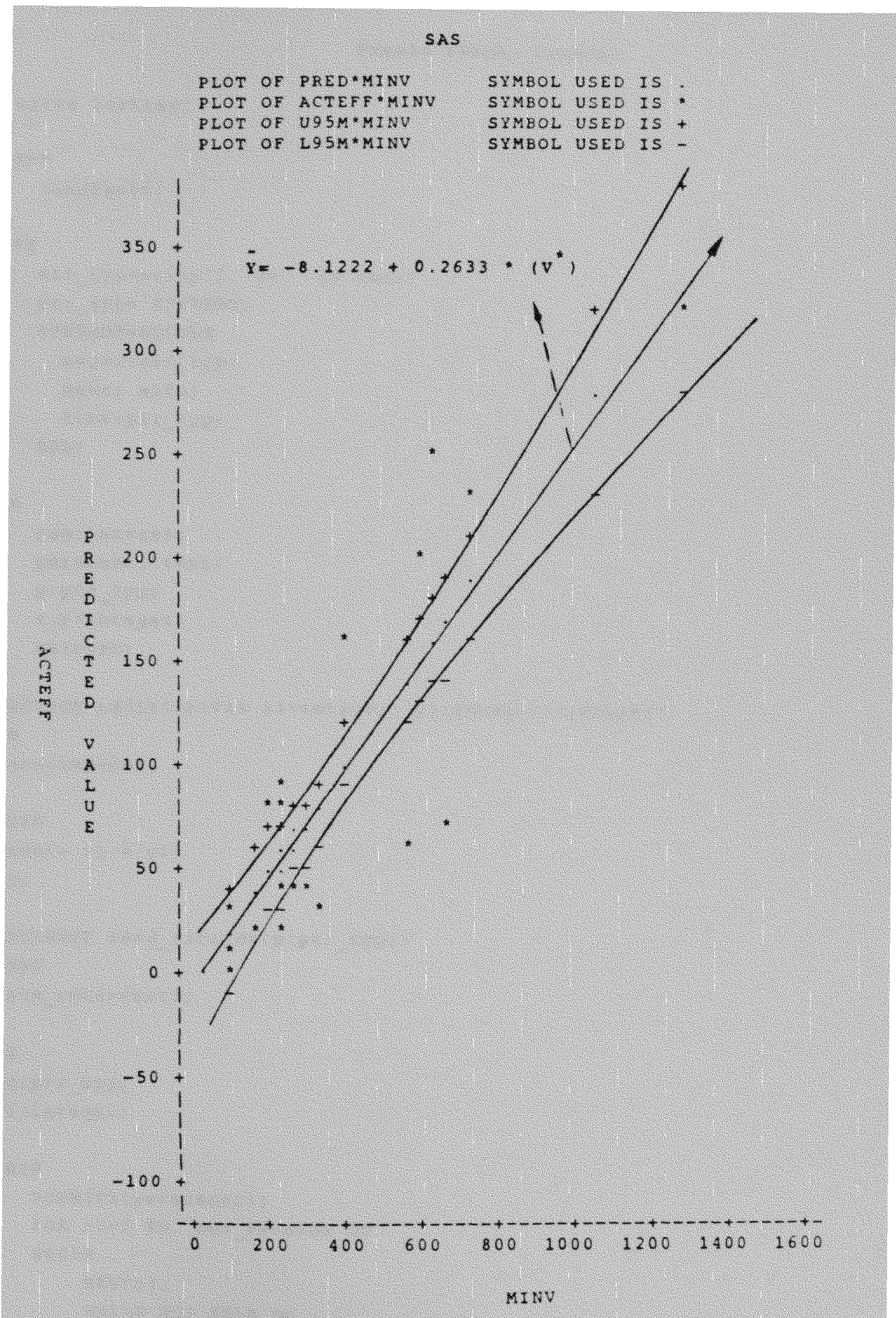

Figure 12. Regression Line with $95 \%$ Confidence Interval for ACTEFF on $\mathrm{V}$ 
APPENDIX A

sample PASCAL Program

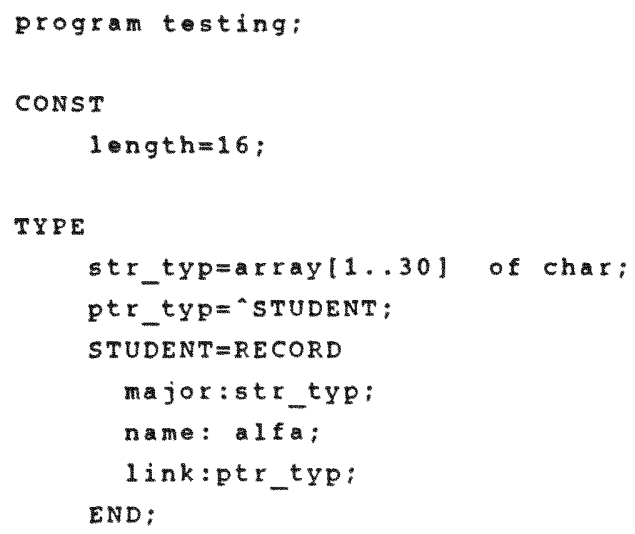


write(personn 1,p);

END; ( for )

END; (* proc*)

(* $\operatorname{main} *)$

BEGIN

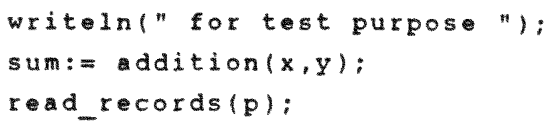

END. 


\section{Output of the Metrics Anlyzer Program}

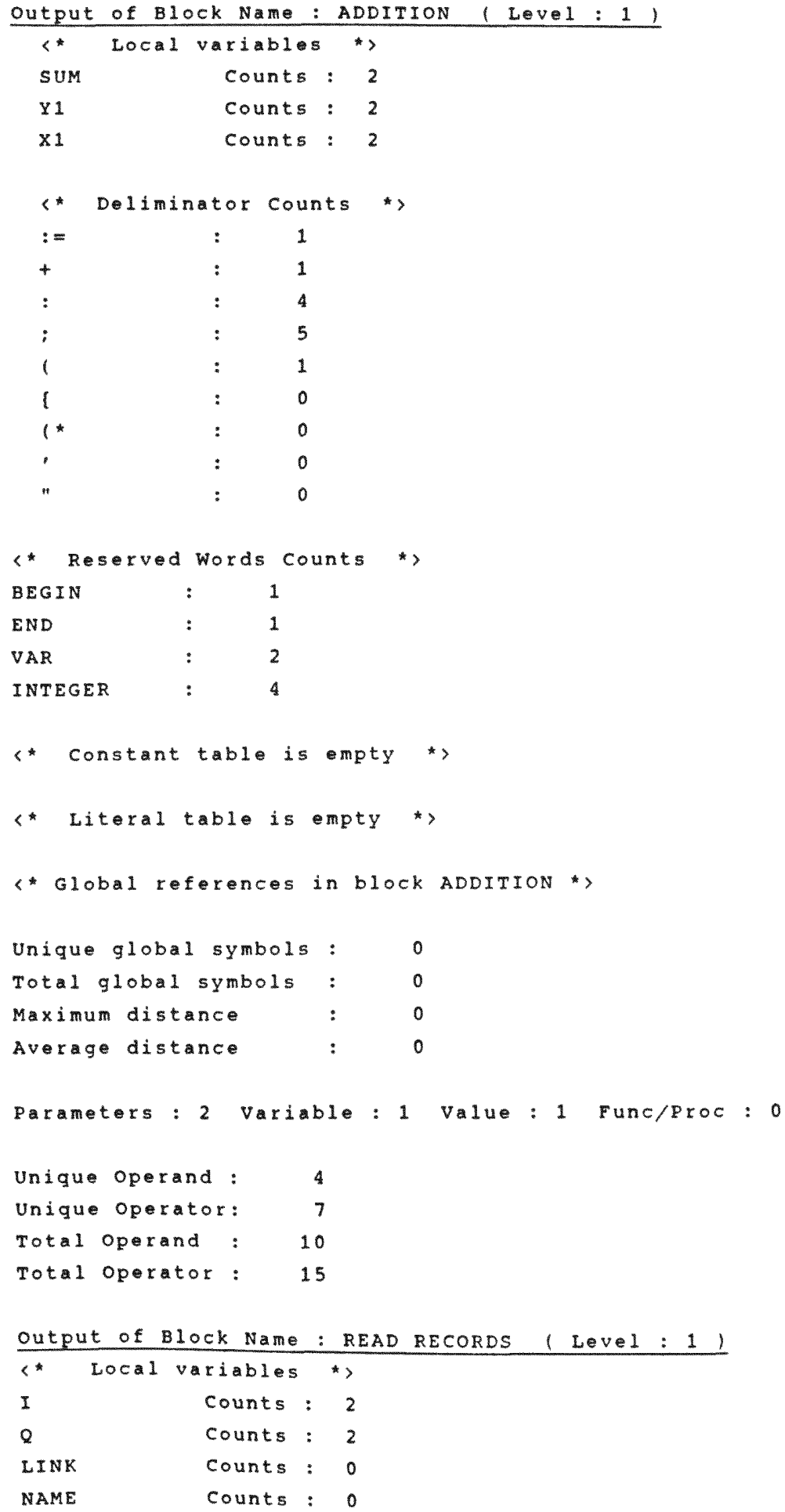




$\begin{array}{ll}\text { MAJOR } & \text { counts : } 0 \\ \text { NUM_RECORDS } & \text { counts : } 2 \\ \text { LINK } & \text { counts : } 7 \\ \text { NAME } & \text { counts : } 1 \\ \text { MAJOR } & \text { counts: } 1 \\ & \text { counts: } 1\end{array}$

$\begin{array}{ccc}\text { * Deliminator } & \text { Counts * } \\ := & : & 4 \\ = & : & 1 \\ : & : & 3 \\ : & : & 14 \\ , & : & 1 \\ : & : & 3 \\ 1 & : & 5 \\ 1 & : & 3 \\ \text { (* } & : & 0 \\ , & : & 2 \\ " & : & 1\end{array}$

(* Reserved Words counts *)

BEGIN : 3

CONST : 1

DO : 2

END : 3

FOR : 1

NIL : 1

NOT : 1

TO : 1

VAR : 1

WHILE : 1

INTEGER :

EOLN : 1

NEW : 1

READ : 1

REWRITE : 1

WRITE : 1

* Constant Table *

11

10

* Literal Table *

COMPUTER SCI 1

* Global references in block READ RECORDS *

PERSONNEL IVI: 1 cnt: 2

PTR_TYP lv1: 1 cnt: 2

unique global symbols : 2

Total global symbols : 4 


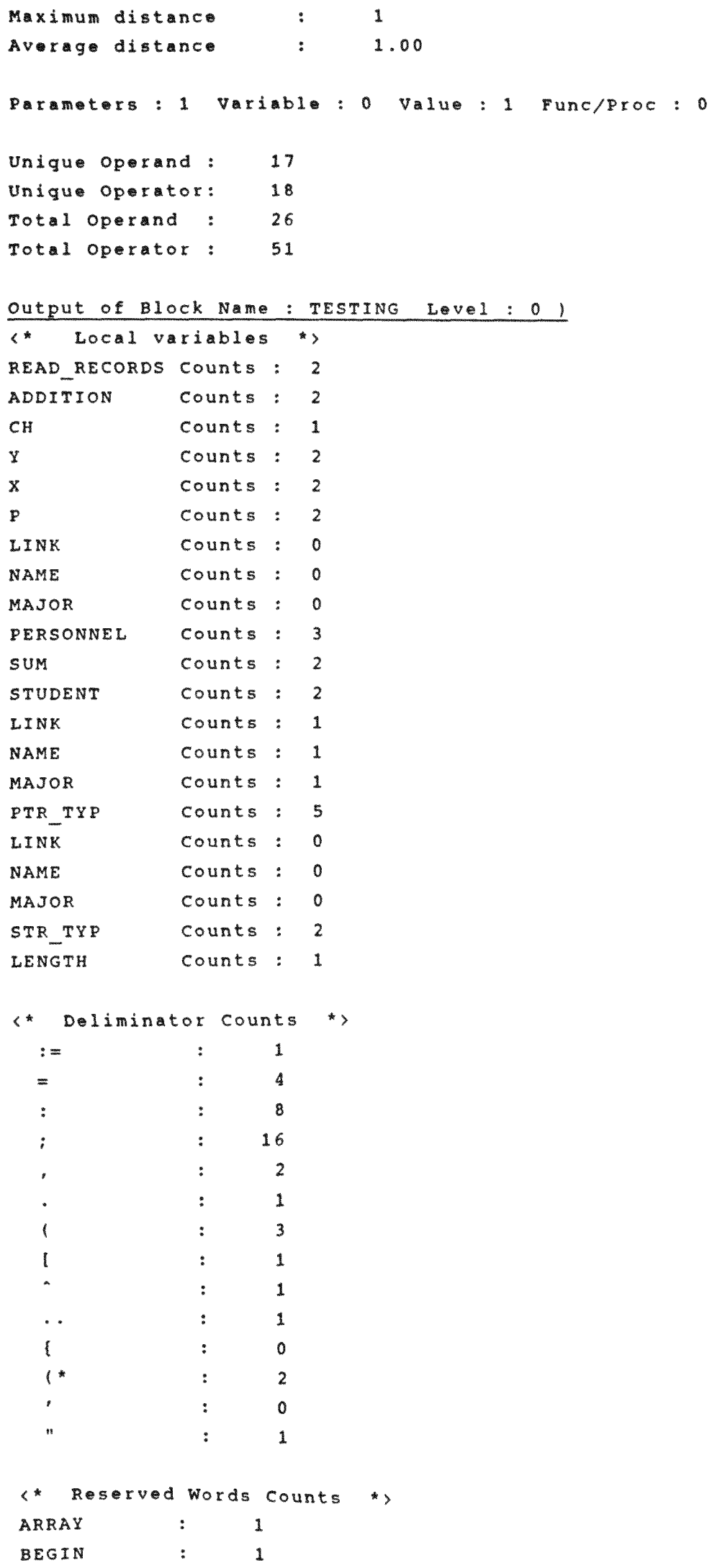




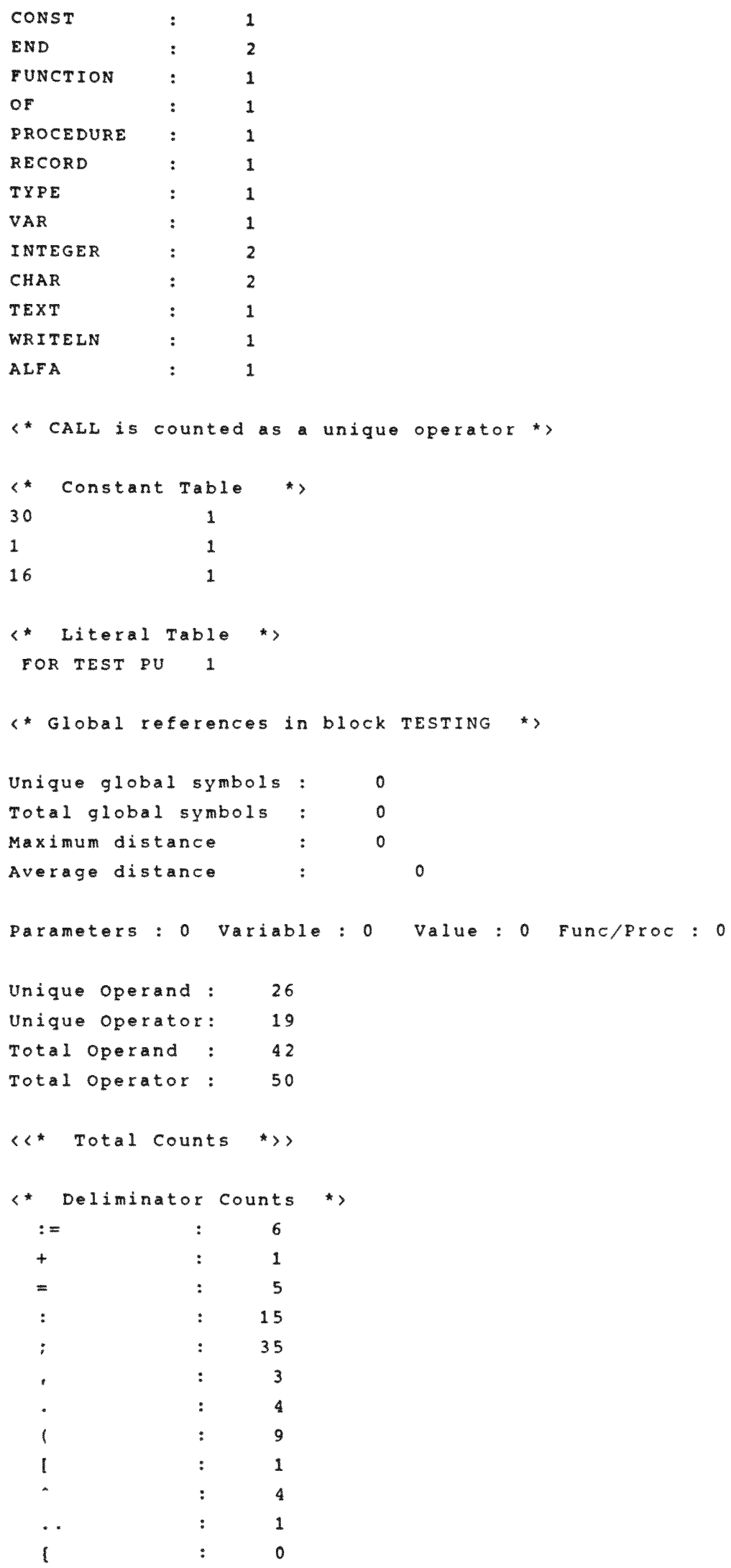




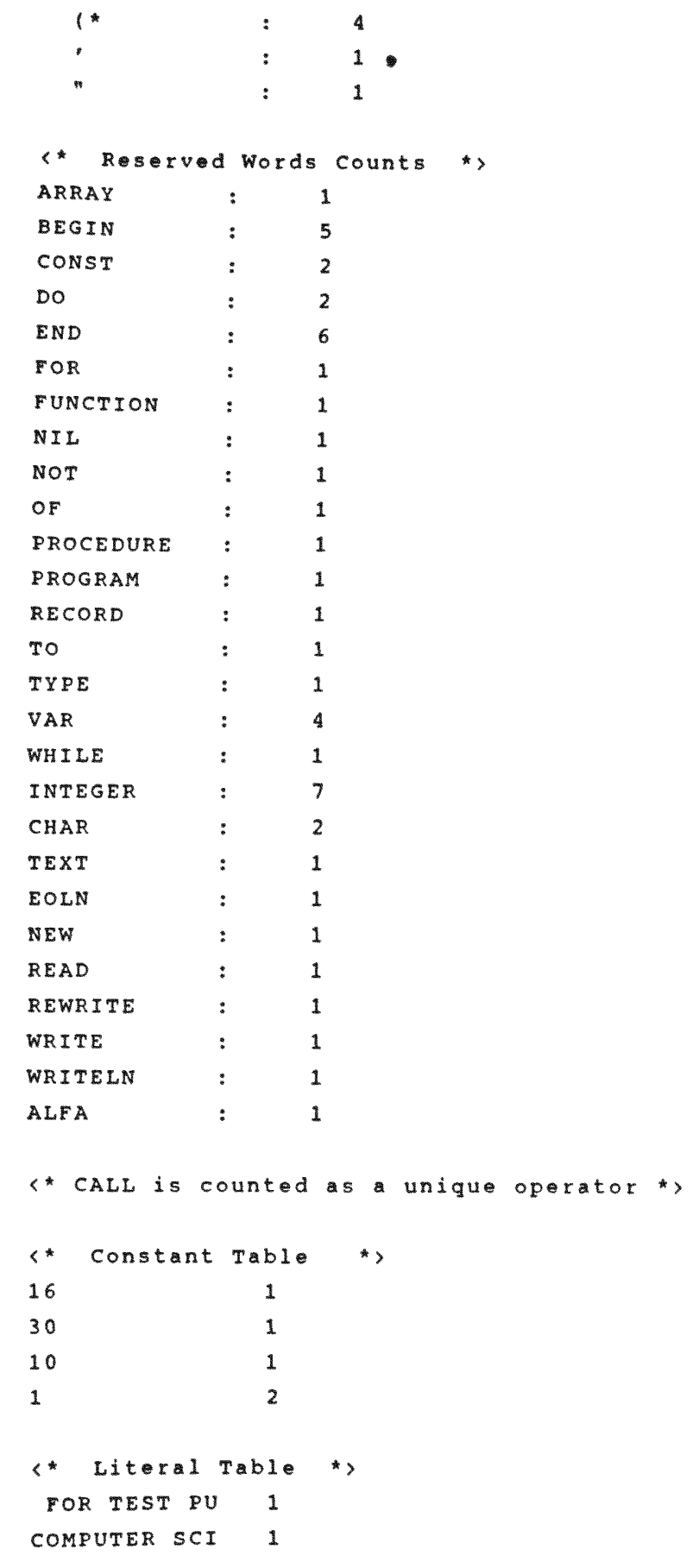

\begin{tabular}{llc}
\hline Total operators & $:$ & 117 \\
Total operands & $:$ & 79 \\
Total distinct identifiers & $:$ & 45 \\
Total distinct operators & $:$ & 26 \\
vocabulary size & $:$ & 71 \\
program length & $:$ & 196 \\
Expected program length & $:$ & 369.3448
\end{tabular}


program volume

Potential volume

Program level

Int ligence

Efort

Implementation time
1205.3504

52.8148

0.0438

52.8148

27508.7754

1528.2653 


\section{REFERENCES}

1. Albrecht, Allan J. and Gaffney John E. Jr., "Software Function, Source Lines of Code, and Development Effort Prediction: A Software science Validation," IEEE Transactions on Software Engineering, Vol. SE-9, No.6, November 1983 .

2. Boehm, Barry W., "Software and its Impact: Quantitative Assessment," Datamation, pp.48-59, May 1973.

3. Boehm, Barry W., "Software Engineering Ecomonics," Prentice-Hall, Inc., Englewood Cliffs, N.J., 1981.

4. Boehm, Barry W., "Software Engineering Economics," IEEE Trans. Software Eng., vol. SE-10, No.1, pp.4-19, January 1984.

5. "BMDP Biomedical Computer Programs P-Series", University of California press, 1977.

6. Demarco, Tom, "Controlling Software Projects Management, Measurement \& Estimation," Yourdon Press, New York, 1982 .

7. Farely, Richard E., "Software Engineering Concepts," MCGraw- Hill, New York, 1985.

8. Freiman, Frank R. and Park Robert E., "PRICE Software Model-Version 3: An Overiew," Proceedings, IEEE-PINY Workshop on Quantitative Software Models, Catalog No. TH0067-9, pp.32-39, October 1979.

9. Halstead, Maurice H. "Elements of Software Science," Elsevier North-Holland, Inc., New York, 1977.

10. Henry, F. Dircks, "Gruman's Software Cost Estimating Model," The proceedings for the National Aerospace and Electronics Conference, pp.674-683, May 19-21, 1981. 
11. Henry S., and Kafura D., "Software structure Metrics Based on Information Flow," IEEE Trans. on Software Engineering, Vol. SE-7, No.5, pp.510-518, Sept. 1981.

12. Jensen, Randall W., "An Improved Macrolevel Software Development Resource Estimation Model," Proceedings 5 th ISPA Conference, pp.88-92, April 1983.

13. Jensen, Randall $w .$, "Sensitivity Analysis of the Jensen Software Model," Proceedings 5th ISPA Conference, pp.384-389, April 1983.

14. McCabe, Thomas J. "A Complexity Measure," IEEE Transactions on Software Engineering, Vo1. SE-2, No.4, December 1976.

15. Meyer, Stuart L., "Data analysis for scientists and engineers," Jonh Wiley \& Sons, Inc., New York, 1975.

16. Montgomery, Douglas C., and Elizabeth A. Peck, "Introduction to Linear Regression Analysis," John Wiley \& Sons, Inc., New York 1982 .

17. Navlakha Jainendra K., and Qiang Li," Development of Software Interface Metrics", Phase I Report submitted to NCR, April 1985.

18. Ott, Lyman, "An Introduction to Statistical Methods and Data Analysis," PWS Publishers, Boston, Massachusetts 1984.

19. Putnam, Lawrence H., "A General Empirical Solution to Macro Software Sizing and Estimating Problem," IEEE Trans. On Software Eng., pp.345-361, July 1978.

20. Putnam, Lawrence. H., "Example of an Early Sizing, Cost, and Schedule Estimate for an Application Software system," Proceedings COMPSAC, 1978.

21. Putnam, Lawrence. H. and Fitzsimmons, A., "Estimating Software Costs, "Datamation, pp.189-198, Sept. 1979; Continued in Datamation pp.171-178, oct. 1979 and pp.137-140 Nov. 1979. 
22. Putnam, Lawrence H., "Tutorial softare cost Estimating and Life-Cycle control," IEEE Catalog NO. Eho 165-1, Library of Congress no. $80-83083,1980$.

23. "PRICE Software Mode1: Supplemental Information," RCA, Cherry Hill, N.J. March 1978.

24. "SAS User's Guide: Statistics", SAS Institute Inc., North Carolina, 1985.

25. Shapiro, Samuel S. \& Gerald J. Hahn, "Statistical Models $\frac{\text { in Engineering," John Wiley \& SOns, Inc., New York }}{1968 \text {. }}$

26. Walston C.E. and Felix C.P., "A Method of Programming Measurement and Estimation," IBM Systems J." pp.54-73 June 1977 . 27. Wang Institute, "WICOMO User's Manual,"
June 1984.

28. Wolverton, R.W., "The Cost of Developing Large Scale Software," TRW Inc., IEEE Tran. on Computer, VOL. C-23, No. 6, June 1974.

29. Yin B.H. and Winchester J.W., "The Establishment and Use of Measures to Evaluate the Quality of Software Designs, " Proceedings of the software and assurance workshop, pp. 45-52, Nov. 1978 . 